NISTIR 5009

\title{
A BIBLIOGRAPHY OF THE \\ NIST ELECTROMAGNETIC FIELDS \\ DIVISION PUBLICATIONS
}

Edited by

Ruth Marie Lyons

Kathryn A. Gibson 



\section{A BIBLIOGRAPHY OF THE \\ NIST ELECTROMAGNETIC FIELDS \\ DIVISION PUBLICATIONS}

\section{Edited by}

Ruth Marie Lyons

Kathryn A. Gibson

Electromagnetic Fields Division

Electronics and Electrical Engineering Laboratory

National Institute of Standards and Technology

Boulder, Colorado 80303-3328

September 1993

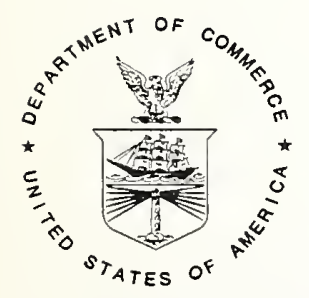

U.S. DEPARTMENT OF COMMERCE, Ronald H. Brown, Secretary

TECHNOLOGY ADMINISTRATION, Mary L. Good, Under Secretary for Technology

NATIONAL INSTITUTE OF STANDARDS AND TECHNOLOGY, Arati Prabhakar, Director 

CONTENTS

$\underline{\text { Page }}$

INTRODUCTION . . . . . . . . . . . . . . . . . . . . . . . . . . .

ANTENNAS . . . . . . . . . . . . . . . . . . . . . 3

DIELECTRIC MEASUREMENTS. . . . . . . . . . . . . . . . . . . . . 22

ELECTROMAGNETIC INTERFERENCE . . . . . . . . . . . . . . . . . 27

MICROWAVE METROLOGY. . . . . . . . . . . . . . . . . . . . 59

NOISE. . . . . . . . . . . . . . . . . . 81

REMOTE SENSING . . . . . . . . . . . . . . . . . . . 87

TIME DOMAIN. . . . . . . . . . . . . . . . . . . . . . . . . . .

WAVEFORM METROLOGY . . . . . . . . . . . . . . . . . . 92

MISCELLANEOUS. . . . . . . . . . . . . . . . . . . . . 100

AUTHOR INDEX . . . . . . . . . . . . . . . . . . . . . . . 110 



\title{
A BIBLIOGRAPHY OF THE NIST ELECTROMAGNETIC FIELDS DIVISION PUBLICATIONS
}

\author{
Edited by
}

Ruth Marie Lyons and Kathryn A. Gibson

This bibliography lists the publications by the staff of the Electromagnetic Fields Division of the National Institute of Standards and Technology for the period January 1970 through July 1993. It supersedes NISTIR 3993 which listed the publications of the Electromagnetic Fields Division from January 1970 through July 1992. Selected earlier publications from the Division's predecessor organizations are included.

Key words: antennas; dielectric measurements; electromagnetic interference; microwave metrology; microwave power, impedance, attenuation; near-field antenna measurements; noise; nonionizing radiation; radiation hazards; standards; time domain; waveform metrology.

\section{INTRODUCTION}

The Electromagnetic Fields Division was formed during the reorganization of NIST (formerly NBS) in April 1978. Originally, the Division developed measurement methods and standards, and provided metrological support for antenna systems, noise measurement equipment, fast pulse waveforms, electromagnetic interference, electromagnetic environmental characterization equipment, electromagnetic emission and immunity (or susceptibility) testing equipment, and especially for fields near sources or in other complicated field conditions. In April 1985, microwave and millimeter wave metrology was added to the Division.

We have attempted to include all work published by the Division staff in the period from January 1970 through July 1993. Work performed by authors before joining this Division or work by authors who have left this Division has been included only when it had special significance. A miscellaneous category includes general publications. A few references are listed in more than one category where appropriate.

Several other information sources may be useful to the reader who is interested in NIST activities connected with electromagnetic metrology. A companion bibliography, NISTIR 5008, lists publications of the Electromagnetic Technology Division for the same period. Topics include metrology for laser systems, optical fibers, optical communication equipment, cryoelectronics, superconductors, and other unusual electrical engineering materials. Three additional bibliographies list publications of the former Electromagnetics Division: NBSIR 73-320 (July 1972-June 1973); NBSIR 74-395 (July 
1973-June 1974); NBSIR 75-818 (July 1974-June 1975). These were preceded by a series of unpublished reports (edited by H. M. Altschuler) that cover the period back to 1956. An excellent summary of the whole field of electromagnetic metrology as it stood in 1967 was published as a special issue of the Proceedings of the IEEE (volume 55, June 1967). Advances in the following decades were described in two other special issues of the same journal (volume 66, April 1978 and volume 74, January 1986).

A Note on Abbreviations

NOTE: On August 23, 1988, the National Bureau of Standards (NBS) became the National Institute of standards and Technology (NIST); therefore, documents with either prefix are considered NIST publications. Most readers are familias with the commonly used abbreviations for the names of the professional journals that appear in this bibliography. Some publication series are peculiar to NIST and may call for explanation. They are:

NISTIR - NIST Interagency/Internal Report NIST Tech. Note - NIST Technical Note NIST SP - NIST Special Publication NIST HB - NIST Handbook NIST JRES - NIST Journal of Research NIST MN - NIST Monograph
NBSIR - NBS Interagency/Internal Report NBS Tech. Note - NBS Technical Note NBS SP - NBS Special Publication NBS HB - NBS Handbook NBS JRES - NBS Journal of Research NBS MN - NBS Monograph

Purchase Procedures and Document Availability

NIST (NBS) Technical Notes, Special Publications, Handbooks, Journals of Research, and Monographs may be purchased from the U.S. Government Printing office at the following address: New Orders, Superintendent of Documents, P.O. Box 371954, Pittsburgh, PA 15250-7954. Orders may be payed by major credit card, NTIS Deposit Account, or check or money order payable in U.S. dollars to the superintendent of Documents. The Government Printing office usually only stocks these publications for a year or two, after which they may be purchased from the National Technical Information Service at the address listed below.

NIST (NBS) Interagency/Internal Reports (NISTIRs, NBSIRS) may be purchased from the National Technical Information Service, Springfield, VA 22161, (703) 487-4650. orders may be payed by major credit card, NTIS Deposit Account, or check or money order payable in U.S. dollars to NTIS.

Reprints of papers published in non-NIST media may be available in limited quantities from the authors. 


\section{ANTENNAS}

FRANCIS, M. H.; REPJAR, A. G.; KREMER, D. P.

A Certification Plan for a Planar Near-Field Range Used for HighPerformance Phased-Array Testing

Proc., Antenna Meas. Tech. Assoc. Symp., 19-23 October 1992, Columbus, $\mathrm{OH}$, pp. 8-24 through 8-30

October 1992

GUERRIERI, J.R.; TAMURA, D. T.; REPJAR, A. G.

Accurate Planar Near-Field Probe Correction Using Dual-Pirt CircularlyPolarlized Probes

Proc., Antenna Meas. Tech. Assoc. Symp., 19-23 October 1992,

Columbus, $\mathrm{OH}$, pp. 10-17 through 10-25

october 1992

MACREYNOLDS, R.; REPJAR, A. G.; KREMER, D. P ; CANALES, S .

Phased Array Testing and Diagnostics Using Planar Near-Field Scanning Proc., Antenna Meas. Tech. Assoc. Symp., 19-23 October 1992, Columbus, $\mathrm{OH}, \mathrm{pp}$. 8-8 through 8-13 october 1992

WITTMANN, R. C.; NEWELL, A. C.; STUBENRAUCH, C. F.; MACREYNOLDS, R.; FRANCIS, M. $\mathbf{H}$.

Simulation of the Merged-Spectrum Techniques for Aligning Planar PhasedArray Antennas, Part I NISTIR 3981

October 1992

FRANCIS, M. H.; REPJAR, A. G.; KREMER, D. P.

A Certification Plan for a Planar Near-Field Range Used for HighPerformance Phased-Array Testing NISTIR 3991

July 1992

MUTH, L. A.

Probe-Position Error Correction in Planar Near-Field Measurement at 60

$\mathrm{GHz}$ : Experimental Verification

NIST JRES, Vol. 97, No. 2, pp. 273-297

March-April 1992

WITTMANN, R. C.

Probe-Corrected Spherical Near-Field scanning Theory in Acoustics

IEEE Trans. Instrum. Meas., Vol. IM-41, No. 1, pp. 17-21

February 1992

FRANCIS, M. H.: WITTMANN, R. C.

Swept Frequency Gain Measurements from 33-50 GHz at the National

Institute of standards and Technology

Proc., 13th Annual Meeting and Symp., Ant. Meas. Tech. Assoc., 711 October 1991, Boulder, CO, pp. 1-3 through 1-6

October 1991

REPJAR, A. G.; GUERRIERI, J. R.; KREMER, D. P.; CANALES, S . Determining Faults on a Flat Phased Array Antenna Using Planar NearField Techniques

Proc., 13th Annual Meeting and Symp., Ant. Meas. Tech. Assoc., 711 October 1991, Boulder, CO, pp. 8-11 through 8-19

october 1991 
WITTMANN, R. C.; YAGHJIAN, A. D.

Spherical-Wave Expansions of Piston-Radiator Fields

J. Acoust. Soc. Amer., Vol. 30, No. 3, pp. 1647-1655

September 1991

MUTH, I. A.

General Analytic Correction for Probe-Position Errors in Spherical NearField Measurements

NIST JRES, Vol. 96 , No. 4, pp. 391-410

July-August 1991

MUTH, L. A.; LEWIS, R. L.

Personal Computer Codes for Analysis of Planar Near Fields

NISTIR 3970

June 1991

WITTMANN, R. C.

Probe-Corrected Spherical Near-Field Scanning Theory in Acoustics

Proc., IEEE Instrum./Meas. Technol. Conf., 14-16 May 1991,

Atlanta, GA, pp. 283-286

May 1991

ESTIN, A. J.; JANEZIC, M. D.

Improvements in Dielectric Measurements with a Resonant Cavity

Proc., IEE Instrum. Meas. Tech. Conf., 14-16 May 1991, Atlanta, GA, Pp. 573-579

May 1991

MUTH, L. A.

Analytic Correction for Probe-Position Errors in spherical Near-Field Measurements

Proc., 7th Intl. Conf. on Antennas and Propagat., 15-18 April

1991, York, England, pp. 762-765

April 1991

MUTH, L. A.; LEWIS, R. L.

A General Technique to Correct Probe Position Errors in Planar Near-

Field Measurements to Arbitrary Accuracy

IEEE Trans. Antennas Propagat., Vol. 38, No. 12, pp. 1925-1932

December 1990

FRANCIS, M. H : MACREYNOLDS, $\mathbf{R}$.

Evaluation of Dual-Port Circularly Polarized Probes for Planar Near-

Field Measurements

Proc., 12th Annual Meeting and Symp., Ant. Meas. Tech. Assoc., 8-

11 october 1990, Philadelphia, PA, pp. 13-3 through 13-8

October 1990

MUTH, L. A.; NEWELL, A. C.; IEWIS, R. L.; CANALES, S.; RREMER, D. P. Experimental and Theoretical Probe Position Error Correction in NearField Antenna Measurements

Proc., 12th Annual Meeting and Symp., Ant. Meas. Tech. Assoc., 8-

11 October 1990, Philadelphia, PA, Pp. 13-27 through 13-30 october 1990

KREMER, D. P.; NEWELI, A. C.

Alignment Fixture for Millimeter Waveguide

IEEE Trans. Antennas and Propagat., Vol. 32, No. 3, pp. 45-48 June 1990 
WITTMANN, R. C.

Spherical Near-Field Scanning: Determining the Incident Field Near a Rotatable Probe

Proc., IEEE Intl. Symp. Ant. Propagat. Soc., 7-11 May 1990,

Dallas, TX, pp. 224-227

May 1990

HILI, D. A.; EBRET, R. L.

Near-Field Gain of Pyramidal Horns from 18 to $40 \mathrm{GHz}$

NISTIR 89-3924

November 1989

FRANCIS, M. H.

Antenna Far-Field Pattern Accuracies at Millimeter Wave Frequencies

Using the Planar Near-Field Technique

Proc., Ant. Meas. Tech. Assoc. Symp., 9-13 october 1989, Monterey, $\mathrm{CA}$, pp. 11-16 through 11-21

October 1989

GUERRIERI, J.R.; KREMER, D. P.

Automated Multi-Axis Motor Controller and Data Acquisition System for Near-Field Scanners

Proc., Ant. Meas. Tech. Assoc. Symp., 9-13 October 1989, Monterey, CA, pp. 12-24 through 12-28

october 1989

KREMER, D. P.; NEWEIL, A. C.; AGEE, D. A.

Absorber Characterization

Proc., Ant. Meas. Tech. Assoc. Symp. 9-13 October 1989, Monterey, $\mathrm{CA}$, pp. 13-7 through 13-11.

October 1989

NEWELI, A. C.; RREMER, D. P.; GUERRIERI, J. R.

Improvements in Polarization Measurements of Circularly Polarized Antennas

Proc., Ant. Meas. Tech. Assoc. Symp., 9-13 October 1989, Monterey, $C A$, pp. 1-30 through 1-36

October 1989

NEWELL, A. C.; GUERRIERI, J. R.; PERSINGER, R. R.; STILES, J. A.; MCFARLANE, E. J .

Comparison of Antenna Boresight Measurements Between Near-Field and Far-Field Ranges

Proc., Ant. Meas. Tech. Assoc. Symp., 9-13 October 1989, Monterey, CA, pp. 1-24 through 1-29

october 1989

MUTH, L. A.; LEWIS, R. L.

Planar Near-Field Codes for Personal Computers NISTIR 89-3929 october 1989

An Iterative Technique to Correct Probe Position Errors in Planar Near-Field to Far-Field Transformations Proc., 1989 Intl. Symp. on Antennas and Propagat., 22-25 August 1989, Nagoya, Japan, pp. 901-904 August 1989

WITTMANN, R. C.

Comments on Spherical Near-Field Antenna Measurements Review for Ant. Propagat. Soc. Newsletter, Vol. 31, No. 3, pp. 35-36

June 1989 
MUTH, L. A.; LEWIS, R. L.

An Iterative Technique to Correct Probe Position Errors in Planar

Near-Field to Far-Field Transformations

NIST Tech. Note 1323

October 1988

FRANCIS, M. H.; REPJAR, A. G.; RREMER, D. P.

Antenna Measurements for Millimeter Waves at the National Bureau of Standards

Proc., Ant. Meas. Tech. Assoc., 12-16 september 1988, Atlanta, GA, pp. 13-13 through 13-17

September 1988

RREMER, D. P.; REPJAR, A. G .

Antenna Calibrating Standards Using $\mathbf{C W}$ and Pulsed-CW Measurements and the Planar Near-Field Method

Proc., Ant. Meas. Tech. Assoc., 12-16 September 1988, Atlanta, GA, pp. 13-21 through 13-29

September 1988

WITTMANN, R. C.

Spherical Wave Operators and the Translation Formulas

IEEE Trans. Antennas Propagat., Vol. 36, No. 8, pp. 1078-1087 August 1988

NEWELL, A. C.

Development of Near-Field Test procedures for Communication Satellite

Antennas, Phase I, Part 2

NBSIR 87-3081

August 1988

Improved Polarization Measurements Using a Modified Three Antenna Technique

IEEE Trans. Antennas Propagat., Spec. Issue on Near-Field Scanning

Techn., Vol. 36, No. 6, pp. 852-854 June 1988

Error Analysis Techniques for Planar Near-Field Measurements

IEEE Trans. Antennas Propagat., Spec. Issue on Near-Field scanning Techn., Vol. 36, No. 6, pp. 754-768

June 1988

BAIRD, R. C.; NEWEIL, A. C.; STUBENRAUCH, C. F.

A Brief History of Near-Field Measurements of Antennas at the National Bureau of Standards

IEEE Trans. Antennas Propagat., Spec. Issue on Near-Field Scanning Techn., Vol. 36, No. 6, pp. 727-733 June 1988

IEWIS, R. I.; NEWELI, A. C.

Efficient and Accurate Method for Calculating and Representing Power

Density in the Near Zone of Microwave Antennas

IEEE Trans. Antennas Propagat., Spec. Issue on Near-Field Scanning

Techn., Vol. 36, No. 6, pp. 890-891 June 1988

NEWELL, A. C.; STUBENRAUCH, C. F.

Effect of Random Errors in Planar Near-Field Measurement

IEEE Trans. Antennas Propagat., Spec. Issue on Near-Field scanning

Techn., Vol. 36, No. 6, pp. 769-773

June 1988 
REPJAR, A. G.; NEWELI, A. C.; FRANCIS, M. H. Accurate Determination of Planar Near-Field Correction Parameters for Linearly Polarized Probes IEEE Trans. Ant. Propagat., Special Issue on Near-Field Meas., Vol. 36 , No. 6 , pp. $855-868$ June 1988

MUTH, L. A.

Displacement Errors in Antenna Near-Field Measurements and Their Effect on the Far Field

IEEE Trans. Antennas Propagat., Vol. 36, No. 5, pp. 581-591 May 1988

FRANCIS, M. H.; STUBENRAUCH, C. F.

Comparison of Measured and Calculated Antenna Side Lobe Coupling Loss in the Near Field Using Approximate Far-Field Data

IEEE Trans. Antennas Propagat., Vol. 36, No. 3, pp. 438-441 March 1988

BIII, D. A.

Antennas for Geophysical Applications

Antenna Handbook: Theory, Applications and Design (Chapter 3),

U. T. Lo and S. W. Lee, eds., Van Nostrand Reinhold Co.,

pp. 23-1 through 23-6

1988

LEWIS, R. I.

Spherical-Wave Source-Scattering Matrix Analysis of Coupled Antennas; A General system Two-Port Solution IEEE Trans. Antennas Propagat., Vol. AP-35, No. 12, pp. $1375-1380$

December 1987

LEWIS, R. I.; WITTMANN, R. C.

Improved Spherical and Hemispherical Scanning Algorithms IEEE Trans. Antennas Propagat., Vol. AP-35, No. 12, pp. 1381-1388

December 1987

HILL, D. A.; FRANCIS, M. H.

Out-of-Band Response of Antenna Arrays

IEEE Trans. Electromagn. Compat., Vol. EMC-29, No. 4, pp. 282-288

November 1987

FRANCIS, M. H.; HILL, D. A.

Out-of-Band Response of Array Antennas

Proc., Ant. Meas. Tech. Assoc., 28 september-2 October 1987, Seattle, WA, pp. 14-19

September 1987

MUTH, I. A.

Experimental Study of Interpanel Interactions at $3.3 \mathrm{GHz}$

Proc., Ant. Meas. Tech. Assoc., 28 September-2 October 1987,

Seattle, WA, pp. 25-29

September 1987

REPJAR, A. G.; NEWELI, A. C.; TAMURA, D. T.

Extrapolation Range Measurements for Determining Antenna Gain and Polarization

NBS Tech. Note 1311

August 1987 
HILL, D. A.; FRANCIS, M. H.

Out-of-Band Response of Antenna Arrays

Proc., 1987 IEEE Intl. Symp. Electromagn. Compat.,

25-27 August 1987, Atlanta, GA, pp. 435-438

August 1987

HILL, D. A.; CAVCEY, R. H.

Coupling Between Two Antennas separated by a Planar Interface

IEEE Trans. Geosci. Remote Sensing, Vol. GE-25, No. 4, pp. 422-431 July 1987

DAYWITT, W. C.

A Simple Technique for Determining Joint Losses on a Coaxial Line from Swept-Frequency Reflection Data

IEEE Trans. Instrum. Meas., Vol. IM-36, pp. 468-473

June 1987

FITZGERRELL, R. G.

Three PC-Computer Programs for Antenna Calculations Primarily for Use

Below $1000 \mathrm{MHz}$

IEEE Ant. Propagat. Soc. Newslett., Vol. 29, No. 3, pp. 30-32 June 1987

RANDA, M.; ORR, R. D.

Near-Field Gain of a Horn and an Open-Ended Waveguide; Comparison

Between Theory and Experiment

IEEE Trans. Antennas Propagat., Vol. AP-35, No. 1, pp. 33-40 January 1987

HILL, D. A.

Far-Field Transient Response of an Antenna from Near-Field Data NBSIR 86-3063

December 1986

HILL, D. A.; KOEPRE, G. H.

A Near-Field Array of Yagi-Uda Antennas for Electromagnetic Susceptibility Testing IEEE Trans. Electromagn. Compat., Vol. EMC-28, No. 4, pp. 273-276

November 1986

BAIRD, R. C.; DAYWITT, W. C.; NEWELL, A. C.; PERERA, S.; REPJAR, A. G.; WAIT, D. F.; ESTIN, A. J.

Calibration Requirements for EHF Satellite Communication systems

NBSIR 86-3058

October 1986

RANDA, M.; ORR, R. D.

Near-Field Gain of a Horn and an Open-Ended Waveguide; Comparison Between Theory and Experiment

Proc., 5th Intl. Conf. on Electromagn. Compat., 29 september2 October 1986, University of York, England, pp. 137-145 October 1986

MUTH, L. A.

Gradient Displacement Errors in Antenna Near-Field Measurements and

Their Effects on the Far Field

NBS Tech. Note 1306

october 1986 
FITZGERRELI, R. G.

Standard Iinear Antennas, $30 \mathrm{GHz}$ to $1000 \mathrm{MHz}$ Proc., 5th Intl. Conf. on Electromagn. Compat., 29 September-2 October 1986, University of York, England, pp. 147-153 October 1986

BIIL, D. A.; FRANCIS, M. H.

Out-of-Band Response of Antenna Arrays

NBSIR 86-3047

June 1986

RANDA, M.; ORR, R. D.

Near-Field Gain of a Horn and an Open-Ended Waveguide; Comparison Between Theory and Experiment

Proc., 1986 Intl. IEEE Ant. Propagat Soc. Symp., 8-13 June 1986, Philadelphia, PA, Pp. 91-94 June 1986

NEWELL, A. C.; STUBENRAUCH, C. F.

The Effect of Random Errors in Planar Near-Field Measurements Proc., 1986 Intl. IEEE Ant. Propagat. Soc. Symp., 8-13 June 1986, Philadelphia, PA, pp. 195-198 June 1986

FITZGERREIL, R. G.

Iinear Gain - standard Antennas Below $1000 \mathrm{MHz}$ NBS Tech. Note 1098 May 1986

JESCH, R. I.

Measured Vehicular Antenna Performance

National Institute of Justice, NIJ Report-201-85 May 1986

NEWELI, A. C.; STUBENRAUCB, C. F.; BAIRD, R. C. Calibration of Microwave Antenna Gain Standards

Proc. IEEE, Spec. Issue on Radio Meas. Methods and Standards, Vol. 74, Vol. 1, pp. 129-132 January 1986

LARSEN, E. B.

Calibration and Meaning of Antenna Factor and Gain for EMI Antennas

Interference Technology Engineers' Master (ITEM 1986),

pp. 113-335

1986

MUTH, L. A.

Interelement Interactions in Phased Arrays: Theory, Methods of Data

Analysis, and Theoretical Simulations

NBS Tech. Note 1091

December 1985

YAGEJIAN, A. D.; WITTMANN, R. C.

The Receiving Antenna as a Iinear Differential Operator: Application to Spherical Near-Field Scanning

IEEE Trans. Antenna Propagat., Vol. AP-33, No. 11, pp. 1175-1185

November 1985

NEWELL, A. C.; REPJAR, A. G.

Development of Near-Field Test procedures for Communication Satellite Antennas, Phase I, Part 1

NBSIR 85-3031

September 1985 
HILL, D. A.; KOEPKE, G. H.

An Array of Dipoles for Plane Wave Synthesis

Proc., 1985 Intl. Symp. on Antennas and Electromagn. Theory, 26-28

August 1985, Beijing, China, pp. 422-427

August 1985

MUTH, I. A.

A Theory of Mutual Impedances and Multiple Reflections in an N-Element Array Environment

Proc., 1985 Intl. Symp. Antennas Propagat., 20-22 August 1985,

Kyoto, Japan, pp. 719-722

August 1985

A Theory of Mutual Impedances and Multiple Reflections in an N-Element Array Environment

Proc., 1985 Intl. Symp. Antennas and Electromagn. Theory, 26-28

August 1985, Beijing, China, pp. 440-443

August 1985

HILL, D. A.; ROEPRE, G. H.

A Near-Field Array of Yagi-Uda Antennas for Electromagnetic

Susceptibility Testing

NBS Tech. Note 1082

July 1985

NEWELL, A. C.; FRANCIS, M. H.; RREMER, D. P.; GRIMM, K. R.

Results of Planar Near-field Testing with Ultralow Sidelobe Antennas

Dig., Intl. IEEE Ant. Propagat. Soc. Symp. on Antennas and

Propagat., 17-21 June 1985, University of British Columbia,

Vancouver, Canada, pp. 693-698

June 1985

JESCH, R. L .

Measured Vehicular Antenna Performance

IEEE Trans. Veh. Technol., Vol. VT-34, No. 2, pp. 97-107 May 1985

HILL, D. A.

Out-of-Band Response of Reflector Antennas

NBSIR 85-3021

April 1985

WYSS, J. C.; SHEERAN, S.

A Passive, Optical Modulator and Link for Antennas

IEEE J. Lightwave Technol., Vol. LT-3, No. 2, pp. 316-321 April 1985

CRUZ, J. E.; IARSEN, E. B.

Screenroom Measurements of Antenna Factors

Proc., 1985 Instrum. and Meas. Technol. Conf., 20-22 March 1985,

Tampa, FL, p. 208

March 1985

LARSEN, E. B.; CRUZ, J. E.

$\mathrm{E}$ and $\mathrm{H}$ Fields in Transmission Lines and coils for susceptibility

Testing, Probe Calibration, and RF Exposure Chambers

Proc., 1985 Instrum. and Meas. Technol. Conf., 20-22 March 1985,

Tampa, FL, p. 199

March 1985 
MUTH, I. A.

A Theory of Mutual Impedances and Multiple Reflections in an N-Element Array Environment

$$
\begin{array}{r}
\text { NBS Tech. Note } 1078 \\
\text { February } 1985
\end{array}
$$

KANDA, M.

Transients in a Resistively Loaded Loop Antenna

Proc., 1984 Intl. Symp. on Electromagn. Compat., 16-18 October

1984, Tokyo, Japan, Vol. 1, Pp. 286-290 october 1984

NEWELL, A. C.; FRANCIS, M. H.; RREMER, D. P.

The Determination of Near-Field Correction Parameters for Circularly

Polarized Probes

Proc., 1984 Ant. Meas. Tech. Assoc. Conf.,

2-4 October 1984, Atlanta, GA, Pp. 3A3-1 through 3A3-29

October 1984

STUBENRAUCh, C. F.; FRANCIS, M. H.

Comparison of Measured and Calculated Mutual Coupling in the Near Field Between Microwave Antennas

Proc., 1984 Antenna Applications Symp., 19-21 September 1984,

University of Illinois, Urbana-Champaign, IL, pp. 1-2l September 1984

WYSS, J. C.; ANSON, W. J.; ORR, R. D.

Building Penetration Project

NBSIR 84-3009

September 1984

DAYWITT, W. C.

A Preliminary Investigation into Using the Sun as a Source for $G / T$ Measurements

NBSIR 84-3015

August 1984

KANDA, $\mathbf{M}$.

An Electromagnetic Near-Field Sensor for Simultaneous Electric and Magnetic-Field Measurements

IEEE Trans. Electromagn. Compat., Vol. EMC-26, No. 3,

pp. 102-110

August 1984

STUBENRAUCH, C. F.; FRANCIS, M. H.

Comparison of Measured and Calculated Mutual Coupling in the Near Field Between Microwave Antennas

NBSIR 84-3010

June 1984

WITTMANN, R. C.

Probe Correction in Spherical Near-field Scanning Viewed as an Ideal

Probe Measuring an Effective Field

Dig., 1984 Intl. Symp. Ant. Propagat., 25-29 June 1984, Boston, MA, pp. $674-677$

June 1984

FITZGERREIL, R. G.

A Source of $\mathrm{E}$ and $\mathrm{H}$ Fields for Antenna-Factor Calibration

IEEE Trans. Electromagn. Compat., Vol. EMC-26, No. 2, Pp. 58-65 May 1984 
DAYWITT, W. C.

An Error Analysis for the Use of Presently Available Lunar Radio Flux Data in Broadbeam Antenna-system Measurements NBS Tech. Note 1973

February 1984

HILL, D. A.

Theory of Near-Field Phased Arrays for Electromagnetic susceptibility Testing

NBS Tech. Note 1072

February 1984

MA, M. T.

Arrays of Discrete Elements

Antenna Engineering Handbook, Chapter 3, R. C. Johnson and H. Jasik, eds., McGraw-Hill Book Co. January 1984

GREENLEE, D. H.; RANDA, M.; CHANG, D. C.

The Characteristics of Iris-Fed Millimeter Wave Rectangular Microstrip Patch Antennas

NBS Tech. Note 1063

October 1983

BENSEMA, W. D.

Handbook for Broadband Isotropic Antenna System Volume 1 - Operations Manual

NBSIR 83-1693

July 1983

YAGHJIAN, A. D.

Approximate Formulas for the Far Field and Gain of Open-Ended

Rectangular Waveguide

NBSIR 83-1689

May 1983

REPJAR, A. G.; NEWEIL, A. C.; BAIRD, R. C. Antenna Gain Measurements by an Extended Version of the NBS Extrapolation Method

IEEE Trans. Instrum. Meas., Vol. IM-32, No. 1, pp. 88-91 March 1983

WAIT, D. F.

Precision Measurement of Antenna System Noise Using Radio Stars

IEEE Trans. Instrum. Meas., Vol. IM-32, No. 1, pp. 110-116 March 1983

BAIRD, R. C.; ESTIN, A. J.

The Orbiting Standards Package: A Recalibratable Satellite Instrument Assembly for Measuring Large Earth Station Antennas

Proc., 1982 Ant. Meas. Tech. Assoc., 5-7 October 1982, New Mexico State University, Las Cruces, NM, pp. 5-1 through 5-9 October 1982

FITZGERREIL, R. G.

A Partial Loop Source of $\mathrm{E}$ and $\mathrm{H}$ Fields for Antenna Factor Calibration (A Loop Cell)

Proc., 1982 Ant. Meas. Tech. Assoc., 5-7 October 1982, New Mexico State University, Las Cruces, NM, pp. 15-1 through 15-22

October 1982 
FRANCIS, M. H.; YAGHJIAN, A. D.

Computations of Antenna Side-Lobe Coupling in the Near Field Using

Approximate Far-Field Data

NBSIR 82-1674

August 1982

REPJAR, A. G.; NEWELI, A. C.; BAIRD, R. C.

Antenna Gain Measurements by an Extended Version of the NBS

Extrapolation Method

Dig., 1982 Conf. Prec. Electromagn. Meas., 28 June-1 July 1982, Boulder, CO, pp. F-7 through F-9

June 1982

STUBENRAUCH, C. F.; SPIESS, W.; GAILIANO, P. G.; BABJI, T.

International Intercomparison of Electric Field Strength at $100 \mathrm{MHz}$

Dig., 1982 Conf. Prec. Electromagn. Meas.,

28 June-1 July 1982, Boulder, CO, pp. P-3 through P-4 June 1982

WAIT, D. F.

Precision Measurement of Antenna System Noise Using Radio Stars

Dig., 1982 Conf. Prec. Electromagn. Meas., 28 June-1 July 1982, Boulder, $\mathrm{CO}, \mathrm{p} . \mathrm{F}-17$

June 1982

WYSS, J. C.; KANDA, M.; MELQUIST, D. G.; ONDREJKA, A. R.

Optical Modulator and Link for Broadband Antennas

Dig., 1982 Conf. Prec. Electromagn. Meas.,

28 June-1 July 1982, Boulder, CO, pp. P-16 through P-17

June 1982

RANDA, M.

The Effects of Resistive Loading on TEM Horns

IEEE Trans. Electromagn. Compat., Vol. EMC-24, No. 2,

pp. 245-255

May 1982

RANDA, M.; CHANG, D. C.; GREENLEE, D. H.

The Characteristics of Iris-Fed Millimeter Wave Rectangular Microstrip Patch Antennas

Proc., 1982 IEEE Ant. Propagat. Soc. Symp., 24-28 May 1982,

University of New Mexico, Albuquerque, NM, pp. 292-295 May 1982

REPJAR, A. G.; KREMER, D. P.

Accurate Evaluation of a Millimeter Wave Compact Range Using Planar

Near-Field Scanning

IEEE Trans. Antennas Propagat., Vol. AP-30, No. 3,

pp. 419-425

May 1982

YAGHJIAN, A. D.

A Delta-Distribution Derivation of the Electric Field in the Source Region

J. Electromagn. Soc., Vol. 2, No. 2, pp. 161-167

April-June 1982

WILSON, P. F.; CHANG, D. C.; MA, M. T.

Input Impedance of a Probe Antenna Exciting a TEM Cell

NBS Tech. Note 1054

April 1982 
ESTIN, A. J.; STUBENRAUCH, C. F.; REPJAR, A. G.; NEWEIL, A. C.

Optimized Wavelength-Sized Scalar Horns as Antenna Radiation Standards IEEE Trans. Instrum. Meas., Vol. IM-31, No. 1, pp. 53-56 March 1982

YAGHJIAN, A. D.

Efficient Computation of Antenna Coupling and Fields within the Near-Field Region

IEEE Trans. Antennas Propagat., Vol. AP-30, No. 1, pp. $113-128$

January 1982

TREADO, M. J.; TAGGART, B. E.; NELSON, R. E.; WORKMAN, J. I.

Fixed and Base Station Antennas

National Institute of Justice (NIJ) Standard-0204.01 (Supersedes

National Institute for Law Enforcement and Criminal Justice

(NILECJ) STD-0204.00, dated November 1977)

December 1981

BAIRD, R. C.

Microwave Antenna Measurement Services at the National Bureau of Standards

Dig., Antenna Meas. Tech. Assoc. Mtg.

October 1981

BENSEMA, W. D.

Broadband Orthogonal Array Antenna System: Microprocessor Control and Computation

Record, "EMC '81," 1981 IEEE Int1. Symp. on Electromagn. Compat., 18-20 August 1981, Boulder, CO

August 1981

REEVE, G. R.

Synthesized Isotropic Pattern Antennas for EM Field Measurements

Record, "EMC'81," 1981 IEEE Int1. Symp. on Electromagn. Compat.,

18-20 August 1981, Boulder, CO

August 1981

KERNS, D. M.

Plane-Wave Scattering Matrix Theory of Antennas and Antenna-Antenna

Interactions

NBS MN 162

June 1981

REEVE, G. R.; WAINWRIGHT, A. E.

A Frequency Tracking, Tuned, Receiving Monopole

Dig. 1981 IEEE Ant. Propagat. Soc. Intl. Symp.

June 1981

STUBENRAUCH, C. F.; YAGHJIAN, A. D.

Determination of Mutual Coupling Between Co-sited Microwave Antennas and

Calculation of Near-Zone Electric Field

NBSIR 81-1630

June 1981

LEWIS, R. L.

Spherical Scanning Data Processing: An Algorithm for Halving the Data Processing Effort when the Radiation into the Back Hemisphere is Negligible

Dig., 1981 IEEE Ant. Propagat. Soc. Int 1. Symp., pp. 247-249

June 1981 
LEWIS, R. L. (cont.)

Spherical-Wave Source-Scattering-Matrix Analysis of the Mutual Coupling Between Two Antennas

Dig., 1981 IEEE Ant. Propagat. Soc. Intl. Symp., pp. 261-264 June 1981

Efficient Computation of the Far-Field Radiated by an Arbitrary

Rectangular-Aperture Distribution

NBSIR 81-1643

April 1981

YAGHJIAN, A. D.

A Delta-Distribution Derivation of the Electric Field in the Source Region

Dig., 1981 IEEE Ant. Propagat. Soc. Intl. Symp., pp. 334-337 June 1981

An Approximate Expression for the Principal Beamwidth of Directive Antennas in Terms of Aperture Fields

NBSIR 81-1644

March 1981

MA, M. T.

Synthesis of Broadband Antenna Arrays as Possible Over-the-Horizon

Radars Research Topics in EM Wave Theory

Chapter 9, John Wiley Interscience, pp. 188-209 1981

HIII, D. A.; WAIT, J.R.

Electromagnetic Characteristics of a Coaxial Cable with Periodic slots IEEE Trans. Electromagn. Compat., Vol. EMC-22, No. 4, pp. 303-307 November 1980

WAIT, J. R.; HILI, D. A.

Fields of a Horizontal Loop of Arbitrary Shape Buried in a Two-Layer Earth

Radio Science, Vol. 15, No. 5, pp. 903-912 September-October 1980

RANDA, M.

The Characteristics of a Linear Antenna with Tapered Resistive and Capacitive Loading

Proc., 1980 IEEE Ant. Propagat. Soc. Intl. Symp., 2-6 June 1980, Quebec, Canada, Vol. 2, AP. 18-2, pp. 696-699 June 1980

RANDA, $\mathbf{M}$.

Transients in Resistively Loaded Antennas and Their Comparison with Conical Antennas and TEM Horns

IEEE Trans. Antennas Propagat., Vol. AP-28, No. 1, Pp. 132-136 January 1980

Analytical and Numerical Techniques for Analyzing an Electrically short Dipole with a Nonlinear Load

IEEE Trans. Antennas Propagat., Vol. AP-28, No. 1, pp. 71-78 January 1980

REPJAR, A. G.; KREMER, D. P.

Results of Planar Near-Field Measurements on a Compact Range at 18 and $54 \mathrm{GHz}$

Dig., IEEE Ant. Propagat. Soc. Symp. 1980 
STUBENRAUCH, C. F.

Some Recent Near-Field Antenna Measurements

Proc., 1979 Antenna Application Symp., September, 1979 September 1979

RANDA, M.

The Effects of Resistive Loading on TEM Horns

NBSIR 79-1601

August 1979

MA, M. T.
A Theoretical Study of Unbalanced Ground Effects on Receiving Dipoles NBSIR 79-1605
May 1979

RANDA, M.

The Time Domain Characteristics of a Traveling Wave Linear Antenna with

Linear and Non-Linear Loads

NBSIR 78-892(R)

February 1979

WACKER, P. F.

Plane-Radial Scanning Techniques With Probe Correction; Natural

Orthogonalities with Respect to summation on Planar Measurement Lattices

Dig., IEEE Ant. Propagat. Soc. Symp., pp. 561-564

February 1979

Qualitative Survey of Near-Field Analysis and Measurement

NBSIR 79-1602

January 1979

RANDA, M.

Analytical and Numerical Techniques for Analyzing an Electrically short

Dipole with a Nonlinear Load

NBSIR 78-898

November 1978

IAWTON, R. A.; ONDREJRA, A. R.

Antennas and the Associated Time Domain Range for the Measurement of

Impulsive Fields

NBS Tech. Note 1008

November 1978

TAGGART, H. E.; SHAFER, J. F.

Testing of Electronic Industries Association Land-Mobile Communication

Antenna Gain Standards at the National Bureau of Standards

IEEE Trans. Veh. Technol. Vol. VT-27, pp. 259-264

November 1978

DAYWITT, W. C.

An Error Analysis for the Measurement of Satellite EIRP using a

Calibrated Radio star

IEEE Trans. Instrum. Meas., Vol. IM-27, No. 3, pp. 253-258

September 1978

DOUGHERTY, H. T.; ESTIN, A. J.; MORGAN, W. L.; WOODRUFF, J. J.

The orbiting standards Platform

Proc., 1978 Antenna Applications Symp., 20-22 September 1978,

Urbana-Champaign, IL, pp. 1-9

September 1978 
ESTIN, A. J.; BAIRD, R. C.

Feasibility study of Orbiting standards Platform

NBSIR 78-886

June 1978

DAYWITT, W. C.

Atmospheric Propagation Equations Used in the NBS Earth Terminal

Measurement system

NBSIR 78-883

April 1978

RANDA, M.

A Relatively Short Cylindrical Broadband Antenna with Tapered Resistive Loading for Picosecond Pulse Measurements

Dig., IEEE Antennas Propagat. Soc., pp. 230-233

May 1978

IEEE Trans. Antennas Propagat., Vol. AP-26, No. 3, pp. 439-447 May 1978

WAIT, D. F.

Earth Terminal Measurement System Operations Manual

NBSIR 78-879

April 1978

RANDA, M.

Transients in Resistively Loaded Antennas and Their Comparison with Conical Antennas and TEM Horns NBSIR 78-876

March 1978

DAY, G. W.; STUBENRAUCH, C. F .

Laser Far-Field Beam-Profile Measurements by the Focal Plane Technique NBS Tech. Note 1001 March 1978

DAYWITT, W. C.

Error Equations Used in the NBS Earth-Terminal Measurement system NBSIR 78-869

December 1977

YAGHJIAN, A. D.

A Direct Approach to the Derivation of Electric Dyadic Green's Functions NBS Tech. Note 1000

DAYWITT, W. C.
December 1977

A Precision Earth-Terminal System for Accurate C/KT, G/T, and EIRP

Measurements with a Calibrated Radio star

Proc., 1977 Intl. Union of Radio Sci. (Union Radio Scientifique Internationale) Symp. on Meas. in Telecommunications, 3-7 October 1977, Lannion, France, pp. 1-4

October 1977

MA, M. T.; FITZGERRELI, R. G.

Design of a Van-Top Low-Profile HF Antenna

OTR-77-131

October 1977

YAGHJIAN, A. D.

Near-Field Antenna Measurements on a Cylindrical Surface: A Source Scattering-Matrix Formulation

NBS Tech. Note 696

September 1977 
RANDA, $\mathbf{M}$.

A Relatively short Cylindrical Broadband Antenna with Tapered Resistive Loading for Picosecond Pulse Measurements NBSIR 77-861

August 1977

LEWIS, R. I.

The Use of Three Term Recursion Relations for Numerical Computations as Applied to Near-Field Spherical Scanning Proc., Intl. Union of Radio Science (Union Radio Scientifique Internationale) Intl. Symp., 20-24 June 1977, pp. 224-226 June 1977

WACKER, P. F.

Recent Results with Spherical Near-Field Antenna scanning at the National Bureau of Standards Proc., 1977 European Space Agency Symp., 6-8 June 1977, Noordwijk, Netherlands, pp. 159-164 June 1977

WACKER, P. F.; NEWELL, A. C. Advantages and Disadvantages of Planar, Circular Cylindrical and Spherical Scanning and Description of the NBS Antenna Scanning Facilities

Proc., 1977 European Space Agency Symp., 6-8 June 1977, Noordwijk, Netherlands, pp. 115-121 June 1977

WAIT, D. F.

Satellite Earth Terminal G/T Measurements

Microwave J., Vol. 20, No. 4, pp. 49, 51, 58 April 1977

WACKER, P. F.

Symmetry Analysis Applied to scattering, Inverse scattering, and Antenna Patterns: Measurements, Moment Method, and Characteristic Modes

Proc., 1977 Intl. IEEE Ant. Propagat. Soc. Symp., pp. 177-180 1977

Symmetry Analysis Applied to Wave Theory

Proc., Intl. IEEE Ant. Propagat. Soc. Symp., pp. 169-172 1977

YAGHJIAN, A. D.

A Direct Approach to the Derivation of Electric Dyadic Green's Functions Dig., Ant. Propagat. Soc. Symp., pp. 76-156

October 1976

DAYWITT, W. C.

Error Equations Used in the NBS Precision G/T Measurement System NBSIR 76-842

September 1976

RANDA, $\mathbf{M}$.

Study of Error Analysis for Absolute Flux Density Measurements of Cassiopeia

IEEE Trans. Instrum. Meas., Vol. IM-25, No. 3, pp. 173-182 September 1976 
LARSEN, E. B .; ANDREWS, J . R.

Using Fiber Optics in a Broadband, Sensitive, Isotropic Antenna $15 \mathrm{kHz}$ to $150 \mathrm{MHz}$

Proc., IEEE Intl. Symp. on Electromagn. Compat., 13-15 July 1976, Washington, DC, pp. 385-389 July 1976

CRAWFORD, M. I.

Calibration of Broadbeam Antennas Using Planar Near-Field Measurements Proc., 1976 Conf. on Prec. Electromagn. Meas., 28 June-1 July 1976, Boulder, CO, Pp. 53-56 June 1976

NEWELL, A. C.; REPJAR, A. G.

Results of Spherical Near-Field Measurements on Narrow-Beam Antennas Proc., 1976 Conf. on Prec. Electromagn. Meas., 28 June-1 July 1976, Boulder, CO, Pp. 382-385 June 1976

YAGHJIAN, A. D.

Upper-Bound Errors in Far-Field Antenna Parameters Determined from

Planar Near-Field Measurements. Part 1: Analysis

NBS Tech. Note 667

October 1975

DAYWITT, W. C.; RANDA, M.

G/T Measurement Errors with Radio Stars

Proc., 1975 IEEE Intl. Antennas and Propagat. Symp., 2-4 June 1975, Urbana-Champaign, IL, Session 20, pp. 460-463 June 1975

NEWELL, A. C.

Improved Polarization Measurements Using a Modified Three Antenna Technique

Proc., 1975 IEEE Intl. Antennas and Propagat. Symp., 2-4 June 1975, Urbana-Champaign, IL, Session 15, pp. 337-340 June 1975

NEWEIL, A. C.; YAGHJIAN, A. D.

Study of Errors in Planar Near-Field Measurements Proc., 1975 IEEE Intl. Antennas and Propagat. Symp., 2-4 June 1975, Urbana-Champaign, IL, Session 20, pp. 470-473 June 1975

KANDA, $\mathbf{M}$.

Accuracy Considerations in the Measurement of the Power Gain of a Large Microwave Antenna

IEEE Trans. Antennas Propagat., Vol. AP-23, No. 3, pp. 407-411

May 1975

RERNS, D. M.

Plane-Wave Scattering Matrix Theory of Antennas and Antenna-Antenna

Interactions: Formulation and Applications

NBSIR 75-824

January 1975 
WACRER, P. F.

Non-Planar Near-Field Measurements: Spherical Scanning

NBSIR 75-809

1975

NEWELL, A. C.; CRAWFORD, M. L.

Planar Near-Field Measurements on High Performance Array Antennas NBSIR 74-380

July 1974

BUSSEY, H. E.; LARSEN, E. B.

Buried Antenna Performance: Development of Small Resonant Buried Antennas

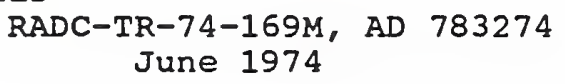

RANDA, M.

Accuracy Considerations in the Measurement of the Power Gain of a Iarge Microwave Antenna

Proc., 1974 Intl. Ant. Propagat. Soc. Symp., 10-12 June 1974,

Atlanta, GA, $\mathrm{pp} .43-45$

June 1974

NEWEIL, A. C.; CRAWFORD, M. I.

Planar Near-Field Measurements on Phased Array Antennas

Proc., 1974 Intl. IEEE Ant. Propagat. Soc. Symp., 10-12 June 1974, Atlanta, GA, p. 423 June 1974

WACRER, P. F.

Antenna Measurements at the National Bureau of Standards Near-Field and Extrapolation Techniques

Proc., 1974 Colloquium on Microwave Communication 24-30 June 1974, Budapest, PME-113-114

June 1974

BAIRD, R. C.; KERNS, D. M.

The Accurate Determination of Millimeter-Wave Antenna Characteristics by Deconvolution and Extrapolation Techniques

Proc., 1974 Millimeter-Wave Conf., 26-28 March, 1974, Vol. 2,

pp. E 2-1 through E 2-12

March 1974

WACKER, P. F.

Near-Field Antenna Measurements Using a Spherical Scan: Efficient Data

Reduction with Probe correction

Dig., Conf. Prec. Electromagn. Meas., No. 113, pp. 286-288 London, England

1974

NEWELL, A. C.; BAIRD, R. C.; WACKER, P. F.

Accurate Measurement of Antenna Gain and Polarization at Reduced

Distances by an Extrapolation Technique

IEEE Trans. Antennas Propagat., Vol. AP-21, No. 4, pp. 418-431 July 1973

KERNS, D. M.

Comment on Correction of Errors in Aerial Far-Field Radiation-Pattern

Determinations

Electronics Lett., Vol. 7, No. 24, pp. 706

December 1971 
NEWELL, A. C.; RERNS, D. M.

Determination of Both Polarization and Power Gain of Antennas by a Generalized 3-Antenna Measurement Method

Electronics Lett., Vol. 7, No. 3, pp. 68-70

January 1971 
BAKER-JARVIS, J.R.; JANEZIC, M. D.; STAFFORD, R. B.

Shielded Open-Circuited Sample Holders for Dielectric and Magnetic

Measurements of Liquids and Powders

NISTIR 5001

March 1993

CLARK, A. V.; SCHAPS, S. R.; BARER-JARVIS, J.R.; GEYER, R. G. Development of Electromagnetic Probes for Intelligent Processing of Dielectric Materials NISTIR 4963, pp. 34-37

November 1992

BARER-JARVIS, J. R.; GEYER, R. G.; DOMICH, P. D.

A Nonlinear Least-Squares Solution with Causality Constraints Applied to Transmission Line Permittivity and Permeability Determination

IEEE Trans. Instrum. Meas., Vol. 41, No. 5, pp. 646-652 October 1992

BARER-JARVIS, J. R.

Dielectric and Magnetic Measurement Methods in Transmission Lines: An Overview

Proc., AMTA Workshop on EM Characterization of Materials for Antenna/RCS Applications, 20-25 July 1992, Chicago, IL, pp. 1-23 July 1992

GEYER, R. G.; BAKER-JARVIS, J.R.; JANEZIC, M. D.; DOMICH, P. D. Spectral Characterization of Ferrites for Use as Magnetic Reference Materials

Digest, 1992 Conf. Prec. Electromagn. Meas., 9-12 June 1992,

Paris, France, pp. 107-108

June 1992

VANZURA, E. J.; WEIL, C. M.; WIILIAMS, D. F.

Complex Permittivity Measurements of Gallium Arsenide Using a HighPrecision Resonant Cavity

Digest, 1992 Conf. Prec. Electromagn. Meas., 9-12 June 1992,

Paris, France, pp. 103-104 June 1992

BARER-JARVIS, J. R.; JANEZIC, M. D.; GROSVENOR, J. H.; GEYER, R. G. Transmission/Reflection and Short-Circuit Line Methods for Measuring Permittivity and Permeability NIST TN 1355 May 1992

WEIL, C. M.

The Electromagnetic Properties of Materials Program at NIST

Proc., Mat. Soc. Symp., 27-30 April 1992, San Francisco, CA, Vol. 269, pp. 517-526

April 1992

CLARK, A. V.; SCHAPS, S. R ; BARER-JARVIS, J.R.; GEYER, R . G •;

CAPOBIANCO, T. E.; AULD, B. A.

Development of Electromagnetic Probes for Intelligent Processing of

Dielectric Materials

NISTIR 4693, pp. 54-61

November 1991

DOMICH, P. D.; BAKER-JARVIS, J . R .; GEYER, R. G.

Optimization Techniques for Permittivity and Permeability Determination NIST JRES, Vol. 96, No. 5, pp. 565-575

September/October 1991 
DOMICH, P. D.; BARER-JARVIS, J.R.; GEYER, R. G. (cont.)

Optimization Techniques for Permittiivity and Permeability Determination NISTIR 4571

June 1991

JANEZIC, M. D.; GROSVENOR, J. H.

Improved Technique for Measuring Permittivity of Thin Dielectrics with a Cylindrical Resonant Cavity

Conf. Record, IEEE Instrum. Meas. Tech. Conf., 14-16 May 1991, Atlanta, GA, pp. 580-584 May 1991

WEIL, C. M.; RISSICK, W. A.

The NIST Electromagnetic Properties of Materials Program

Conf. Record, IEEE Instrum. Meas. Tech. Conf., 14-16 May 1991, Atlanta, GA, pp. 626-630 May 1991

VANZURA, E. J.; ROGERS, J. E.

Evaluation of a Resonant Circuit Model Using Reflected S-Parameter Data Conf. Record, IEEE Instrumentation and Measurement Technology Conf., 14-16 May 1991, Atlanta, GA, pp. 150-155 May 1991

BARER-JARVIS, J.R.; VANZURA, E. J.; RISSICK, W. A. Improved Technique for Determining Complex Permittivity with the Transmission/Reflection Method

IEEE Trans. Microwave Theory Tech., Vol. 38, No. 8, pp. 1096-1103 August 1990

BARER-JARVIS, J . R.

Transmission/Reflection and short-Circuit Line Permittivity Measurement Methods

NIST Tech. Note 1341

July 1990

BAKER-JARVIS, J. R •; GEYER, R. G .

Improvements in Transmission Line Permittivity and Permeability Measurements

Dig., Conf. Prec. Electromagn. Meas., 11-14 June 1990, Ottawa, Canada, pp. 232-233

June 1990

GEYER, R. G.; WEIL, C. M.; KISSICK, W. A.

Precision Dielectric Measurements Using a Mode-Filtered Cylindrical Cavity Resonator

Dig., Conf. Prec. Electromagn. Meas., 11-14 June 1990, Ottawa, Canada, pp. 174-175 June 1990

GEYER, R. G.

Dielectric Characterization and Reference Materials

NIST Tech. Note 1338

April 1990

Electrodynamics of Materials for Dielectric Measurement Standardization Record, Instrum. Meas. Tech. (IMTC) Conf., 13-15 February 1990, San Jose, CA, pp. 2-7

February 1990 
HILL, D. A.

Electric and Magnetic Dipole Radiation in a Random Medium

Electromagnetics, Vol. 10, pp. 279-292 1990

GEYER, R. G.; JANEZIC, M. D.; KISSICR, W. A.; BARER-JARVIS, J. R.

Preliminary Microwave Complex Permittivity Measurements of Heavy-Metal Fluoride Glasses

Proc., Third DOD Symp. on Electromagnetic Windows, 14-16 November

1989, Redstone Arsenal, Huntsville, AL, pp. 116-141 November 1989

HIIL, D. A.

Near-Field Detection of Buried Dielectric objects

IEEE Trans. Geosci. Remote Sensing, Vol. GE-27, No. 4, pp. 364-368 July 1989

VANZURA, E. J.; KISSICK, W. A.

Advances in NIST Dielectric Measurement Capability Using a Mode-Filtered Cylindrical cavity

Proc., 1989 IEEE MTT-S Intl. Microwave Symp., 13-15 June 1989,

Long Beach, CA, pp. 901-904

June 1989

JESCH, R. I.

Measurement of Shielding Effectiveness of Different Cable and Shielding Configurations by Mode-stirred Techniques

IEEE Trans. Electromagn. Compat., Vol. EMC-30, pp. 222-228 August 1988

GEYER, R. G .

Dielectric Mixing Rules for Background Test Soils

NBSIR 88-3095

June 1988

LAWTON, R. A.; ANDERSON, W. T.

Two Layer Dielectric Microstrip Line Structure: $\mathrm{SiO}_{2}$ on $\mathrm{Si}$ and GaAs on

Si: Modeling and Measurement

IEEE Trans. Microwave Theory Tech., Vol. MTT-36, No. 4, pp. 785-789

April 1988

DAYWITT, W. C.

Complex Permittivity of Beryllium Oxide Between 100 and 300 Kelvins at 9.3 Gigahertz

IEEE Trans. Instrum. Meas., Vol. IM-34, No. 1, pp. 98-99 March 1985

JESCH, R. I.

Dielectric Measurements of oil Shale as Functions of Temperature and Frequency

IEEE. Trans. Geosci. Remote Sensing, Vol. GE-22, No. 2, pp. 99-105 March 1984

BUSSEY, H. E.

Dielectric Measurements in a shielded open circuit coaxial Line

IEEE Trans. Instrum. Meas., Vol. IM-29, No. 2, pp. 120-124 June 1980 
BUSSEY, H. E.

Dielectric Measurements of Lunar Soil

Proc., 1979 Lunar and Planetary Science Conf. (10th) Natl.

Aeronautics and Space Administration, 19-23 March 1979,

Lyndon B. Johnson Space Center, Houston, TX

March 1979

Open Circuited Coaxial Resonator for High Sensitivity Dielectric Measurements, Application to Lunar soil 70051-20

Proc., 1979 Lunar and Planetary Science Conf. (10th) Natl. Aeronautics and Space Administration, 19-23 March 1979,

Lyndon B. Johnson Space Center, Houston, TX March 1979

BENNETT, W. S .; TAGGART, H. E.

Characteristics of A CISPR/VDE Far-Field EMI Test site with Ground screen

Proc., Third Rotterdam Electromagn. Compat. Symp., December 1978

JONES, R. N.; BUSSEY, H. E.; LITTLE, W. E.; METZRER, R. F.

Electrical characteristics of Corn, wheat, and soya in the 1-200 MHz Range

NBSIR 78-897

October 1978

JESCH, R. I.

Dielectric Measurements of Five Different Soil Textural Types as

Functions of Frequency and Moisture Content

NBSIR 78-896

1978

ELLERBRUCH, D. A.; LITILE, W. E.; BOYNE, H. S.; BACHMAN, D. D.

Microwave Characteristics of snow

Proc., 1977 Western Snow Conf., 18-21 April 1977,

Albuquerque, $N M, p 7$

April 1977

BUSSEY, H. E.; RICHMOND, J. H.

scattering by a Lossy Dielectric Circular Cylindrical Multilayer, Numerical Values

IEEE Trans. Antennas Propagat., Vol. 23, No. 5, pp. 723-725 September 1975

BUSSEY, H. E.; MORRIS, D.; ZALTSMAN, E. B.

International Comparison of Complex Permittivity Measurement at $9 \mathrm{GHz}$ IEEE Trans. Instrum. Meas., Vol. IM-23, No. 3, pp. 235-239 September 1974

LAWTON, R. A.

Autocorrelation and Power Measurement with Pyroelectric and Dielectric Bolometers

IEEE Trans. Instrum. Meas., IM-22, Pp. 299-306

December 1973

BUSSEY, H. E.

Wavelength of a slotted Rectangular Iine containing Two Dielectrics NBSIR 73-326

July 1973 
BUSSEY, H. E. (cont.)

Rapport sur la Comparaison Int'l des Mesures de Permittivite Complexe a

$9 \mathrm{GHz}$ (Intl. Comparison of Complex Permittivity Measurement at $9 \mathrm{GHz}$ ) Proc., of Comite Consultatif, D'Electricite Comite, Bureau Intl. des Poids et Mesures, 12-13 October 1972, Sevres, France, pp. 124-137

October 1972 
HIIL, D. A.; CRAWFORD, M. L.; KANDA, M.

Apeture Coupling to Shielded Transmission Lines: Theory and Experiment Proc. 10th Intl. Zurich Symp. on EMC, 9-11 March 1993, Zurich, Switzerland, pp. 569-571 March 1993

BIII, D. A.; CRAWFORD, M. I.; KANDA, M.; WU, D. I. Aperture Coupling to a Coaxial Air Line: Theory and Experiment IEEE Trans. Electromagn. Compat., Vol. 35, No. 1, pp. 69-74 February 1993

RANDA, J. P.

Correction Factor for Nonplanar Incident Field in Monopole Calibrations IEEE Trans. Electromagn. Compat., Vol. 35, No. 1, pp. 94-96 February 1993

BIL工, D. A.

Currents Induced on Multiconductor Transmission Lines by Radiation and Injection

IEEE Trans. Electromagn. Compat., Vol. 34, No. 4, pp. 445-450 November 1992

ADAMS, J. W.

Electromagnetic Shielding of RF Gaskets Measured by Two Methods

Record, 1992 IEEE Int1. Symp. on EMC, 17-21 August 1992, Anaheim, CA, pp. 154-157

August 1992

ADAMS, J. W.; CRUZ, J. E.; MELQUIST, D. G.

Comparison Measurements of Currents Induced by Radiation and Injection IEEE Trans. Electromagn. Compat., Vol. 34, No. 3, pp. 360-362 August 1992

CRAWFORD, M. L.; RIDDLE, B. F.

A Reverberating asymmetric TEM Cell for Radiated EMC/V and SE Testing, $10 \mathrm{kHz}-18 \mathrm{GHz}$

Record, 1992 IEEE Intl. Symp. on EMC, 17-21 August 1992, Anaheim, CA, pp. 206-213

August 1992

KOEPKE, G. H.; DRIVER, L. D.; CAVCEY, R. H.; MASTERSON, K. D.; JOBNR, R. T.; RANDA, $M$.

New Spherical Dipole Source

Record, 1992 IEEE Intl. Symp. On EMC, 17-21 August 1992, Anaheim, CA, pp. 98-105

August 1992

RANDA, J. P.; RANDA, M.; ORR, R. D.

Optimized Thermo-Optic Electric-Field Probes for Microwaves and

Millimeter Waves

Record, 1992 IEEE Intl. Symp. on EMC, 17-21 August 1992, Anaheim, CA, pp. 200-203 August 1992

TOFANI, S.; ONDREJRA, A. R.; RANDA, M.; HILL, D. A. Bistatic Scattering of Absorbing Materials from 30 to $1000 \mathrm{MHz}$

IEEE Trans. Electromagn. Compat., Vol. 34, No. 3, pp. 304-307 August 1992

HIIL, D. A.

Gradiometer Antennas for Tunnel Detection NISTIR 3990

June 1992 
MA, M. T.

EMC Standards and Regulations: A Brief Review NISTIR 3989

May 1992

MA, M. T.; ADAMS, J. W.

System Response to Pulsed Excitations Estimated from Measurement of $\mathrm{CW}$ Amplitudes

Proc., Intl. Symp. on Electromagn. Compat., 25-27 May 1992,

Beijing, China, pp. 29-32 May 1992

HILL, D. A.; CRAWFORD, M. L.; RANDA, M.; WU, D. I.

Aperture Coupling to Shielded Transmission Lines: Theory and Experiment NISTIR 3988

April 1992

RANDA, M.; EILI, D. A.

A Three-Loop Method for Determining the Radiation Characteristics of an Electrically Small Source

IEEE Trans. Electromagn. Compat., Vol. EMC-34, No. 1, pp. 1-3 February 1992

RANDA, M.; MASTERSON, R. D.

Optically Sensed EM-Field Probes for Pulsed Fields

Proc., IEEE Spec. Issue on Antennas, Vol. 80, No. 1, pp. 209-215 January 1992

BILI, D. A.

Electromagnetic Scattering by a Periodic Surface with a Wedge Profile Electromagnetics, vol. 12, pp. 247-264 1992

KOEPKE, G. H.; DRIVER, L. D.; CAVCEY, R. H.; MASTERSON, R. D.; JOHNK, R. T.; RANDA, M.

A Standard Spherical Dipole Source

NIST Tech. Note 1351

December 1991

MA, M. T.; ADAMS, J. W.

Phase Characteristics and Time Responses of Unknown Linear Systems

Determined from Measured CW Amplitude Data

NIST Tech. Note 1349

November 1991

MA, M. T.; LARSEN, E. B.; CRAWFORD, M. L.

Electromagnetic Fields with Arbitrary Wave Impedances Generated Inside a TEM Cell

IEEE Trans. Electromagn. Compat., Vol. EMC-33, No. 4, pp. 358-362 November 1991

RANDA, J. P.; RANDA, M.; ORR, R. D.

Thermo-Optic Designs for Electromagnetic Field Probes for Microwaves and Millimeter Waves

IEEE Trans. Electromagn. Compat., Vol. EMC-33, No. 3, pp. 205-314 August 1991

Resistively-Tapered-Dipole Electric-Field Probes up to $40 \mathrm{GHz}$

Proc., IEEE Intl. Electromagn. Compat. Symp. 13-15 August 1991

Cherry Hill, NJ, pp. 265-266

August 1991 
HILL, D. A.

Diffraction by a Half Plane in a Lossy Medium

J. Appl. Phys., Vol. 69, No. 12, pp. 8405-8407 June 1991

ONDREJKA, A. R.; KANDA, M.

A Time-Domain Method for Measuring the Reflection Coefficient of Microwave Absorbers at Frequencies Below $1 \mathrm{GHz}$

Dig., 1991 Ant. Prop. Soc. Symp., 24-28 June 1991, London, ontario, Canada, vol. 3, pp. 1656-1663 June 1991

CRAWFORD, M. L.; RIDDLE, B. F.

A Proposed TEM Driven Mode-Stirred Chamber for Large System Radiated EMC/V Testing $10 \mathrm{kHz}-40 \mathrm{GHz}$

Proc., 9th Intl. Symp. on Electromagn. Compat. 12-14 March 1991, Zurich, Switzerland, pp. 431-437 March 1991

RANDA, M.; RANDA, J. P.

Estimation of Electromagnetic Fields in Complex Environments

- Proc., 9th Intl. Symp. on Electromagn. Compat., 12-14 March 1991, Zurich, Switzerland, pp. 337-342 March 1991

RANDA, J. P. Simultaneous vs. Independent Injection Testing of Nonlinear Multiport systems

Proc., 9th Intl. Symp. on Electromagn. Compat., 12-14 March 1991, Zurich, Switzerland, pp. 71-74 March 1991

HILI, D. A.

A Generalization of the Cornu spiral for Lossy Media

J. Appl. Phys., Vol. 69, No. 3, pp. 1772-1774 February 1991

WILSON, P. F.; MA, M. T.

Fields Radiated by Electrostatic Discharges

IEEE Trans. Electromagn. Compat., Vol. EMC-33, No. 1, pp. 10-18 February 1991

CAMELI, D. G.; LARSEN, E. B.; CRUZ, J. E.; HILL, D. A. NIST Calibration Procedure for Vertically Polarized Monopole Antennas, $30 \mathrm{kHz}$ to $300 \mathrm{MHz}$

NIST Tech. Note 1347

January 1991

HILL, D. A.

Near-Field and Far-Field Excitation of a Long Conductor in a Lossy Medium

NISTIR 3954

September 1990 
HILL, D. A. (cont.)

Quasi-Static Analysis of a Two-Wire Transmission Line Located at an

Interface

Radio Science, Vol. 25, No. 4, pp. 435-440

July-August 1990

CRAWFORD, M. L.; MA, M. T.; LADBURY, J. M.; RIDDLE, B. F.

Measurement and Evaluation of a TEM/Reverberating Chamber

NIST Tech. Note 1342

July 1990

CRAWFORD, M. L.; LADBURY, J. M.; RIDDLE, B. F.; LARSEN, E. B.

Electromagnetic Radiation Test Facilities: Evaluation of a Small

Reverberating Chamber Located at RADC, Griffiss AFB, Rome, NY NISTIR 90-3939

June 1990

MASTERSON, R. D.; KANDA, M.

Broadband, Photonic Electric Field Sensors for EMP and HPM Applications Proc., Fifth Natl. Conf. on High Power Microwave Technology (DoD), 10-15 June 1990, West Point, NY, pp. 388-391 June 1990

ADAMS, J. W.; WU, D. I.; BUDLONG, A.

Measurement of Electric Field Strength Near Higher-Powered Personal

Transceivers

NISTIR 90-3938

May 1990

HILL, D. A.; KANDA, M.; LARSEN, E. B.; KOEPRE, G. H.; ORR, R. D.

Generating Standard Reference Electromagnetic Fields in the NIST

Anechoic Chamber, 0.2 to $40 \mathrm{GHz}$

NIST Tech. Note 1335

March 1990

RANDA, M.

A Microstrip Patch Antenna as a Standard Transmitting and Receiving Antenna.

IEEE Trans. Electromagn. Compat., Vol. 32, No. 1, pp. 5-8 February 1990

RANDA, J. P.

Theoretical Considerations for a Thermo-Optic Microwave Electric-Field Probe

J. Microwave Power Electromagn. Energy, Vol. 25, No. 3, pp. 133140 1990

MA, M. T.; CRAWFORD, M. L.

Facilities for Improving Evaluations of Electromagnetic Susceptibilities of Weapon Systems and Electronic Equipment

NISTIR 89-3928

November 1989

WU, D. I.; RANDA, M.

Comparison of Theoretical and Experimental Data for the Near-Field of an Open Ended Rectangular Waveguide

IEEE Trans. Electromagn. Compat., Vol. EMC-31, No. 4, pp. 353-358 November 1989 
RANDA, M.

A Microstrip Patch Antenna as a Standard Transmitting and Receiving Antenna

Dig., 1989 Intl. Symp. on Electromagn. Compat., 8-10 September

1989, Nagoya, Japan, pp. 460-462

September 1989

MASTERSON, R. D.; DRIVER, L. D.

A Broadband, Isotropic, Photonic Electric Field Probe for Measurements from $10 \mathrm{kHz}$ to Above $1 \mathrm{GHz}$

Proc., High Bandwith Analog Applications of Photonics II, SPIE Intl. Soc. of Optical Engineers, Vol. 987

8-9 September 1988, Boston, MA, pp. 107-118 September 1989

CRAWFORD, M. I.

TEM/Reverberating Chamber Design/Concept Study: A Single Facility for

Large system Radiated EMC Testing, $10 \mathrm{kHz}-40 \mathrm{GHz}$ ?

Proc., EMC EXPO, 1-3 August 1989, Washington, DC,

pp. B6.22-B6.29

August 1989

FITZGERREIL, R. G.

Monopole Impedance and Gain Measurements of Finite Ground Planes

National Institute of Justice Report, 200-87 May 1989

IARSEN, E. B.; EERET, R. L.; CAMELI, D. G.; ROEPRE, G. H.

Calibration of Antenna Factor at a Ground Screen Field Site Using an Automatic Network Analyzer

Proc., IEEE $1989 \mathrm{Natl}$. Symp. on Electromagn. Compat., 23-25 May

1989, Denver, CO, pp. 19-24 May 1989

MASTERSON, K. D.; DRIVER, L. D.; RANDA, M.

Photonic Probes for the Measurement of Electromagnetic Fields over Broad Bandwidths

Proc., IEEE $1989 \mathrm{Natl}$. Symp. on Electromagn. Compat., 23-25 May

1989, Denver, CO, pp. 1-6 May 1989

RANDA, J. P.; RANDA, M.; MELQUIST, D. G.; ORR, R. D.

Thermo-optic Designs for Microwave and Millimeter-wave Electric-Field Probes

Proc., IEEE $1989 \mathrm{Nat}$. Symp. on Electromagn. Compat., 23-25 May

1989, Denver, CO, pp. 7-11

May 1989

WU, D. I.; CBANG, D. C.

The Effect of an Electrically Large Stirrer in a Mode-stirred Chamber IEEE Trans. Electromagn. Compat., Vol. EMC-31, No. 2,

pp. 164-169

May 1989

ROEPRE, G. H.; MA, M. T.; BENSEMA, W. D. Implementation of an Automated System for Measuring Radiated Emissions Using a TEM cell

IEEE Trans. Instrum. Meas., Vol. IM-38, No. 2, pp. 473-479

April 1989 
CRUZ, J. E.; LARSEN, E. B.

Alternative Techniques for some Typical MIL-sTD-461/462 Types of Measurements

NBS Tech. Note 1320

March 1989

MA, M. T.

How High is the Level of Electromagnetic Fields Radiated by an ESD? Proc., 8th Intl. Symp. on Electromagn. Compat., 7-9 March 1989, Zurich, Switzerland, pp. 361-365 March 1989

CAVCEY, $\mathbf{R}$. H.

Transmission Loss Through 6061 T-6 Aluminum Using a Pulsed Eddy Current source

"Materials Evaluation", American Society for Nondestructive

Testing, Vol. 47 , No. 2, pp. 216-218 February 1989

HILI, D. A.

Clutter Models for Subsurface Electromagnetic Applications

NISTIR 89-3909 February 1989

Reflection Coefficient of a Waveguide with Slightly Uneven Walls

IEEE Trans. Microwave Theory Tech., Vol MTT-37, No. 1, pp. 244-252 January 1989

ROEPKE, G. H.; MA, M. T.; BENSEMA, W. D.

Theory and Measurements of Radiated Emissions Using a TEM Cell

NIST Tech. Note 1326 January 1989

ADAMS, J. W.; FRIDAY, D. S.

Measurement Procedures for Electromagnetic Compatibility Assessment of Electroexplosive Devices

IEEE Trans. Electromagn. Compat., Vol. EMC-30, No. 4, pp. $484-494$

November 1988

DRIVER, L. D.; RANDA, M.

An Optically Linked Electric and Magnetic Field sensor for Poynting Vector Measurements in the Near Fields of Radiating Sources

IEEE Trans. Electromagn. Compat., Vol. EMC-30, No. 4, Pp. 495-503 November 1988

MASTERSON, R. D.; DRIVER, L. D. A Broadband, Isotropic, Photonic Electric Field Probe for Measurements from $10 \mathrm{kHz}$ to Above $1 \mathrm{GHz}$ Proc., High Bandwith Analog Applications of Photonics II, SPIE Intl. Soc. of Optical Engineers, Vol. 987, 8-9 September 1988, Boston, MA, pp. 107-118 September 1989

REEVE, G. R.

Proficiency Testing for MIL-STD 462 NVLAP Laboratories

Dig., EMC EXPO'88, 10-12 May 1988, washington, DC, Pp. T33.13

through T33.15

september 1988 
WU, D. I. ; CHANG, D. C.

A Hybrid Representation of the Green's Function in an Over-Moded Rectangular Cavity

IEEE Trans. Microwave Theory Tech., Vol. MTT-36, No. 9, pp. 1334-1342

September 1988

CAMELL, D. G.; LARSEN, E. B.; ANSON, W. J.

NBS Calibration Procedures for Horizontal Dipole Antennas

( 25 to $100 \mathrm{MHz}$ )

Proc. IEEE Intl. Symp. on Electromagn. Compat., 2-4 August 1988 ,

Seattle, WA, pp. 390-394

August 1988

CRAWFORD, M. L.; LADBURY, J. M.

Mode-Stirred Chamber for Measuring Shielding Effectivess of Cables and Connectors an Assessment of MIL-STD 1344A Method 3008

Proc., 1988 IEEE Intl. Symp. on Electromagn. Compat., 1-5 August

1988, Seattle, WA, pp. 30-36

August 1988

JESCB, R. I.

Measurement of Shielding Effectiveness of Different Cable and Shielding Configurations by Mode-stirred Techniques

IEEE Trans. Electromagn. Compat., Vol. EMC-30, pp. 222-228 August 1988

RANDA, M.; ORR, R. D.

Generation of Standard Electromagnetic Fields in a TEM Cell

NBS Tech. Note 1319

August 1988

WILSON, P. F.; MA, M. T.; ADAMS, J. W.

Techniques for Measuring the Electromagnetic Shielding Effectivenss of

Materials: Part I -- Far-Field Source Simulation

IEEE Trans. Electromagn. Compat., Vol. EMC-30, No. 3, pp. 239-250 August 1988

WILSON, P. F.; MA, M. T.

Techniques for Measuring the Electromagnetic Shielding Effectiveness of Materials: Part II -- Near-Field Source Simulation

IEEE Trans. Electromagn. Compat., Vol. EMC-30, No. 3, pp. 251-259 August 1988

WILSON, P. F.; MA, M. T.; ONDREJKA, A. R.

Fields Radiated by Electrostatic Discharges Proc., IEEE Intl. Symp. on Electromagn. Compat., 2-4 August 1988, Seattle, WA, pp. 179-183

August 1988

RANDA, M. ; DRIVER, L. D.

An Optically Linked Electric and Magnetic Field Sensor for Poynting Vector Measurements in the Near Field of Radiating Sources

Dig., Conf. Prec. Electromagn. Meas., 7-10 June 1988, Tsukuba

Science City, Japan, pp. 32-33

June 1988 
MA, M. T.

Theory and Measurements of Unintentional Radiators

Dig., Conf. Prec. Electromagn. Meas., 7-10 June 1988, Tsukuba Science City, Japan, pp. 30-31 June 1988

RANDA, J. P.; RANDA, M.; MELQUIST, D. G.; SEGA, R. M.; NORGARD, J. D. High Frequency Electric Field Probe Development Proc., EMC EXPO'88, Int1. Conf. on Electromagn. Compat., 10-12 May 1988, Washington, DC, pp. T15.31 through T15.37 May 1988

VANZURA, E. J. Automated System for Electromagnetic Field Generation and Immunity Testing

Proc., 1988 IEEE Instrum. Meas. Technol. Conf., 19-22 April 1988, San Diego, CA, pp. 3-10 April 1988

MA, M. T.

Understanding Reverberating Chambers as an Alternative Facility for EMC Testing

J. Electromagn. Waves App1., Vo1. 2, pp. 339-351 March/April 1988

FITZGERRELL, R. G. Monopole Impedance and Gain Measurements on Finite Ground Planes

IEEE Trans. Antennas Propagat., Vol. 36, No. 3, pp. 431-438 March 1988

WU, D. I.; CHANG, D. C.

The Effect of a Large Rotating Scatterer in a Rectangular Cavity NBS Tech. Note 1317 March 1988

BILI, D. A.

A Circular Array for Plane-Wave Synthesis

IEEE Trans. Electromagn. Compat., Vol. EMC-30, No. 1, pp. 3-8 February 1988

RANDA, J. P.; RANDA, M.; MELQUIST, D. G.

Possible Designs for Electric-Field Strength-Probes for Millimeter Waves NBSIR 88-3084

February 1988

WILSON, P. F.

A Comparison Between Near-Field Shielding Effectiveness Measurements

Based on Coaxial Dipoles and on Electrically Small Apertures

IEEE Trans. Electromagn. Compat., Vol. EMC-30, No. 1, pp. 23-28 February 1988

WILSON, P. F.; ONDREJRA, A. R.; MA, M. T.; LADBUUY, J. M. Electromagnetic Fields Radiated From Electrostatic Discharges - Theory and Experiment NBS Tech. Note 1314

February 1988

CAVCEY, K. H.; FRIDAY, D. S.

Aircraft Field Degradation and Electromagnetic Compatibility NBSIR 88-3083

January 1988 
HIIL, D. A.

Electromagnetic Detection of Long Conductors in Tunnels

Proc., Third Tunnel Detection Symp., 12-15 January 1988,

Golden, CO, pp. 518-537

January 1988

CRAWFORD, M. L.; KOEPKE, G. H.; LADBURY, J. M.

EMR Test Facilities Evaluation of Reverberating Chamber Located at RADC, Griffiss, AFB, Rome, New York

NBSIR $87-3080$

December 1987

RANDA, J. P.; RANDA, M.

A New Approach to Volumes Irradiated by Unknown Sources

IEEE Trans. Electromagn. Compat., Vol. EMC-29, No. 4, pp. 273-281 November 1987

VANZURA, E. J.; ADAMS, J.W.

Generating Constant Electromagnetic Fields Inside a Partially-Ioaded

Shielded Room

Test and Meas. World, pp. 72-83

November 1987

JESCE, R. I.

Measurement of Shielding Effectiveness of Different Cable and Shielding Configurations by Mode-stirred Techniques

NBSIR 87-3076

October 1987

MA, M. T.

Characterization of Unknown RF Leakage Sources: Problems, Solutions and Practical Implications

Union Radio Scientifique Internationale XXII General Assembly, 24

August - 2 September 1987, Tel Aviv, Israel

September 1987

WU, D. I.; CHANG, D. C.

An Investigation of a Ray-Mode Representation of the Green's Function in a Rectangular Cavity

NBS Tech. Note 1312

September 1987

CRAWFORD, M. I.

A TEM Driven Reverberating Chamber: A Single Facility for Radiated

EMS/V Testing, $10 \mathrm{kHz}-18 \mathrm{GHz}$ ?

Proc., EMC Expo 1987, Intl. Conf. on Electromagn. Compat.,

19-21 May 1987, San Diego, CA, pp. T11.18 through T11.28

May 1987

MA, M. T.; BENSEMA, W. D.

Automated TEM Cell for Measuring Unintentional EM Emissions

Proc., EMC Expo 1987, Int1. Conf. on Electromagn. Compat.,

19-21 May 1987, San Diego, CA, pp. Tl1.1 through T11.12

May 1987

CRAWFORD, M. L.; ROEPRE, G. H.

Performing EM Susceptibility/Vulnerability Measurements Using a Reverberation Chamber

Proc., 7th Intl. Symp. on Electromagn. Compat., 3-5 March 1987,

Zurich, Switzerland, pp. 121-126

March 1987 
MARTZLOFF, F. D.; WILSON, P. F.

Fast Transient Tests - Trivial or Terminal Pursuit?

Proc., 7th Intl. Symp. on Electromagn. Compat., 3-5 March 1987, Zurich, Switzerland, pp. 283-288 March 1987

RANDA, J. P.; RANDA, M.

A Lattice Approach to Environments Irradiated by Unknown Sources

Proc., 7th Intl. Symp. Electromagn. Compat., 3-5 March 1987, Zurich, Switzerland, pp. 191-195 March 1987

WILSON, P. F.; MA, M. T.

Techniques for Measuring the Shielding Effectiveness of Materials Proc., 7th Intl. Symp. on Electromagn. Compat., 3-5 March 1987, Zurich, Switzerland, pp. 547-552 March 1987

RANDA, M.; DRIVER, L. D.

An Isotropic, Electric-Field Probe with Tapered Resistive Dipoles for Broadband Use, $100 \mathrm{kHz}-18 \mathrm{GHz}$

IEEE Trans. Microwave Theory Tech., Vol. MTT-35,

No. 2 , pp. 124-130

February 1987

HII工, D. A.

Effect of a Thin Conducting sheet on the Fields of a Buried Magnetic Dipole

Electromagnetics, pp. 71-79 1987

CRAWFORD, M. I.; BEAN, J. I.

NSWC Reverberating Chamber: A High Power Microwave Exposure Chamber Proc., 3rd Natl. High Power Microwave Technical Conf., 1-5 December 1986, Kirtland AFB, NM, p. 5 December 1986

HII工, D. A.

An Error Bound for Near-Field Array Synthesis

IEEE Trans. Electromagn. Compat., Vol. EMC-28, No. 4, pp. 273-276

November 1986

HII工, D. A.; ROEPKE, G. H.

A Near-Field Array of Yagi-Uda Antennas for Electromagnetic Susceptibility Testing

IEEE Trans. Electromagn. Compat., Vol. EMC-28, No. 4, pp. 273-276

November 1986

BENSEMA, W. D.; ROEPRE, G. H .; MEDLEY, H. W.

Handbook for NBS Multisensor Automated EM Field Measurement system NBSIR 86-3056

October 1986

CRUZ, J. E.; LARSEN, E. B.

Assessment of Errors for MIL-STD-461/462

NBS Tech. Note 1300

October 1986 
JESCE, R. L.

A Survey of Triaxial and Mode-stirred Techniques for Measuring the Shielding Effectiveness of Connectors and Cables NBSIR 86-3060 October 1986

RANDA, M.; WYSS, J. C.

Evaluation of Off-Axis Measurements Performed in an Anechoic Chamber NBS Tech. Note 1305 October 1986

RANDA, J. P.; RANDA, M.

A Lattice Approach to Volumes Irradiated by Unknown Sources NBS Tech. Note 1303 October 1986

ADAMS, J. W.; VANZURA, E. J. Shielding Effectiveness Measurements of Plastics EMC Technology and Interference Control News, pp. 39-44 September-October 1986

CRAWFORD, M. L.; ROEPRE, G. B.

Preliminary Evaluation of Reverberation Chamber Method for Pulsed RF Immunity Testing Record, IEEE Intl. Symp. on Electromagn. Compat., 16-18 september 1986, San Diego, CA, pp. 270-278 September 1986

RANDA, M.; DRIVER, L. D. An Isotropic, Electric-Field Probe with Tapered Resistive Dipoles for Broadband Use, $100 \mathrm{kHz}-18 \mathrm{GHz}$ Record, IEEE Intl. Symp. on Electromagn. Compat., 16-18 September 1986, San Diego, CA, pp. 256-261 September 1986

RANDA, M.; ORR, R. D.

Near-Field Gain of a Horn and an Open-Ended Waveguide: Comparison Between Theory and Experiment Proc., 5th Intl. Conf. on Electromagn. Compat., 29 September-2 October 1986, University of York, England, pp. 137-145

September 1986

MA, M. T.; RANDA, M.; CRAWFORD, M. L.; IARSEN, E. B. Measuring Electromagnetic Interference, Part I, Open-Field Sites and TEM Cells; Part II, Reverberating Chambers; Part III, Anechoic Chambers and Field Probes

Test and Measurement World, Part I, pp. 72-88, February 1986; Part II, pp. 74-90, May 1986, Part III, pp. 81-99, september 1986 September 1986

RANDA, J. P.; RANDA, M.

A Lattice Approach to Complex Electromagnetic Environments Record, IEEE Intl. Symp. on Electromagn. Compat., 16-18 September 1986, San Diego, CA, pp. 329-331

September 1986 
WILSON, P. F.; MA, M. T. Shielding Effectiveness Measurements Using an Apertured TEM Cell in a Reverberation Chamber

Record, IEEE Intl. Symp. on Electromagn. Compat., 16-18 September 1986, San Diego, CA, pp. 265-269 September 1986

MA, M. T.

Shielding Effectiveness Measuring Using an Apertured TEM Cell in a Reverberation Chamber

Record, IEEE Intl. Symp. on Electromagn. Compat., 16-18 September 1986, San Diego, CA September 1986

BILL, D. A.

Out-of-Band Response of a Coax-to-Waveguide Adapter

IEEE Trans. Electromagn. Compat., Vol. EMC-28, No. 3, pp. 156-158

August 1986

WILSON, P. F.; MA, M. T.

Simple Approximate Expressions for Higher-Order Mode Cut off and Resonant Frequencies in TEM Cells

IEEE Trans. Electromagn. Compat., Vol. EMC-28, No. 3, pp. $125-130$

August 1986

HILL, D. A.; WAIT, J.R.

Anomalous Vertical Magnetic Field for Electromagnetic Induction in a Laterally Varying Thin Conductive sheet

Radio science, Vol. 21, No. 4, pp. 617-621

July-August 1986

MA, M. T.; RANDA, M.

Electromagnetic Compatibility and Interference Metrology

NBS Tech. Note 1099

July 1986

CRAFFORD, M. L.; ROEPKE, G. H.

Performing EM Susceptibility/Vulnerability Measurements Using a

Reverberation Chamber

Proc., EMC EXPO 1986, Intl. Conf. on Electromagn. Compat.,

16-19 June 1986, Washington, DC, Pp. T28.7 through T28.14

June 1986

EMR Test Facilities - Evaluation of Reverberation Chambers Located at

NSWC, Dahlgren, VA

NBSIR 86-3051

June 1986

RANDA, M.; DRIVER, L. D.

A Broadband, Electric-Field Probe Using Resistively Tapered Dipoles, 100

$\mathrm{kHz}-18 \mathrm{GHz}$

Proc., 1986 IEEE/MTT/S Intl. Microwave Symp. Dig.,

2-4 June 1986, Baltimore, MD, pp. 621-624 June 1986

REEVE, G. R.

Alternate EMI Measurement Techniques for Microelectronic Circuits

Proc., EMC EXPO 1986, Intl. Conf. on Electromagn. Compat., 16-19

June 1986, Washington, DC, pp. T26.1 through T26.4

June 1986 
WILSON, P. F.; MA, M. T.

Methods for Measuring the Near-Field and Far-Field Shielding

Effectiveness of Materials

Proc., EMC Expo 1986 Intl. Conf. on Electromagn. Compat., 16-19

June 1986, Washington, DC, pp. T28.1-T28.6

June 1986

FRIDAY, D. S.; ADAMS, J.W.

A Statistical Characterization of Electroexplosive Devices Relevant to Electromagnetic Compatibility

NBS Tech. Note 1094

May 1986

JESCH, R. I.

Susceptibility of Emergency Vehicle Sirens to External Radiated

Electromagnetic Fields

National Institute of Justice Technology Program, NIJ

Report-200-85

May 1986

WIISON, P. F.; MA, M. T.

A Study of Techniques for Measuring the Electromagnetic Shielding

Effectiveness of Materials

NBS Tech. Note 1095

May 1986

CRAWFORD, M. L.; KOEPKE, G. H.

Design, Evaluation and Use of a Reverberation Chamber for Performing

Electromagnetic Susceptibility/Vulnerability Measurements

NBS Tech. Note 1092

April 1986

KUFFEL, J •; MALEWSKI, R.; VAN HEESWIJK, R .; LAWTON, R. A.

Dynamic Performance of Digital Recorders Used for Monitoring

High-Voltage Impulse Tests

Proc., IEEE Instrum. Meas. Technol. Conf., 25-27 March 1986,

Boulder, Co, Vol. IM-35, No. 4, pp. 591-595 March 1986

FITZGERRELL, R. G.

Site Attenuation

IEEE Trans. Electromagn. Compat., Vol. EMC-28, No. 1, pp. 38-40 February 1986

HIIL, D. A.

Electromagnetic Wave Propagation in an Asymmetrical Coal Seam

IEEE Trans. Antennas Propagat., Vol. AP-34, No. 2,

pp. 244-247

February 1986

WIISON, P. F.; MA, M. T.

Electromagnetic Shielding Effectiveness: Measurement Techniques and Interpretations

1986 IEEE Regional Conf. and Exhib. on Electromagn. Compat.,

6 February 1986, Anaheim, CA, pp. 1-14

February 1986 
ADAMS, J. W.; VANZURA, E. J.

Shielding Effectiveness Measurements of Plastics

NBSIR 85-3035

January 1986

FRIDAY, D. S.

Methodology for Statistical Control of the Anechoic Chamber Field

Generation System

NBSIR 85-3033

January 1986

KANDA, M.; LARSEN, E. B.; BORSERO, M.; GALLIANO, P. G.; YODOSHIMA, I.; NAHMAN, N. S.

Standards for Electromagnetic Fields Measurements

Proc., IEEE Spec. Issue on Radio Meas. Methods and Standards, Vol.

74, No. 1 , pp. 120-128

January 1986

MA, M. T.

Measurements of Unintentional Electromagnetic Emissions

Proc., IEEE Spec. Issue on Radio Meas. Methods and Standards, Vol.

74, No. 1, pp. 110-111 January 1986

WILSON, P. F.; MA, M. T.

Measurements of Electromagnetic Shielding Capabilities of Materials Proc., IEEE Spec. Issue on Radio Meas. Methods and Standards, Vol. 74, No. 1, pp. 112-115 January 1986

HIIL, D. A.

Radio-Wave Propagation from a Forest to a clearing

Electromagnetics, pp. 217-228 1986

LARSEN, E. B.

Calibration and Meaning of Antenna Factor and Gain for EMI Antennas Interference Technology Engineers' Master (ITEM 1986), pp. 113-335

1986

MASTERSON, R. D.

A Photonic Electric Field Probe for Frequencies up to $2 \mathrm{GHz}$

Proc., Soc. for Photo-Optical Instrumentation Engineers (SPIE), Vol. 720 , p. 100 1986

NAHMAN, N. S ; RANDA, M.; LARSEN, E. B.; CRAWFORD, M. I. Methodology for Standard Electromagnetic Field Measurements IEEE Trans. Instrum. Meas., Vol. IM-34, No. 4, pp. 490-503 December 1985

FITZGERRELL, R. G.

Site Attenuation

NBS Tech. Note 1089

November 1985

HILL, D. A.

A Numerical Method for Near-Field Array Synthesis

IEEE Trans. Electromagn. Compat., Vol. EMC-27, No. 4, pp. 201-211

November 1985 
CRUZ, J. E.; DRIVER, L. D.; RANDA, M.

Design of the National Bureau of Standards Isotropic Magnetic Field

Meter (MFM-10) $300 \mathrm{kHz}$ to $100 \mathrm{MHz}$

NBS Tech. Note 1085

October 1985

WILSON, P. F.; CHANG, D. C.

Mode Coupling by a Longitudinal slot for a class of Planar Waveguiding Structures: Part I - Theory

IEEE Trans. Microwave Theory Tech., Vol. MTT-33,

No. 10, pp. 981-987

October 1985

Mode Coupling by a Longitudinal slot for a class of Planar Waveguiding

Structures: Part II - Applications

IEEE Trans. Microwave Theory Tech., Vol. MTT-33,

No. 10 , pp. $988-993$

October 1985

CRAWFORD, M. L.; KOEPKE, G. H.

Comparing EM Susceptibility Measurement Results Between Reverberation

and Anechoic Chambers

Record, IEEE 1985 Intl. Symp. on Electromagn. Compat.,

20-22 August 1985, Wakefield, MA, pp. 200-202

August 1985

FITZGERRELL, R. G.

Site Attenuation

Record, IEEE 1985 Intl. Symp. on Electromagn. Compat.,

20-22 August 1985, Wakefield, MA, pp. 612-617

August 1985

HILL, D. A.; KOEPRE, G. H.

An Array of Dipoles for Plane Wave Synthesis

Proc., 1985 Intl. Symp. on Antennas and Propagat.,

20-22 August 1985, Kyoto, Japan, pp. 177-180

August 1985

RANDA, M.; ORR, R. D.

A Radio-Frequency Power Delivery system: Procedures for Error Analysis and Self-Calibration

NBS Tech. Note 1083

August 1985

RANDA, J. P.; RANDA, M.

A Finite-Element Action Approach to the Characterization of Complex

Electromagnetic Environments

Proc., 1985 Intl. Symp. on Antennas and EM Theory,

26-28 August 1985, Beijing, China, pp. 48-53

August 1985

Directional Scanning of Complex Electromagnetic Environments Proc., 1985 Intl. Symp. on Antennas and Propagat.,

20-22 August 1985, Kyoto, Japan, pp. 899-902

August 1985

High Frequency Errors of an Electric-field-meter in Complicated Environments

Record, IEEE 1985 Intl. Symp. on Electromagn. Compat.,

20-22 August 1985, Wakefield, MA, pp. 618-621

August 1985 
WILSON, P. F.; MA, M. T.

Factors Influencing Material Shielding Effectiveness Measurements Record, IEEE 1985 Intl. Symp. on Electromagn. Compat., 20-22 August 1985, Wakefield, MA, pp. 29-33 August 1985

Shielding Effectiveness Measurements in a Dual TEM Cell IEEE Trans. Electromagn. Compat., Vol. EMC-27, No. 3, pp. 137-142

August 1985

HILL, D. A.; ROEPKE, G. H.

A Near-field Array of Yagi-Uda Antennas for Electromagnetic Susceptibility Testing

NBS Tech. Note 1082 July 1985

RANDA, J. P.; RANDA, M.

A Directional Scanning Technique for characterization of complex Electromagnetic Environments Dig., 1985 IEEE Ant. Propagat. Soc. Symp., 17-21 June 1985, University of British Columbia, Vancouver, BC, Canada, pp. 521-524 June 1985

WYSS, J. C.; SHEERAN, S.

A Passive, Optical Modulator and Link for Antennas

IEEE J. Lightwave Technol., Vol. IT-3, No. 2, pp. 316-321 April 1985

BENSEMA, W. D.; REEVE, G. R.; KOEPKE, G. H.

A Multisensor Automated EM Field Measurement System Proc., 1985 Instrum. and Meas. Technol. Conf., 20-22 March 1985, Tampa, FL, pp. 200-202 March 1985

CRUZ, J. E.; LARSEN, E. B.

Screenroom Measurements of Antenna Factors Proc., 1985 Instrum. and Meas. Technol. Conf., 20-22 March 1985, Tampa, FL, p. 208 March 1985

RANDA, M.

A Methodology for Evaluating Microwave Anechoic Chamber Measurements Proc., 1985 symp. and Technical Exhib. on Electromagn. Compat., 5-7 March 1985, Zurich, Switzerland, pp. 69-74 March 1985

RANDA, M.; NABMAN, N. S.

Standards for Measurement of Electromagnetic Fields Proc., 1985 Instrum. and Meas. Technol. Conf., 20-22 March 1985, Tampa, FL, pp. 20-23 March 1985

RANDA, M.; RANDA, J. P.; NAHMAN, N. S .

Possible Estimation Methodologies for Electromagnetic Field Distributions in Complex Environments NBS Tech. Note 1081 March 1985 
LARSEN, E. B.; CRUZ, J. E.

$E$ and $H$ Fields in Transmission Lines and Coils for Susceptibility

Testing, Probe Calibration, and RF Exposure Chambers

Proc., 1985 Instrum. and Meas. Technol. Conf.,

20-22 March 1985, Tampa, FL, p. 199

March 1985

MA, M. T.; RANDA, M.; CRAWFORD, M. I.; LARSEN, E. B.

A Review of Electromagnetic Compatibility/Interference Measurement Methodologies

Proc., IEEE, Vol. 73, No. 3, pp. 388-411 March 1985

RANDA, J. P.; RANDA, M.

Multiple-Source, Multiple-Frequency Error of an Electric Field Meter IEEE Trans. Antennas Propagat., Vol. AP-33, No. 1, pp. 2-9 January 1985

WILSON, P. F.; MA, M. T.

Input Impedance of a Probe Antenna in a TEM Cell

IEEE Trans. Electromagn. Compat., Vol. EMC-26, No. 4 , pp. 154-161

November 1984

RANDA, $M$.

Error Analysis of Radiation Characteristics of an Unknown Interference Source Based on Power Measurements

Proc., 1984 Intl. Symp. on Electromagn. Compat.,

16-18 October 1984, Tokyo, Japan, pp. 39-44

October 1984

WILSON, P. F.; MA, M. T.

Small Aperture Analysis of the Dual TEM Cell and an Investigation of

Test object scattering in a single TEM Cell

NBS Tech. Note 1076

October 1984

Small obstacle Loading in a TEM Cell

Proc., 1984 Intl. Symp. on Electromagn. Compat.,

16-18 October 1984, Tokyo, Japan, pp. 30-35

october 1984

WYSS, J. C.; ANSON, W. J.; ORR, R. D.

Building Penetration Project

NBSIR 84-3009

September 1984

ONDREJRA, A. R.; ADAMS, J.W.

Shielding Effectiveness (SE) Measurement Techniques

Proc., 1984 EMI/RFI Technical Conf., The Soc. of Plastics

Engineers, Inc., 18-20 June 1984, Chicago, IL, pp. 19-26 June 1984

SHAFER, J. F.

Field Strength Levels in Vehicles Resulting from Communications

Transmitters

National Institute of Justice Report, 200-83

1984 
WILSON, P. F.; MA, M. T.

Some Problems Associated with Interpreting Shielding Effectiveness Measurement Results

Proc., 1984 EMI/RFI Technical Conf., The Soc. of Plastics Eng.,

Inc., 18-20 June 1984, Chicago, IL, pp. 9-18 June 1984

FITZGERRELI, R. G.

A Source of $E$ and $H$ Fields for Antenna-Factor Calibration

IEEE Trans. Electromagn. Compat., Vol. EMC-26, No. 2, pp. 58-65 May 1984

CRAWFORD, M. L.; KOEPKE, G. H.

Operational Considerations of a Reverberation Chamber for EMC Immunity Measurements, Some Experimental Results

Proc., 1984 IEEE Natl. Symp. on Electromagn. Compat.,

24-26 April 1984, San Antonio, TX, pp. 47-54

April 1984

DRIVER, I. D.; REEVE, G. R.

Application of a Systematic Approach to an Investigation of $\mathrm{HF}$

Interference to a Shipboard Radar set

Proc., 1984 IEEE Natl. Symp. on Electromagn. Compat., 24-26 April

1984, San Antonio, TX, pp. 211-218

April 1984

ONDREJRA, A. R.; ADAMS, J. W.

Shielding Effectiveness (SE) Measurement Techniques

Proc., 1984 IEEE Natl. Symp. on Electromagn. Compat.,

24-26 April 1984, San Antonio, TX, pp. 249-256

April 1984

WILSON, P. F.; MA, M. T.

Small Aperture Analysis of the Dual TEM Cell

Proc., 1984 IEEE Natl. Symp. on Electromagn. Compat.,

24-26 April 1984, San Antonio, TX, pp. 365-369

April 1984

HILL, D. A.

Theory of Near-Field Phased Arrays for Electromagnetic susceptibility Testing

NBS Tech. Note 1072

February 1984

ALSPACH, W. J.; MILLER, C. R. S.; REEVE, G. R.

An Assessment of Electromagnetic Interference (EMI) and Electromagnetic Compatibility (EMC) Measurement Practices to Meet U.S. Army Aviation

Research and Development Command EMI/EMC Requirements

NBSIR 84-1698(R)

January 1984

RANDA, M.

Arrays of Discrete Elements

Antenna Engineering Handbook, Chapter 3, R. C. Johnson and H. Jasik, eds., McGraw-Hill Book Co.

January 1984 
CRAWFORD, M. I.

Comparison of Open-Field, Anechoic Chamber and TEM Cell

Facilities/Techniques for Performing Electromagnetic Radiated Emissions Measurements

Record, IEEE 1983 Intl. Symp. on Electromagn. Compat., 23-25

August 1983, Arlington, VA, pp. 413-418 August 1983

FITZGERRELI, R. G.

E-Fields Over Ground

Record, IEEE 1983 Intl. Symp. on Electromagn. Compat., 23-25

August 1983, Arlington, VA, pp. 6-9 August 1983

IIU, B. H.; CHANG, D. C.; MA, M. T.

Eigenmodes and the Composite quality Factor of a Reverberating Chamber NBS Tech. Note 1066

August 1983

Design Consideration of Reverberating Chambers for Electromagnetic Interference Measurements

Record, IEEE 1983 Intl. Symp. on Electromagn. Compat., 23-25

August 1983, Arlington, VA, pp. 508-512 August 1983

WILSON, P. F.; CHANG, D. C.; MA, M. T.; CRAWFORD, M. L.

Theoretical and Experimental Analysis of Coupling Characteristics of Dual TEM Cells

Record, IEEE 1983 Intl. Symp. on Electromagn. Compat., 23-25

August 1983, Arlington, VA, pp. 513-517

August 1983

BENSEMA, W. D.

Handbook for Broadband Isotropic Antenna System

Volume 1 - Operations Manual

NBSIR 83-1693

July 1983

MA, M. T.; ROEPKE, G. H.

Uncertainties in Extracting Radiation Parameters for an Unknown

Interference Source Based on Power and Phase Measurements NBS Tech. Note 1064

June 1983

RANDA, M.; RIES, F. $\mathbf{Z}$.

Time Domain Sensors for Radiated Impulsive Measurements

IEEE Trans. Antennas Propagat., Vol. AP-31, No. 3, pp. 438-444 May 1983

KANDA, M.

An Electric and Magnetic Field Sensor for Simultaneous Electromagnetic Near-Field Measurements - Theory

NBS Tech. Note 1062

April 1983 
CRAWFORD, M. L.

Improving the Repeatability of EM Susceptibility Measurements of Electronic Components When Using TEM Cells

SAE Technical Paper Series, 830607, International Congress and Exposition, 28 February-4 March 1983, Detroit, MI, pp. 1-8 March 1983

Evaluation of Shielded Enclosure for EMI/EMC Measurements Without and With RF Anechoic Material

Proc., 1983 Electromagn. Compat. Symp. and Exhib., 8-10 March

1983, Zurich, Switzerland, pp. 397-402 March 1983

RANDA, M.; RIES, F. X.; DRIVER, L. D.; ORR, R. D.

An Electric and Magnetic Field Sensor Concept for Simultaneous

Near-Field Electromagnetic Components when Using TEM Cells

Proc., 1983 Electromagn. Compat. Symp. and Exhib., 8-10 March

1983, Zurich, Switzerland, pp. 263-266

March 1983

KOEPKE, G. H.; MA, M. T.

A New Method for Determining the Emission Characteristics of an Unknown

Interference Source

Proc., 1983 Electromagn. Compat. Symp. and Exhib., 8-10 March

1983, Zurich, Switzerland, pp. 263-266

March 1983

STUBENRAUCH, C. F.; SPIESS, W.; GALLIANO, P. G.; BABJI, T.

International Intercomparison of Electric Field Strength at $100 \mathrm{MHz}$

IEEE Trans. Instrum. Meas., Vol. IM-32, No. 1, pp. 235-237 March 1983

MILIER, C. K. S.

The EMI Measurement Challenge

Proc., 1983 Meas. Sci. Conf., 20-21 January 1983,

Palo Alto, CA, pp. 189-197

January 1983

FITZGERREII, R. G.

A Partial Loop Source of E\&H Fields for Antenna Factor Calibration (A Loop Cell)

Proc., 1982 Antenna Meas. Techniques Assoc, 5-7 October 1982, New Mexico State University, Las Cruces, NM, pp. 15-1 through 15-22 October 1982

MA, M. T.; KOEPRE, G. H.

A Method to Quantify Radiation Characteristics of an Unknown

Interference source

NBS Tech. Note 1059

October 1982

TAGGART, H. E.

Radiated EMI Instrumentation Errors

EMC Technology Magazine, Vol. 1, No. 4, pp. 26-35 October 1982

DRIVER, L. D.; CRUZ, J. E.

Development of the NBS Isotropic Magnetic-Field Meter (MFM-10), $300 \mathrm{kHz}$ to $100 \mathrm{MHz}$

Proc., 1982 Intl. Symp. on Electromagn. Compat., 8-10 september

1982, Santa Clara, CA, pp. 460-467

September 1982 
RANDA, M.; RIES, F. X.

Time Domain Sensors for Radiated Impulsive Measurements

Proc., IEEE 1982 Intl. Symp. on Electromagn. Compat.,

8-10 September 1982, Santa Clara, CA, pp. 296-301 september 1982

KOEPRE, G. H.; MA, M. T.

A New Method for Determining the Emission Characteristics of an Unknown Interference source

Proc., IEEE 1982 Intl. Symp. on Electromagn. Compat.,

8-10 september 1982, Santa Clara, CA, pp. 151-156

September 1982

ADAMS, J. W.

Measurement of Electromagnetic Radiation from Electric Rail Cars

NBSIR 82-1669

August 1982

FITZGERRELL, R. G.

Free-Space Transmission Loss for Anechoic Chamber Performance Evaluation

IEEE Trans. Electromagn. Compat., Vol. EMC-24, No. 3, pp. 356-358 August 1982

RANDA, M.; RIES, F. X.; DRIVER, L. D.; ORR, R. D.

Design Considerations for Broadband Magnetic-Field Sensors

Dig., 1982 Conf. Prec. Electromagn. Meas., 28 June-1 July 1982,

Boulder, CO, pp. $\mathrm{P}-11$ through $\mathrm{P}-13$

June 1982

WYSS, J. C.; RANDA, M.; MELQUIST, D. G.; ONDREJRA, A. R.

Optical Modulator and Link for Broadband Antennas

Dig., 1982 Conf. Prec. Electromagn. Meas., 28 June-1 July 1982,

Boulder, CO, pp. $\mathrm{P}-16$ through $\mathrm{P}-17$

June 1982

RANDA, M.

The Effects of Resistive Loading on TEM Horns

IEEE Trans. Electromagn. Compat., Vol. EMC-24, No. 2, pp. 245-255 May 1982

ARTHUR, M. G.; ORR, R. D.; REEVE, G. R.

Planning Guidance for Future EMI Measurement Instrumentation

NBSIR 82-1662

April 1982

WILSON, P. F.; CHANG, D. C.; MA, M. T.

Input Impedance of a Probe Antenna Exciting a TEM Cell

NBS Tech. Note 1054

April 1982

MA, M. T.; ARTHUR, M. G.

A Study of Distribution of Electromagnetic Fields Inside Buildings with Apertures Excited by an External source

NBSIR 82-1659

February 1982

RIES, F. X.; MILLER, C. R. S.

Influence of Electromagnetic Interference on Electronic Devices Bulletin OIMI Seminar

December 1981 
TAGGART, H. E.

Methods of Suppressing Automotive Interference NBS SP 480-44

November 1981

BENSEMA, W. D.

Broadband Orthogonal Array Antenna System: Microprocessor Control and Computation

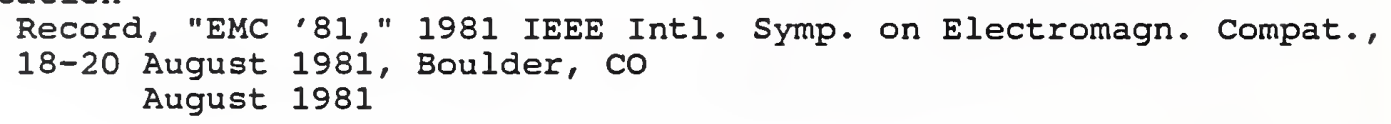

CRUZ, J. E.

Design, Construction, and Calibration of the Broadband Electric Field Monitor (EFM-5)

Record, "EMC '81," 1981 IEEE Intl. Symp. on Electromagn. Compat., 18-20 August 1981, Boulder, Co

August 1981

FITZGERRELI, R. G.

Free-Space Transmission Loss for Anechoic Chamber Performance Evaluation Record, "EMC '81," 1981 IEEE Intl. Symp. on Electromagn. Compat., 18-20 August 1981, Boulder, Co

August 1981

LARSEN, E. B.

Background and Present Status of RF Probe Development at NBS

Record, "EMC '81," 1981 IEEE Intl. Symp. on Electromagn. Compat., 18-20 August 1981, Boulder, Co

August 1981

REEVE, G. R.

Synthesized Isotropic Pattern Antennas for EM Field Measurements

Record, "EMC'81," 1981 IEEE Intl. Symp. on Electromagn. Compat., 18-20 August 1981, Boulder, Co

August 1981

SREENIVASIAH, I.; CHANG, D. C.; MA, M. T.

A Critical Study of Emission and Susceptibility Levels of Electrically

Small objects from Tests Inside a TEM Cell

Record, "EMC '81," 1981 IEEE Intl. Symp. on Electromagn. Compat.,

18-20 August 1981, Boulder, CO

August 1981

Emission Characteristics of Electrically Small Radiating Sources from

Tests Inside a TEM Cell

IEEE Trans. Electromagn. Compat., Vol. EMC-23, No. 3,

pp. 113-121

August 1981

CLARK, H. E.

Requirements for an Effective National Nonionizing Radiation Measurement System

NBS SP 613

June 1981

REEVE, G. R.; WAINWRIGHT, A. E.

A Frequency Tracking, Tuned, Receiving Monopole

Record, IEEE/APS Symp., June 1981

June 1981 
RANDA, M.

Theoretical and Experimental Investigations of Electromagnetic Field Distortion Due to a Perfectly Conducting Rectangular Cylinder in a Transverse Electromagnetic Cell

NBS Tech. Note 1028

April 1981

MA, M. T.; CHANG, D. C.; SREENIVASIAH, I.

A Method of Determining the Emission and Susceptibility Levels of

Electrically Small objects Using a TEM Cell

NBS Tech. Note 1040

April 1981

CRAWFORD, M. L.

Options to Open-Field and Shielded Enclosure Electromagnetic

Compatibility Measurements

Proc., 4th Symp. Electromagn. Compat., 10-12 March 1981, Zurich, Switzerland

March 1981

RANDA, M.

Theoretical and Experimental Investigations of Loading Effects Due to a Perfectly Conducting Rectangular Cylinder in a Transverse

Electromagnetic Cell

Proc., 4th Symp. Electromagn. Compat., 10-12 March 1981, Zurich, Switzerland

March 1981

LARSEN, E. B.; RIES, F. Z.

Design and Calibration of the NBS Isotropic Electric-Field Monitor

$(E F M-5), 0.2$ to $1000 \mathrm{MHz}$

NBS Tech. Note 1033

March 1981

MA, M. T.; WILSON, P. F.; CHANG, D. C.

Excitation of a TEM Cell by a Vertical Electric Hertzian Dipole NBS Tech. Note 1037

March 1981

CRAWFORD, M. I.

Evaluation of a Reverberation Chamber Facility for Performing EM

Radiated Fields Susceptibility Measurements

NBSIR $81-1638$

February 1981

BOWMAN, R. R.

A Temperature Probe for Radio-Frequency Heated Material NBSIR 81-1634

January 1981

HILL, D. A.; WAIT, J. R.

Electromagnetic Characteristics of a Coaxial Cable with Periodic slots IEEE Trans. Electromagn. Compat., Vol. EMC-22,

No. 4, pp. 303-307

November 1980

MILIER, C. R. S.

Challenges of EMI Measurements

Proc., Natl. Conf. of Standards Laboratories,

22-25 September 1980 , pp. 8-1 through 8-17

September 1980 
CRAWFORD, M. L.; WORKMAN, J. I.

Spherical Dipole for Radiating Standard Fields

Dig., 1980 Conf. Prec. Electromagn. Meas., 23-27 June 1980,

Braunschweig, FDR, pp. 424-429

June 1980

RANDA, M.

The Characteristics of a Linear Antenna with Tapered Resistive and Capacitive Loading

Proc., IEEE Ant. Propagat. Soc. Symp., 2-6 June 1980, Quebec, Canada, Vol. 2, AP. 18-2, pp. 696-699 June 1980

The Time-Domain Characteristics of a Traveling-Wave Linear Antenna with Linear and Nonlinear Parallel Loads

IEEE Trans. Antennas Propagat., Vol. AP-28, No. 2, pp. 267-276 March 1980

SREENIVASIAH, I .; CHANG, D. C.; MA, M. T.

Characterization of Electrically Small Radiating Sources by Tests Inside a Transmission Line Cell

NBS Tech. Note 1017

March 1980

HILL, D. A.; WAIT, J.R.

Propagation Along a Coaxial Cable with a Helical Shield

IEEE Trans. Microwave Theory Tech., Vol. MTT-28, No. 2, pp. $84-89$

February 1980

RANDA, M.

Analytical and Numerical Techniques for Analyzing an Electrically Short Dipole with a Nonlinear Load

IEEE Trans. Antennas Propagat., Vol. AP-28, No. 1, pp. 71-78 January 1980

Transients in Resistively Loaded Antennas and Their Comparison with Conical Antennas and a TEM Horn

IEEE Trans. Antennas Propagat., Vol. AP-28, No. 1, pp. 132-136 January 1980

CRAWFORD, M. L.; WORKMAN, J. I.

Predicting Free-Space Radiated Emissions from Electronic Equipment Using TEM Cell and Open-Field Site Measurements

Record, 1980 Intl. Electromagn. Compat. Symp. 1980

RANDA, M.; RIES, F. Z.; BELSHER, D. R.

A Broadband Isotropic, Real-Time, Electric Field Sensor (BIRES) Using

Resistively Loaded Dipoles

NBSIR 79-1622

December 1979 
KANDA, M.

The Effects of Resistive Loading on TEM Horns

NBSIR 79-1601

August 1979

ARTHUR, M. G.

Impulse spectral Intensity -- What is it?

Proc., 1978 Electromagn. Interference Workshop

NBS SP 551

July 1979

TIPPET, J. C.; CHANG, D. C.

Dispersion and Attenuation Characteristics of Modes in a TEM Cell with a Lossy Dielectric Slab

NBSIR 79-1615

July 1979

CRAWFORD, M. I.

Comparison and Selection of Techniques for Measuring EM Radiated

Emissions and Susceptibility of Large Equipment

Proc., 1979 Symp. and Technical Exhib. on Electromagn. Compat.

(3rd), 1-3 May 1979, Rotterdam, Netherlands, pp. 115-122 May 1979

MA, M. T.

A Theoretical Study of Unbalanced Ground Effects on Receiving Dipoles NBSIR 79-1605

May 1979

SREENIVASIAH, I.; CHANG, D. C.

Variational Expression for the Scattering Matrix of a Coaxial Iine step

Discontinuity and its Application to an over Moded Coaxial TEM Cell NBSIR 79-1606 May 1979

CRAWFORD, M. I.; WORKMAN, J. I.

Using a TEM Cell for EMC Measurements of Electronic Equipment NBS Tech. Note 1013 April 1979

DECKER, W. F.; CRAWFORD, M. I.; WILSON, W. A.

Construction of a Large Transverse Electromagnetic cell

NBS Tech. Note 1011

February 1979

RANDA, $\mathbf{M}$.

The Time Domain Characteristics of a Traveling Wave Linear Antenna with Linear and Non-Linear Loads

NBSIR 78-892(R)

February 1979

The Characteristics of a Traveling-Wave, Linear Antenna with a Nonlinear Load

Dig. Abstract, 1979 IEEE Ant. Propagat. Soc. Symp. January 1979 
RANDA, M. (cont.)

Analytical and Numerical Techniques for Analyzing an Electrically Short

Dipole with a Nonlinear Load

NBSIR 78-898

November 1978

LAWTON, R. A.; ONDREJKA, A. R.

Antennas and the Associated Time Domain Range for the Measurement of

Impulsive Fields

NBS Tech. Note 1008

November 1978

TIPPET, J. C.; CHANG, D. C.

Characteristic Impedance of a Rectangular Coaxial Line With offset Inner Conductor

IEEE Trans. Microwave Theory Technol., Vol. MTT-26, No. 11,

Pp. $876-883$

November 1978

CRAWFORD, M. I.; WORKMAN, J. L.; THOMAS, C. I.

Expanding the Bandwidth of TEM Cells for EMC Measurements

IEEE Trans. Electromagn. Compat., Vol. EMC-20, No. 3, pp. 368-375 August 1978

ANDREWS, J. R .; BAIDWIN, E. E.

SHF Impulse Generator

NBSIR 78-888

June 1978

CRAWFORD, M. I.; WORKMAN, J. I.

Asymmetric Versus symmetric TEM Cells for EMI Measurements

Proc., 1978 IEEE Intl. Symp. on Electromagn. Compat.,

20-22 June 1978, Atlanta, GA, pp. 204-210

June 1978

ANDREWS, J. R.; BALDWIN, E. E.

UHF Impulse Generator

NBSIR 78-880

April 1978

RANDA, M.

Transients in Resistively Loaded Antennas and Their Comparison with Conical Antennas and TEM Horns

NBSIR 78-876

March 1978

TIPPET, J. C.; CHANG, D. C.

Higher Order Modes in Rectangular Coaxial Line with Infinitely Thin

Inner Conductor

NBSIR 78-873

March 1978

ADAMS, J. W.; TAGGART, H. E.

Electromagnetic Compatibility Evaluation of seven Electronic Brake

systems

NBSIR 78-872

February 1978

MILLER, C. R. S.

EMI - A Problem of Growing concern

Report of the 63rd Natl. Conf. on Weights and Measures

NBS SP 532

1978 
IARSEN, E. B.; SHAFER, J. F.

Surveys of Electromagnetic Field Intensities Near Representative Higher-Power FAA Transmitting Antennas

FAA-RD -77

December 1977

RANDA, M.

The Characteristics of Broadband, Isotropic Electrical Field and Magnetic Field Probes

NBSIR 77-868

November 1977

ANDREWS, J. R.; ARTHUR, M. G.

Spectrum Amplitude--Definition, Generation and Measurement NBS Tech. Note 699

October 1977

CRAWFORD, M. L.; WORKMAN, J. I.; THOMAS, C. L.

Generation of EM Susceptibility Test Fields Using a Large

Absorber-Loaded TEM Cell

IEEE Trans. Instrum. Meas., Vol. IM-26, No. 3, pp. 336-343 September 1977

ADAMS, J. W.; KANDA, M.; SHAFER, J. F.; WU, Y.

Near-Field Electric Field Strength Levels of EM Environments Applicable to Automotive systems

Proc., 1977 IEEE Intl. Symp. on Electromagn. Compat. 2-4 August

1977, seattle, WA, pp. 336-343

August 1977

CRAWFORD, M. L.; THOMAS, C. I.

Converting a Rectangular shielded Enclosure into a TEM Transmission Cell for EMI Measurements

Proc., 1977 IEEE Intl. Symp. on Electromagn. Compat., 2-4 August

1977, Seattle, WA, pp. 1-6

August 1977

KANDA, $M$.

A Relatively short Cylindrical Broadband Antenna with Tapered Resistive

Loading for Picosecond Pulse Measurements

NBSIR 77-861

August 1977

A Broadband Antenna with Tapered Resistive Loading for EMI Measurements Proc., 1977 IEEE Intl. Symp. on Electromagn. Compat., 2-4 August 1977, Seattle, WA, pp. 13-18 August 1977

BENSEMA, W. D.

Amplitude, Time, and Frequency Statistics of Quasi-Impulsive Noise

Proc., 1977 Electromagn. Compat. Symp., 18-30 June 1977, Montreux, Switzerland, pp. 347-352

June 1977

RANDA, $\mathbf{M}$.

The Characteristics of a Relatively Short Broadband Linear Antenna with Tapered Resistive Loading

Proc., Intl. Symp. Ant. Propagat. Soc., 20-22 June 1977, pp. 230233

June 1977 
BENSEMA, W. D.

A Noise Spectrum Measurement System Using the Fast Fourier Transform IEEE Trans. Electromagn. Compat., Vol. EMC-19, No. 2, pp. 37-43

May 1977

FLANIGAN, W. F. Jr.; BOWMAN, R. R.; LOWELL, W. R. Nonmetaliic Electrode System for Recording EEG and ECG in Electromagnetic Fields

Physiology and Behavior, Vol. 18, No. 3, pp. 531-533 1977

RANDA, M.

A Relatively Short Cylindrical Broadband Antenna with Tapered Resistive Loading for Picosecond Pulse Measurements

IEEE Trans. Antennas Propagat., Vol. AP-26, No. 3, pp. 439-447 1977

ANDREWS, J. $R$.

Impulse Generator Spectrum Amplitude Measurement Techniques

IEEE Trans. Instrum. Meas., Vol. IM-25, No. 4, pp. 380-384

December 1976

GREENE, F . M.

Measurement of RF Power-Absorption in Biological Specimens (10 to

$100 \mathrm{MHz}$ )

NBS Tech. Note 687

November 1976

LARSEN, E. B.; ANDREWS, J. R.; BALDWIN, E. E.

Sensitive Isotropic Antenna with Fiber-Optic Link to a Conventional

Receiver

NBSIR 75-819

September 1976

TIPPET, J. C.; CHANG, D. C.

A New Approximation for the Capacitance of a Rectangular, Coaxial, Strip Transmission Iine

IEEE Trans. Microwave Theory Tech., pp. 602-604

September 1976

CRAWFORD, M. L.

Improved Techniques and Instrumentation for EMC Measurements

IEEE 1976 Intl. Symp. on Electromagn. Compat., 13-15 July 1976, Washington, DC

July 1976

LARSEN, E. B.; ANDREWS, J. R.

Using Fiber Optics in A Broadband, Sensitive, Isotropic Antenna -

$15 \mathrm{KHz}$ to $150 \mathrm{MHz}$

Proc., 1976 IEEE Intl. Symp. on Electromagn. Compat.,

13-15 July 1976, Washington, DC July 1976

CRAWFORD, M. L.

Experimental Evaluation of the Radiation Characteristics of Dipole Sources Enclosed in a TEM Transmission Cell

Proc., 1976 Conf. on Precision Electromagn. Meas., 28 June-1 July

1976, Boulder, CO, pp. 57-59

June 1976 
REEVE, G. R.

A Standard Percent Modulation Measurement System

Dig. 1976 Conf. Prec. Electromagn. Meas., 28 June-1 July 1976, Boulder, CO, pp. 165-166 June 1976

TIPPET, J. C. ; CHANG, D. C.; CRAWFORD, M. L.

An Analytical and Experimental Determination of the Cutoff Frequencies of Higher-Order TE Modes in a TEM Cell NBSIR 76-841 June 1976

BOWMAN, $R$. $R$.

Calibration Techniques for Electromagnetic Hazard Meters: $500 \mathrm{MHz}$

To $20 \mathrm{GHz}$

NBSIR 75-805

April 1976

ADAMS, J. W.; CRAWFORD, M. L.; SHAFER, J. F.

Electromagnetic Interference (EMI) Measurements for Automotive Applications

Proc., 1976 SAE Automotive Congress Conf., 23-27 February 1976, Detroit, MI, pp. 1-6 February 1976

BOWMAN, R. R.

A Probe for Measuring Temperature in Radio-Frequency-Heated Material

IEEE Trans. Microwave Theory Technol., Vol. MTT-24,

No. 1 , pp. 43-45

January 1976

TIPPET, J. C.; ChANG, D. C.

Radiation Characteristics of Dipole Sources Located Inside a

Rectangular, Coaxial Transmission Line

NBSIR 75-829

January 1976

CRAWFORD, M. L.

Techniques for Measurement of Electromagnetic Radiation and

Susceptibility of Electronic Equipment

Proc., 1975 Symp. and Technical Exhib. on Electromagn. Compat.

(1st), 20-22 May 1975, Montreux, Switzerland, pp. 38-44 May 1975

RANDA, M.; ADAMS, J. W.

Amplitude Statistics of Electromagnetic Noise in Coal Mines

Dig., Intl. Union of Radio Sci. (Union Radio Scientifique

Internationale), IEEE Trans. Antennas Propagat.

May 1975

BELSHER, D. R.

Development of Near-Field Electric Energy Density Meter Model EDM-2 Department of Health, Education, and Welfare, Publ. No. (NIOSH) 75-140

March 1975

CRAWFORD, M. L.

Generation of Standard EM Fields for Calibration of Power Density Meters $20 \mathrm{kHz}$ to $1000 \mathrm{MHz}$

NBSIR 75-804

January 1975 
GREENE, F. M.

Development of Electric and Magnetic Near-Field Probes

NBS Tech. Note 658

January 1975

FITZGERRELI, R. G.

Experimental RF Field Polarization Meter

FAA-RD $-74-188$

1975

CRAWFORD, M. I.

Generation of Standard EM Fields Using TEM Transmission Cells

IEEE Trans. Electromagn. Compat., Vol. EMC-16, No. 4, pp. 189-195 November 1974

SCOTT, W. W., Jr.; ADAMS, J. W.; BENSEMA, W. D.; DOBROSRI, H.

Electromagnetic Noise in Lucky Friday Mine NBSIR 74-391

1974

KANDA, M.

Time and Amplitude Statistics for Electromagnetic Noise in Mines NBSIR 74-378 July 1974

NEWELI, A. C.; CRAWFORD, M. I.

Planar Near-Field Measurements on High Performance Array Antennas NBSIR 74-380 July 1974

ADAMS, J. W.; BENSEMA, W. D.; RANDA, M. Electromagnetic Noise in Grace Mine NBSIR 74-388

June 1974

ADAMS, J.W.; BENSEMA, W. D.; TOMOEDA, N. C. Surface Magnetic Field Noise Measurements at Geneva Mine NBSIR 74-369

June 1974

BENSEMA, W. D.; RANDA, M.; ADAMS, J. W.

Electromagnetic Noise in Itmann Mine NBSIR 74-390

June 1974

CRAWFORD, M. L.

Evaluation of Reflectivity Level of Anechoic Chambers Using Isotropic, 3-Dimensional Probing

Proc., 1974 Intl. IEEE Ant. Propagat. Soc. Symp., 10-12 June 1974, Atlanta, GA, pp. 28-34 June 1974

HUDSON, P. A.; MELQUIST, D. G.; ONDREJKA, A. R.; WERNER, P. E. Completion of the Program to Evaluate/Improve Instrumentation and Test Methods for Electroexplosive Device Safety Qualification NBSIR 74-379 June 1974

RANDA, M.; ADAMS, J. W.; BENSEMA, W. D. Electromagnetic Noise in McElroy Mine NBSIR 74-389

June 1974 
SIMPSON, P. A.

Broadband Pulsed/CW Calibration Signal Standard for Field Intensity

Meter (FIM) Receivers

NBSIR 74-371

June 1974

ARTHUR, M. G.

Impulse spectral Intensity -- What is it?

NBSIR 74-365

May 1974

GREENE, F. $M$.

Development and Construction of an Electromagnetic Near-Field

synthesizer

NBS Tech. Note 652

May 1974

BENSEMA, W. D.; RANDA, M.; ADAMS, J.W.

Electromagnetic Noise in Robena No. 4 Coal Mine NBS Tech. Note 654

April 1974

ANDREWS, J. R.

Picosecond Pulse Generators Using Microminiature Mercury Switches NBSIR 74-377

1974

BAIRD, R. C.

Methods of Calibrating Microwave Hazard Meters

Proc., 1973 Intl. Symp on Biological Effects and Health Hazards of Microwave Radiation, 15-18 October 1973, Warsaw, Poland, pp. 228-236

October 1973

BOWMAN, $\mathbf{R}$. $\mathbf{R}$.

Some Recent Developments in the Characterization and Measurement of Hazardous Electromagnetic Fields

Proc., 1973 Intl. Symp. on Biological Effects and Health Hazards

of Microwave Radiation, 15-18 October 1973, Warsaw, Poland,

pp. 217-227

October 1973

REEVE, G. R.

Calibration of Impulse Noise Generators

NBSIR 73-343

1973

LARSEN, E. B.

Calibration of Radio Receivers to Measure Broadband Interference NBSIR 73-335

September 1973 
RANDA, M.; ADAMS, J.W.

Amplitude Statistics of Electromagnetic Noise in Coal Mines Proc., 1973 Thru-the-Earth Electromagn. Workshop, 15-17 August 1973, Colorado School of Mines, Golden, CO, pp. 156-160 August 1973

HUDSON, P. A.; MELQUIST, D. G .; ONDREJKA, A. R.; WERNER, P. E. A Program to Evaluate/Improve Instrumentation and Test Methods for Electroexplosive Device Safety Qualification NBSIR 73-323

June 1973

NAHMAN, N. S.

Miniature Superconductive Coaxial Transmission Iines

Proc., IEEE, Vol. 61, pp. 76-79

January 1973

BENSEMA, W. D.

Coal Mine Magnetic-Field Noise Measurements

Proc., 1972 W. Virginia Conf. on Coal Mine Electrotechnology (1st), 2-4 August 1972, West Virginia Univ., Morgantown, WV, pp. XII-1 through XII-12 August 1972

ADAMS, J. W.; TAGGART, H. E.; SPAULDING, A. D. Survey Report of the U.S. Bureau of Mines Electromagnetic Noise Measurement Program

NBS Rept. 10723

November 1971

WACKER, P. F.; BOWMAN, R. R.

Quantifying Hazardous Electromagnetic Fields: Scientific Basis and Practical Considerations

IEEE Trans. Microwave Theory Tech., Vol. MTT-19, No. 22 February 1971

BOWMAN, R. R。

Quantifying Hazardous Electromagnetic Microwave Fields: Practical Considerations

Proc., 1969 symp. Biological Effects and Health Implications of Microwave Radiation, 17-19 September 1969, Richmond, VA., BRH/DBE 70-2, U.S. Dept. of Health, Education, and Welfare, Rockville, MD, pp. 204-209

June 1970

WACKER, P. F.

Quantifying Hazardous Microwave Fields: Analysis

Proc., 1969 symp. on Biological Effects and Health Implications of Microwave Radiation, 17-19 September 1969, Richmond, VA, BRH/DBE 70-2, U.S. Dept. of Health Education, and Welfare, Rockville, MD pp. 197-203 June 1970

LAWTON, R. A.

A New Standard for Electric Field Strength

IEEE Trans. Instrum. Meas., Vol. IM-19, pp. 45-51 February 1970 


\section{MICROWAVE METROLOGY}

JARGON, J. A.; REBULDELA, G. Measurement Service for High-Power $\mathrm{CW}$ Wattmeters at the National Institute of Standards and Technology Proc., Meas. Sci. Conf., 20-22 January 1993, Anaheim, CA, Session 5-B (pages not numbered) January 1993

SHERWOOD, G. V.

Dimensional Characterization of Precision Coaxial Transmission Line Standards

Proc., Meas. Sci. Conf., 20-22 January 1993, Anaheim, CA, pp. 1-10 January 1993

MARRS, R. B.; WIILIAMS, D. F.

Interconnection transmission Line Parameter Characterization

Dig., 40th Auto. RF Tech. Group Conf., 3-4 December 1992, Orlando, FL, pp. 88-95

December 1992

WALRER, D. R.; WILLIAMS, D. F.; MORGAN, J. M.

Planar Resistors for Probe station Calibration Dig., 40th Auto. RF Tech. Group Conf., 3-4 December 1992, Orlando, FL, pp. 1-9 December 1992

WILIIAMS, D. F.; MARRS, R. B.

Calibrating On-Wafer Probes to the Probe Tips

Dig., 40th Auto. RF Tech. Group Conf., 3-4 December 1992, Orlando, FL, pp. 136-143

December 1992

REBULDELA, G :; JARGON, J. A.

High Power CW Wattmeter Calibration at NIST

$\mathrm{J}$. of Res of the Nat'l Inst of Stds and Tech, Vol., No., pp.673687

November - December 1992

WIILIAMS, D. F.; MARKS, R. B.; WALRER, D. R.; CLAGUE, F. R .

Wafer Probe Transducer Efficiency

IEEE Microwave and Guided Waves Lett., Vol. 2, No. 10, pp. 388-390 October 1992

MARKS, R. B.; WIILIAMS, D. F.

A General Waveguide Circuit Theory

NIST JRES, Vol. 97, No. 5, pp. 533-562

september - October 1992

HUANG, D. X.; REBULDELA, G.

High-Frequency Response of Micropotentiometers

Proc., NCSL, 2-6 August 1992, Washington, DC, pp. 547-555

August 1992

MARKS, R. B.; WILLIAMS, D. F.

Accurate Experimental Characterization of Interconnects: A Discussion of "Experimental Electrical Characterization of Interconnects and Discontinuities in High-Speed Digital systems"

IEEE Trans. on Components, Hybrids, and Manufacturing Technology,

Vol. 15, No. 4, pp. 601-604

August 1992 
WILLIAMS, D. F.; MARKS, R. B.

Comments on "Characterization of Resistive Transmission Lines by ShortPulse Propagation"

IEEE Microwave and Guided Waves Lett., Vol. 2, No. 8, p. 346 August 1992

CLAGUE, F. R.

A New Coaxial Microwave Microcalorimeter Evaluation Technique

Dig., 1992 Conf. on Prec. Electromagn. Meas., 9-12 June 1992,

Paris, France, pp. 387-388

June 1992

MARKS, R. B.; WILIIAMS, D. F.

Traceability for On-Wafer MMIC Measurements

Dig., 1992 Conf. on Prec. Electromagn. Meas., 9-12 June 1992,

Paris, France, pp. 91-92

June 1992

REBULDELA, G.; JARGON, J.A.

High Power CW Wattmeter Calibration at NIST

Dig., 1992 Conf. on Prec. Electromagn. Meas., 9-12 June 1992,

Paris, France, pp. 381-382

June 1992

WILLIAMS, D. F.; MARKS, R. B.

Verification of Scattering Parameter Measurements

Dig., 1992 Conf. on Prec. Electromagn. Meas., 9-12 June 1992,

Paris, France, pp. 371-372

June 1992

ESTIN, A. J.; JUROSHEK, J. R.; MARKS, R. B.; CLAGUE, F. R.; ALLEN, J. W. Basic RF and Microwave Measurements: A Review of Selected Programs Metrologia, Vol. 29, No. 2, pp. 135-151 May 1992

MARRS, R. B .

Comments on "Rapid Pulsed Microwave Propagation"

IEEE Microwave and Guided Wave Lett., Vol. 2, No. 5, p. 204 May 1992

WILLIAMS, D. F.; MARKS, R. B.

Frequency-Dependent Transmission Line Parameters

Dig., IEEE Topical Meeting on Electrical Performance of Electronic Packaging, 22-24 April 1992, Tucson, AZ, pp. 125-127 April 1992

FREE, G. M.; JONES, R. N.

Calibration Service for Low-Loss, Three-Terminal Capacitance standards at $100 \mathrm{kHz}$ and $1 \mathrm{MHz}$

NIST Tech. Note 1348

February 1992

FURLOW, R.; SHIMODA, R. Y.; WILIIAMS, D. F.; MARRS, R. B.; GUPTA, K. C. Benchmark for the Verification of Microwave CAD Software

Proc., 38th Auto. RF Tech. Group Conf., 5-6 December 1991, San

Diego, CA, pp. 97-106

December 1991

MARKS, R. B.; WILLIAMS, D. F.

Reciprocity Relations for On-Wafer Power Measurements

Proc., 38th Auto. RF Tech. Group Conf., 5-6 December 1991, San

Diego, CA,pp. 82-89

December 1991 
WIILIAMS, D. F.; MARKS, R. B.; DAVIDSON, A.

Comparison of On-Wafer Calibrations

Proc., 38th Auto. RF Tech. Group Conf., 5-6 December 1991, San

Diego, CA, pp. 68-81

December 1991

MARKS, R. B.; WIILIAMS, D. F.

Transmission Line Capacitance Measurement

IEEE Microwave and Guided Wave Lett., Vol. 1, No. 9, pp. 243-245 september 1991

REEVE, G. R.

Millimeter Wave Metrology at the National Institute of standards and Technology

PrOC., 1991 NCSI Workshop and Symp., 18-22 August 1991,

Albuquerque, NM, pp. 183-195

August 1991

MARKS, R. B.

A Multi-Line Method of Network Analyzer Calibration

IEEE Trans. Microwave Theory Tech., Vol. MTT-39, No. 7, p. 12051215

July 1991

MARRS, R. B.; WIILIAMS, D. F.

Characteristic Impedance Determination Using Propagation Constant Measurement

IEEE Microwave and Guided Wave Lett., Vol. 1, No. 6, p. 141-143 June 1991

JUDISH, R. M.; BURNS, J. G.

Measurement Program Compares Automatic Vector Analyzers

Microwaves and RF, Pp. 203-206

May 1991

WIILIAMS, D. F.; MARRS, R. B.; PHILIIPS, R. R.

Translate LRI and LRM Calibrations

Microwaves and RF, $\mathrm{pp}$. 78-84

February 1991

SEERWOOD, G. V.

Air Gage Size Measurement of Microwave Standards

Proc., 1991 Measurement Science Conf. 31 Jan. - 1 Feb. 1991, Anaheim, $\mathrm{CA}, \mathrm{pp} .1-10$

January 1991

REEVE, G. R.; MARKS, R. B.; BLACKBURN, D.

Microwave Monolithic Integrated Circuit-Related Metrology at the National Institute of standards and Technology

IEEE Trans. Instrum. Meas., Vol. IM-39, No. 6, pp. 958-961 December 1990

CLAGUE, F. R.

Power Measurement System for $1 \mathrm{~mW}$ at $1 \mathrm{GHz}$

NIST Tech. Note 1345

November 1990 
MARKS, R. B.

Multi-Line Calibration for MMIC Measurement

Dig., 36th Auto. RF Tech. Group Conf., 29-30 November 1990, Monterey, $\mathrm{CA}$,

pp. 47-55

November 1990

PHILIIPS, R. R.; WILIIAMS, D. F.

MMIC Package Characterization with Active Loads

Dig., 36th Auto. RF Tech. Group Conf., 29-30 November 1990, Monterey, CA,

pp. 64-72

November 1990

WIILIAMS, D. F.; MARKS, R. B.

The Interpretation and Use of $\mathrm{S}$-Parameters in Lossy Lines Dig., 36th Auto. RF Tech. Group Conf., 29-30 November 1990, Monterey, CA,

pp. 84-89

November 1990

WIILIAMS, D. F.; MARRS, R. B.; PHILIIPS, R. R.; MIERS, T. Progress Toward MMIC On-Wafer Standards

Dig., 36th Auto. RF Tech. Group Conf., 29-30 November 1990, Monterey, $\mathrm{CA}$,

pp. 73-83

November 1990

HOLT, D. R.

Determination of scattering parameters with Respect to the

Characteristic Impedance of Precision Coaxial Air-Line Standards

Dig., Conf. Prec. Electromagn. Meas., 11-14 June 1990, Ottawa,

Canada, pp. 282-283

June 1990

MARRS, R. B.

Comments on "Improved Calibration and Measurement of the scattering Parameters of Microwave Integrated Circuits"

IEEE Trans. Microwave Theory Tech., Vol. MTT-38, No. 4, p.453 April 1990

REEVE, G. R.; MARKS, R. B.; BLACKBURN, D.

MMIC Related Metrology at the National Institute of Standards and Technology

Record, Instrum. Meas. Tech. (IMTC) Conf.; 13-15 February 1990;

San Jose, CA; Pp. 196-199

February 1990

MARKS, R. B.; PHILLIPS, R. R.

Wafer-Level ANA Calibrations at NIST

Dig., 34th Auto. RF Tech. Group Conf., 30 November - 1 December

1989 , Ft. Lauderdale, FL, pp. 11-25

December 1989 
WILIIAMS, D. F.

Development of On-Wafer Microwave Standards at NIST

Dig., 34th Auto. RF Tech. Group Conf., 30 November - 1 December

1989, Ft. Lauderdale, FL, pp. 5-10

December 1989

ADAIR, R. T.; IIVINGSTON, E. M.

Coaxial "Intrinsic" Impedance Standards

NIST Tech. Note 1333

october 1989

JUROSHER, J.R.; HOER, C. A.; RAISER, R. R. F.

Calibrating Network Analyzers with Imperfect Test ports

IEEE Trans. Instrum. Meas., Vol. 38, No. 4, pp. 898-901 August 1989

HOER, C. A.

Systematic Errors in Power Measurements Made with a Dual Six Port ANA;

NIST Tech. Note 1332

July 1989

WEIL, C. M.; MARLER, F. E.; MAJOR, J. R.; WEIDMAN, M. P.;

RUSSELL, D. H.

Dual Six-Port Reflectometer Systems Using Waveguide in the Frequency

Range 18-50 $\mathrm{GHz}$.

Dig., 33rd Auto. RF Tech. Group Conf., 15-16 June 1989, Long

Beach, CA, pp. 76-87

June 1989

HOLT, D. R.

Scattering Parameters Representing Imperfections in Precision Coaxial

Air Lines

NIST JRES, Vol. 94 , No. 2, pp. 117-133

March-April 1989

CLAGUE, F. R.

The NIST Automated Coaxial Microwave Power standard

Proc., 1989 Measurement Science Conf., 26-27 January 1989,

Anaheim, $\mathrm{CA}$, pp. 1C-1 through 1C-14

January 1989

LIVINGSTON, E. M.; ADAIR, R. T.

Performance Evaluation of Radiofrequency, Microwave and Millimeter Wave Power Meters

NBS Tech. Note 1310

December 1988

CoUnas, G. J.; YATES, B. C.

Measurement of Adapter Loss, Mismatch, and Efficiency Using the Dual

Six-Port

NBSIR 88-3095

July 1988 
HOER, C. A.

An Equivalent Circuit for Imperfect Transmission Line Connectors

Dig., Conf. Prec. Electromagn. Meas., 7-10 June 1988, Tsukuba Science City, Japan, pp. 264-265 June 1988

HOLT, D. R.

Determination of Scattering Parameters from Precision Coaxial Air-Line Standards

Dig., Conf. Prec. Electromagn. Meas., 7-10 June 1988, Tsukuba Science City, Japan, p. 263 June 1988

ADAIR, R. T.; RUSSELI, D. H.

A Calibration Service for $30 \mathrm{MHz}$ Attenuation and Phase Shift NIST SP250-32

April 1988

ENGEN, G. F.

On-Line Accuracy Assessment for the Dual Six-Port ANA: Background and Theory

IEEE Trans. Instrum. Meas., Vol. IM-36, No. 2, pp. 501-506 June 1987

ADAIR, R. T.; REEVE, G. R.; GATTERER, L. E.

The Expanding Need for Microwave and Millimeter Wave Calibration

Services

NCSL Newsletter, Vol. 27, No. 1, pp. 21-31 January 1987

LARSEN, N. T.

Microwave Power Standards at the National Bureau of Standards

1987 NCSL Workshop and Symp., 12-16 July 1987, Denver, CO, pp. 34-1 through 34-7

July 1987

HOER, C. A.

On-Line Accuracy Assessment for the Dual Six-Port ANA; Treatment of Systematic Errors

IEEE Trans. on Instrum. and Meas., Vol. IM-36, No. 2, pp. 514-519 June 1987

Some Questions and Answers Concerning Air Lines as Impedance Standards Dig., 29th Auto. RF Tech. Group Conf., 12-13 June 1987, Las Vegas, NV,

pp. 161-173

June 1987

JUDISH, R. M.; ENGEN, G. F.

On-Line Accuracy Assessment for the Dual Six-Port ANA: Statistical Methods for Random Errors

IEEE Trans. Instrum. Meas., Vol. IM-36, No. 2, pp. 507-513 June 1987 


\section{Microwave Metrology (cont.)}

JUROSHER, J . R.

On-Line Accuracy Assessment for the Dual Six-Port ANA: Experimental Results

IEEE Trans. Instrum. Meas., Vol. IM-36, No. 2, pp. 520-523 June 1987

A Study of Measurement of Connector Repeatability Using Highly

Reflecting Loads

IEEE Trans. Microwave Theory Tech., Vol. MTT-35, No. 4, pp. 457-460

April 1987

SAULSBERY, L. F.; ADAIR, R. T.

ANA Measurement Results on the Auto. RF Tech. Group Traveling Experiment Conf. Dig., 28th Auto. RF Tech. Group Conf., 4-5 December 1986, St. Petersburg Beach, FL, Pp. 65-78 January 1987

CLAGUE, F. R.; IARSEN, N. T.

A Transient Response Error in Microwave Power Meters Using Thermistor Detectors

Dig., 28th Auto. RF Tech. Group Conf., 4-5 December 1986, st.

Petersburg Beach, FI, pp. 79-89

December 1986

ENGEN, G. F.

On-Line Accuracy Assessment for the Dual Six-Port ANA: Background and Theory

Dig., 1986 Conf. Prec. Electromagn. Meas., 23-27 June 1986, NBS/Gaithersburg, MD,

p. 236

June 1986

In Search of a More Realistic Accuracy Statement for Microwave Metrology Dig., 27th Auto. RF Techniques Group, 5-6 June 1986, Baltimore, MD, pp. 181-183

June 1986 
HOER, C. A.

On-Iine Accuracy Assessment for the Dual Six-Port ANA; Treatment of Systematic Errors

Dig., 1986 Conf. Prec. Electromagn. Meas., 23-27 June 1986, NBS, Gaithersburg, MD, pp. 238-239 June 1986

HOER, C. A.; ENGEN, G. F.

Calibrating a Dual Six-Port or Four-Port for Measuring Two-Ports with Any Connectors

Dig., 1986 IEEE Microwave Theory Tech. Symp., 2-4 June 1986, Baltimore, MD, pp. 665-668 June 1986

On-Line Accuracy Assessment for the Dual Six-Port ANA: Extension to Non-Mating Connectors

Dig., 1986 Conf. Prec. Electromagn. Meas., 23-27 June 1986, NBS, Gaithersburg, $M D$ pp. 241-242 June 1986

JUDISH, R. M.

On-Line Accuracy Assessment for the Dual Six-Port ANA: Statistical

Methods for Random Errors

Dig., 1986 Conf. Prec. Electromagn. Meas., 23-27 June 1986, NBS, Gaithersburg, $M D$, P. 237 June 1986

JUROSHER, J . R.

On-Iine Accuracy Assessment for the Dual Six-Port ANA: Experimental Results

Dig., 1986 Conf. Prec. Electromagn. Meas., 23-27 June 1986, NBS, Gaithersburg, MD, p. 240 June 1986

ADAIR, R. T.; REEVE, G. R.; GATTERER, I. E.

Millimeter Wave Standards: An Emerging Need

Proc., IEEE Instrum. and Meas. Techn. Conf., 25-27 March 1986,

Boulder, CO, Vol. IM-35, No. 4, pp. 376-382 March 1986

GINLEY, R. A.; ALLRED, C. M .

1.25 $\mathrm{MHz}$ Attenuation Measurement system

Proc., IEEE Instrum. and Meas. Techn. Conf., 25-27 March 1986, Boulder, CO, Vol. IM-35, No. 4, pp. 463-466 March 1986

MAJOR, J.R.; IIVINGSTON, E. M.; ADAIR, R. T.

Automatic Frequency Response of Frequency Modulated Generators Using the Bessel Null Method

NBS Tech. Note 1093

March 1986

JUISH, R. M.

Quality Control of Measurements--Measurement Assurance

Proc., IEEE Spec. Issue on Radio Meas. Methods and standards,

Vol. 74, No. 1 , pp. 23-25

January 1986 
SLADER, M. J.; JESCH, R. L.

Standardization of Coaxial Connections in the IEC Proc., IEEE Spec. Issue on Radio Meas. Methods and standards, pp. $14-18$ January 1986

HOER, C. A.

Multiport Network Analyzers

MCGraw-Hill Yearbook of Science and Technol., pp. 289-292 1986

HOLT, D. R.; HOER, C. A.

Estimation of True Power Ratios in Six-port Network Analyzers Using Diode Detectors

IEEE Trans. Instrum. Meas., Vol. IM-34, No. 4, pp. 558-563 December 1985

WEIDMAN, M. P.

Finline Diode Six-Port: Fundamentals and Design Information NBS Tech. Note 1090 December 1985

SEQUEIRA, H. B.; YATES, B. C.

Approach for Evaluating Effects of Wall Losses on Quarter-Wave Losses on Quarter-Wave Short-Circuit Impedance Standards IEEE Trans. Microwave Theory Tech., Vol. MTT-33, No. 11, pp. 1106-1109 November 1985

JUROSHER, J. R.; HOER, C. A.

A Technique for Extending the Dynamic Range of the Dual Six-Port Network Analyzer IEEE Trans. Microwave Theory Tech., Vol. MTT-33, No. 6, pp. $453-459$ June 1985

MAJOR, J. R.; LIVINGSTON, E. M.; ADAIR, R. T. Automatic Frequency Response of Frequency Modulated Generators Using the Bessel Null Method Proc., 1985 Test and Measurement World Expo, 14-16 May 1985, San Jose, CA, pp. 78-100 May 1985

HOLT, D. R.; HOER, C. A.

Estimation of True Power Ratios in Six-port Network Analyzers Using Diode Detectors Proc., 1985 Instrum. and Meas. Technol. Conf., 20-22 March 1985, Tampa, FI, pp. 140-141 March 1985

REEVE, G. R.; MILLER, C. K. S. Current NBS Metrology Capabilities and Limitations at Millimeter Wave Frequencies

Proc., 1985 Meas. Science Conf., 17-18 January 1985, Santa Clara, CA, Pp. 296-314 January 1985 
MAJOR, J. R.; LIVINGSTON, E. M.; ADAIR, R. T. Automatic Frequency Response of Frequency Modulated Generators Using the Bessel Null Method

Dig., 24th Auto. RF Tech. Group Conf., 5-6 December 1984, Columbia, MD, pp. 131-153

December 1984

JUDISH, R. M.; JONES, R. N.

A Generalized Method for the Calibration of Four-Terminal-Pair Type

Digital Impedance Meters NBSIR 84-3016

August 1984

JUROSHER, J. R.; HOER, C. A.

A High-Power Automatic Network Analyzer for Measuring the RF Power

Absorbed by Biological samples in a TEM Cell

IEEE Trans. Microwave Theory Tech., Vol. MTT-32, No. 8, pp. 818-824

August 1984

A Dual Six-Port Network Analyzer Using Diode Detectors IEEE Trans. Microwave Theory Tech., Vol. MTT-32, No. 1, pp. 78-82

January 1984

KAMPER, R. A.; HOER, C. A.

Millimeter Wave Standards at the National Bureau of Standards (NBS)

Proc., The Intl. Soc. for Optical Engineering (SPIE),

23-24 August 1983, San Diego, CA, Vol. 423, pp. 144-146

August 1983

ENGEN, G. F.

Redundancy: A Monitor of Six-Port Performance

Dig., IEEE 1983 Colloquium on Advances in S-Parameter Measurement at Microwave Lengths, 23 May 1983, Savoy Place, London, England, No. 1983/53, pp. 4-1 through 4-2 May 1983

HOER, C. A.

Choosing Line Lengths for Calibrating Network Analyzers

IEEE, Trans. Microwave Theory Tech., Vol. MTT-31, No. 2, pp. 76-78 January 1983

RAMPER, R. A.

Current Trends in NBS Calibration Services

NCSL Newsletter, Vol. 22, No. 1, pp. 38-39

March 1982

HOER, C. A.

A High Power Dual Six-Port Automatic Network Analyzer Used in Determining Biological Effects of RF and Microwave Radiation

IEEE Trans. Microwave Theory Tech., Vol. MTT-29, Pp. 157-159 December 1981 
WEIDMAN, M. P.

WR-10 Single Six-Port Measurement System

NBSIR $81-1650$

September 1981

WEIDMAN, M. P.; HUDSON, P. A.

WR-10 Millimeter Wave Microcalorimeter

NBS Tech. Note 1044

June 1981

EBBESEN, H.; ENGEN, G. F.

Singularities in Calibration of Six-Port Network Analyzers

Dig., 1981 IEEE MTT-S Intl. Microwave Symp., p. 149 1981

ENGEN, G. F.

A Least Squares Solution for Use in Six-Port Measurement Technique IEEE Trans. Microwave Theory Tech., Vol. MTT-28, No. 12, pp. 1473-1477

December 1980

JONES, R. N.

Evaluation of Three-Terminal and Four-Terminal Pair Capacitors at High Frequencies

NBS Tech. Note 1024

September 1980

BILL, D. A.; WAIT, J.R.

Electromagnetic Theory of the Loosely Braided Coaxial Cable: Part II Numerical Results

IEEE Trans. Microwave Theory Tech., Vol. MTT-28, No. 4, pp. $326-331$

April 1980

Propagation Along a Coaxial Cable with a Helical Shield

IEEE Trans. Microwave Theory Tech., Vol. MTT-28, No. 2, pp. $84-89$

February 1980

REEVE, G. R.; ARTHUR, M. G.

A Standard for RF Modulation Factors

NBS Tech. Note 1016

September 1979

HOER, C. A.

Performance of a Dual Six-Port Automatic Network Analyzer

Proc., IEEE MTT-S 1979, Intl. Microwave Symp.: The World of Microwaves, 3 April-2 May 1979, Orlando, FL, IEEE Cat. No. 79CHI 439-9 MTT-S

April 1979 


\section{Microwave Metrology (cont.)}

JONES, R. N .

A Comparison of Centrifuge and Freezing Calorimeter Methods for Measuring Free Water in snow NBSIR 79-1604

April 1979

HOER, C. A.

Calibrating a Six-Port Reflectometer with Four Impedance Standards NBS Tech. Note 1012 March 1979

ENGEN, G. F.; HOER, C. A.

Through-Load-Delay: An Improved Technique for Calibrating the Dual

Six-Port Automatic Network Analyzer

Dig., Intl. Microwave Symp., Orlando, FL, p. 53 1979

DRIVER, L. D.; RIES, F. \& .; REBULDELA, G. NBS RF Voltage Comparator NBSIR 78-871

December 1978

ENGEN, G. F.

Calibrating the Six-Port Reflectometer by Means of Sliding Terminations IEEE Trans. Microwave Theory Tech., Vol. MTT-26, No. 12, pp. 951-957

December 1978

KAMPER, R. A.

Status of RF and Microwave Calibration Services at NBS

Natl. Conf. of Standards Laboratories Newsletter, Vol. 18, No. 3, p. 40

December 1978

HOER, C. A.

Calibrating Two Six-Port Reflectometers with Only One Impedance standard NBS Tech. Note 1004 June 1978

Calibrating Two Six-Port Reflectometers With an Unknown Length of Precision Transmission Line

Proc., 1978 IEEE MTT-S Intl. Microwave Symp., 27-29 June 1978,

Ottawa, Canada, pp. 176-178 June 1978

ENGEN, G. F.

The Six-Port Measurement Technique: A Status Report

Microwave J., Vol. 21, pp. 18, 21-22, 24, 84, 87, 89 May 1978

Advances in Microwave Measurement Science

Proc., IEEE, Vol. 66, pp. 374-384

April 1978

Instrumentation: Six Ports Simplify Network

Microwave Syst. News, pp. 54-55

January 1978 
ENGEN, G. F.; HOER, C. A.; SPECIALE, R. A.

The Application of "Through-Short-Delay" to the Calibration of the Dual Six-port

IEEE Intl. Microwave Symp. Dig., pp. 184-185

1978

KOMARER, E. I.

Advantages of the Six-port Reflectometer for RF/Microwave Power

Measurement in Operational Systems

Government Micro-Circuit Applications Conf. Dig. 1978

ENGEN, G. F.

An Improved Circuit for Implementing the Six-Port Technique of Microwave Measurements

IEEE Trans. Microwave Theory Tech., Vol. MTT-25, No. 12,

pp. 1080-1083

December 1977

HOER, C. A.

A Network Analyzer Incorporating Two 6-Port Reflectometers

IEEE Trans. Microwave Theory Tech., Vol. MIT-25, No. 12,

pp. 1070-1074

December 1977

JESCH, R. I.

Evaluation of Low-Loss/Low-Reflection Two-Port Devices or Adapters by Automated Measurement Techniques

NBS IR $78-870$

December 1977

ROMAREK, E. I.

Performance Characteristics of an Automated Broad-Band Bolometer Unit

Calibration System

IEEE Trans. Microwave Theory, Vol. MTT-25, No. 12, pp. 1122-1127

December 1977

WEIDMAN, M. P.

A Semi-Automated Six-Port for Measuring Millimeter Wave Power and Complex Reflection Coefficient IEEE Trans. Microwave Theory Technol., Vol. MTT-25, No. 12, pp. 1083-1085

December 1977

LARSEN, N. T.

NBS Type IV RF Power Meter operation and Maintenance

NBSIR 77-866

October 1977

ENGEN, G. F.

Design Considerations for Automatic Network Analyzers Based on the Six-Port Concept

Proc., IEE Euromeas 1977 Conf., 1-9 September 1977, Sussex,

England; London, England, pp. 110-111

september 1977 
ENGEN, G. F . (cont.)

The Six-Port Reflectometer: An Alternative Network Analyzer Proc., 1977 IEEE-MTT-S Intl. Microwave Symp., 21-23 June 1977, San Diego, CA, pp. 44-46 June 1977

An Improved Circuit for Implementing the Six-Port Technique of Microwave Measurements

1977 IEEE-MTT-S Intl. Microwave Symp., 21-23 June 1977,

San Diego, CA, pp. 53-55 June 1977

HOER, C. A.

A Microwave Network Analyzer Using Two 6-Port Reflectometers;

Proc., 1977 IEEE-MTT-S Intl. Microwave Symp.,

21-23 June 1977, San Diego, CA, pp. 47-49

June 1977

RAMPER, R. A.

The National Electromagnetic Measurement System NBSIR 75-936

June 1977

KOMARER, E. L.

An Application of the Six-Port Junction to Precision Measurement of Microwave One-Port Parameters

Proc., 1977 IEEE-MTT-S Intl. Microwave Symp., 21-23 June 1977, San

Diego, CA, pp. 56-57 June 1977

WEIDMAN, M. P.

A Semi-Automated Six-Port for Measuring Millimeter Wave Power and Complex Reflection Coefficient

Proc., 1977 IEEE-MTT-S Intl. Microwave Symp., 21-23 June 1977, San

Diego, CA, pp. 58-60 June 1977

ENGEN, G. F.

Determination of Microwave Phase and Amplitude from Power Measurements

IEEE Trans. Instrum. Meas., Vol. IM-25, pp. 414-418

December 1976

ESTIN, A. J.

Scattering Parameters of SMA Coaxial Connectors

IEEE Trans. Instrum. Meas., Vol. IM-25, No. 4, pp. 329-334 December 1976

JESCH, R. L.

Repeatability of SMA Coaxial Connectors

IEEE Trans. Instrum. Meas., Vol. IM-25, No. 4, pp. 314-320 December 1976

LARSEN, N. T.

A New Self-Balancing DC-Substitution RF Power Meter

IEEE Trans. Instrum. Meas., Vol. IM-25, No. 4, pp. 343-347 December 1976 
RIES, F. X.

An International Intercomparison of Voltage standards at $1 \mathrm{GHz}$ in Coaxial Transmission Line

IEEE Trans. Instrum. Meas., Vol. IM-25, No. 3, pp. 254-255 September 1976

ROE, K. C.; HOER, C. A.

A Microwave Vector voltmeter System NBSIR 76-844

August 1976

SIMPSON, P. A.; HUDSON, P. A.

Implementation of the Notch Technique as an RF Peak Pulse Power Standard NBS Tech. Note 682 July 1976

ENGEN, G. F.

Measurement of Complex Microwave Circuit Parameters Using Only Power Detectors

Proc., 1976 Conf. Prec. Electromagn. Meas.,

28 June-1 July 1976, Boulder, Co, p. 171 June 1976

HOER, C. A.; ROE, K. C.; ALIRED, C. M.

Measuring and Minimizing Diode Detector Nonlinearity

Proc., 1976 Conf. Prec. Electromagn. Meas., 28 June-1 July 1976, Boulder, Co, pp. 108-109 June 1976

KOMARER, E. I.

An Automated Broadband System for Measurement of One-Port Microwave Parameters Proc., 1976 Conf. Prec. Electromagn. Meas., 28 June-1 July 1976, Boulder, CO, pp. 167-170 June 1976

LARSEN, N. T.

A New Self-Balancing DC-Substitution RF Power Meter

Proc., 1976 Conf. Prec. Electromagn. Meas., 28 June-1 July 1976, Boulder, Co, pp. 203-205 June 1976

LITTLE, W. E.; YATES, B. C.

Reflection Coefficient Standards for Automated Network Analyzers

Proc., 1976 Conf. Prec. Electromagn. Meas., 28 June-1 July 1976, Boulder, Co, pp. 128-129 June 1976

IITTLE, W. E.; WAKEFIELD, J. P.; HEIM, L.; ALLRED, C. M.; ZAPF, T. L.

An NBS Developed Automatic Network Analyzer

Proc., 1976 Conf. Prec. Electromagn. Meas., 28 June-1 July 1976, Boulder, CO, pp. 382-385

June 1976 
ALLRED, C. M.; MANNEY, C. H.

The Calibration and Use of Directional Couplers Without Standards

IEEE Trans. Instrum. Meas., Vol. IM-25, No. 1, pp. 84-89 March 1976

HUDSON, P. A.

The National Measurement System for Medical Ultrasonics NBSIR 75-937

February 1976

ADAIR, R. T.

A Precision $30 \mathrm{MHz}$ Waveguide-Below-Cutoff Attenuator with an Absolute Electronic Readout, NBS Model XII

NBSIR 76-833

January 1976

ENGEN, G. F.

Automated Calibration of Directional-Coupler-Bolometer-Mount Assemblies

IEEE Trans. Microwave Theory Tech., Vol. MTT-23, No. 12, pp. 984-990

December 1975

HOER, C. A.; ROE, K. C.

Using an Arbitrary Six-Port Junction to Measure Complex Voltage Ratios IEEE Trans. Microwave Theory Tech., Vol. MTT-23, No. 12, pp. $978-984$

December 1975

HOER, C. A.

Using Six-Port and Eight-Port Junctions to Measure Active and Passive Circuit Parameters

NBS Tech. Note 673

September 1975

RANDA, M.; MAY, W. G.

A Millimeter Wave Reflection Beam Isolator

IEEE Trans. Microwave Theory Tech., Vol. MTT-23, No. 6, pp. 506-508

June 1975

ENGEN, G. F.

Automated Calibration of Directional-Coupler-Bolometer-Mount Assemblies

Proc., 1975 IEEE Microwave Theory and Technique Symp. Int.,

12-14 May 1975, Palo Alto, CA, pp. 98-99

May 1975

JESCH, R. L.; HOER, C. A.

Characterization of a High Frequency Probe Assembly for Integrated

Circuit Measurements

NBS Tech. Note 663

January 1975

ENGEN, G. F.

Calibration Technique for Automated Network Analyzers with Application to Adapter Evaluation

IEEE Trans. Microwave Theory Tech. Part II, 1974 Symp. Issue, Vol. MTT-22, pp. 1255-1260

December 1974 
ROMARER, E. I.; TRYON, P. V.

An Application of the Power Equation Concept and Automation Techniques to Precision Bolometer Unit Calibration

IEEE Trans. Microwave Theory Tech. Part II, 1974 Symp. Issue, Vol. MTT-22, pp. 1260-1267 December 1974

ENGEN, G. F.

An Alternative Calibration Technique for Automated Network Analyzers with Application to Adapter Evaluation Proc., 1974 IEEE S-MTT Intl. Microwave Symp., 12-14 June 1974, Atlanta, GA, pp. 261-262 June 1974

HUDSON, P. A.; MELQUIST, D. G.; ONDREJKA, A. R.; WERNER, P. E. Completion of the Program to Evaluate/Improve Instrumentation and Test Methods for Electroexplosive Device Safety Qualification NBSIR 74-379 June 1974

JONES, R. N.

The Measurement of Lumped Parameter Impedance NBS MN 141 June 1974

ROMARER, E. I.

An Application of the Power Equation Concept and Automation Techniques to Precision Bolometer Unit Calibration Proc., 1974 IEEE S-MTT Intl. Microwave Symp., 12-14 June 1974, Atlanta, GA, pp. 263-265 June 1974

SIMPSON, P. A.

Broadband Pulsed/CW Calibration Signal standard for Field Intensity Meter (FIM) Receivers NBSIR 74-371 June 1974

ENGEN, G. F.

Comments on Practical Analysis of Reflectometers and Power Equation Concepts IEEE Trans. Instrum. Meas., Vol. IM-23, pp. 104-105 March 1974

IARSON, W.; CAMPBELL, E.

Microwave Attenuation Measurement system (Series substitution)

NBS Tech. Note 647 February 1974

ENGEN, G. F. Calibration of an Arbitrary Six-Port Junction for Measurement of Active and Passive circuit Parameters IEEE Trans. Instrum. Meas., Vol. IM-22, pp. 295-299 December 1973 
WEIDMAN, M. P.; ENGEN, G. F.

Application of a Non-Ideal Sliding Short to Two-Port Loss Measurement NBS Tech. Note 644 october 1973

YATES, B. C.; COUNAS, G. J.

Summary of WR15 Flange Evaluation at $60 \mathrm{GHz}$ NBS Tech. Note 642 October 1973

ENGEN, G. F.

Mismatch Considerations in Evaluating Amplifier Noise Performance

IEEE Trans. Instrum. Meas., Vol. IM-22, pp. 274-278 September 1973

ALLRED, C. M.; MANNEY, C. H. Self-Calibration of Complex Ratio Measuring System

Proc., 1973 Intl. Conf. Meas, and Instrum., 17-23 June 1973, Dresden, Germany, Acta Imeko 1, pp. 157-166 June 1973

HOER, C. A.; ENGEN, G. F. Analysis of a Six-Port Junction for Measuring, V, I, A, B, Z, Gamma, and Phase

Proc., 1973 Intl. Conf. Meas. and Instrum., 17-23 June 1973, Dresden, Germany, Acta Imeko, Vol. 1, pp. 213-222 June 1973

ENGEN, G. F.

Theory of UHF and Microwave Measurements Using the Power Equation Concept

NBS Tech. Note 637

April 1973

NAHMAN, N. S.

A Note on the Transition (Rise) Time Versus Line Length in Coaxial Cables

IEEE Trans. Circuit Theory, Vol. CT-20, pp. 165-167 March 1973

ENGEN, G. F.; HOER, C. A.

Application of an 'Arbitrary' 6-Port Junction to Power Measurement Problems

IEEE Trans. Instrum. Meas., Vol. IM-21, Pp. 470-474 November 1972

HOER, C. A.

The Six-Port Coupler: A New Approach to Measuring Voltage, Current, Power, Impedance, and Phase

IEEE Trans. Instrum. Meas., Vol. IM-21, PP. 466-470 November 1972

FOOTE, W. J.; BUNTER, R. D.

Improved Gearing for Rotary-Vane Attenuators

Rev. Scientific Instrum., vol. 43, No. 7, pp. 1042-1043

July 1972 
YATES, B. C.; LARSON, W.

Millimeter Attenuation and Reflection Coefficient Measurement system NBS Tech. Note 619 July 1972

ENGEN, G. F.; BOER, C. A.

Application of an 'Arbitrary' 6-Port Junction to Power Measurement Problems

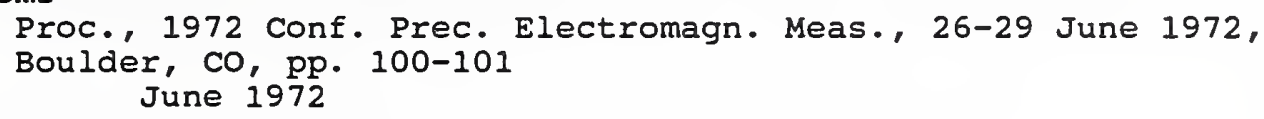

ESTIN, A. J.

A Calculable Microwave Attenuation Standard: The High Q Cavity

Proc., 1972 Conf. Prec. Electromagn. Meas.,

26-29 June 1972, Boulder, CO, pp. 100-101

June 1972

BOER, C. A.

The Six-Port Coupler; A New Approach to Measuring V, I, P, Z, and Theta

Dig., 1972 Conf. Prec. Electromagn. Meas.,

26-29 June 1972, Boulder, CO, pp. 6-29

June 1972

HUDSON, P. A.; SAULSBERY, L. F.

An Adjustable-slot-Length UHF Coaxial Coupler with Decade Bandwidth

IEEE Trans. Microwave Theory Tech., Vol. MTT-19, pp. 781-783 september 1971

ENGEN, G. F.; HUDSON, P. A.

International Intercomparison of Power Standards at $3 \mathrm{GHz}$

IEEE Trans. Microwave Theory Tech., Vol. MTT-19, pp. 411-413 April 1971

COOK, C. C.; AILRED, C. M.

An Excitation System for Piston Attenuators

IEEE Trans. Instrum. Meas., Vol. IM-20, pp. 10-16 February 1971

ENGEN, G. F.

Power Equations: A New Concept in the Description and Evaluation of Microwave Systems

IEEE Trans. Instrum. Meas., Vol. IM-20, pp. 49-57 February 1971 
ENGEN, G. F. (cont.)

Theorem Giving Limits for $S_{2}$ when $S_{11}$ and the Two-Port Efficiency are known

IEEE Trans. Instrum. Meas., Vol. IM-20, p. 78

February 1971

An Extension to the sliding short Method of Connector and Adaptor Evaluation NBS JRES, Vol. 75C, pp. 177-183 1971

A New Method of Characterizing Amplifier Noise Performance IEEE Trans. Instrum. Meas., Vol. IM-19, pp. 344-349 November 1970

HOER, C. A.; AGY, D. L.

Broad-Band Resistive-Divider-Type Directional Coupler

IEEE Trans. Instrum. Meas., Vol. IM-19, pp. 336-343 November 1970

CRAWFORD, M. L.; SMART, G. R.

New Coaxial Thermistor Mounts for Use as Precision Transfer Standards Proc., 1970 Instrument Soc. of America Conf., 26-29 October 1970, Philadelphia, PA, N708-70, pp. 1-6 October 1970

SIMPSON, P. A.; ONDREJKA, A. R.

Improvements to the NBS RF Peak-Pulse Power standard

1970 Instrument Soc. of America Silver Jubilee Intl. Conf. and Exhibit, October 1970, Philadelphia, PA, Paper 709-70, pp. 1-7

October 1970

WEIDMAN, M. P.; CAMPBELL, E.

A Method for Designing Multi-Screw Waveguide Tuners NBS Tech. Note 393 October 1970

AGY, D. L.; NELSON, R. E.

An Immittance Transcomparator

1970 Annual Precision Measurement Association Metrology Conf. (3rd), 17-18 June 1970, Gaithersburg, MD, Vol. 1, pp. 147-154
June 1970

scoTT, w. W., Jr.

A New Coaxial RF-DC Ammeter Proc., 1970 Conf. Prec. Electromagn. Meas., 2-5 June 1970, Boulder, Co, Spec. Issue Conf. Prec. Electromagn. Meas. Digest p. 13 June 1970

JONES, R. N . ; HUNTLEY, L. E. A Precision, High Frequency Calibration Facility for Coaxial Capacitance Standards

NBS Tech. Note 386

March 1970 
LAWTON, R. A.; AILRED, C. M. ; HUDSON, P. A.

A Wide Range CW Power Measurement Technique

IEEE Trans. Instrum. Meas., Vol. IM-19, pp. 28-34 February 1970

ENGEN, G. F. An Introduction to the Description and Evaluation of Microwave Systems Using Terminal Invariant Parameters NBS MN 112

october 1969

BEATTY, R. W.

Impedance Measurements and Standards for Uniconductor Waveguide Proc., IEEE Spec. Issue on Radio Meas. Methods and standards, Vol. 55, No. 6, pp. 933-941 June 1967

ELLERBRUCH, D. A.

UHF and Microwave Phase-shift Measurements Proc., IEEE Spec. Issue on Radio Meas. Methods and Standards, Vol. 55 , No. 6, pp. 960-969 June 1967

HUDSON, P. A.

Measurement of RF Peak Pulse Power

Proc., IEEE Spec. Issue on Radio Meas. Methods and Standards, Vol. 55 , No. 6, pp. $851-855$ June 1967

HUNTLEY, L. E.; JONES, R. N.

Lumped Parameter Impedance Measurements

Proc., IEEE Spec. Issue on Radio Meas. Methods and standards, Vol. 55 , No. 6, pp. 900-911 June 1967

JESCE, R. I.

Impedance Measurements in Coaxial Waveguide Systems Proc., IEEE Spec. Issue on Radio Meas. Methods and standards, Vol. 55, No. 6, pp. 912-923 June 1967

KERNS, D. M.

Definitions of $v, i, z, Y, a, b, \Gamma$, and $s$

Proc., IEEE Spec. Issue on Radio Meas. Methods and standards, Vol. 55 , No. 6, pp. 892-900 June 1967

ONDREJKA, A. R.

Peak Pulse Voltage Measurement (Baseband Pulse)

Proc., IEEE Spec. Issue on Radio Meas. Methods and Standards, Vol. 55 , No. 6, pp. 882-885 June 1967

RUMFELT, A. Y . : ELWELI, L. B.

Radio Frequency Power Measurements Proc., IEEE Spec. Issue on Radio Meas. Methods and standards, Vol. 55 , No. 6, pp. 837-850 June 1967 
RUSSELI, D. H.; LARSON, W.

RF Attenuation

Proc., IEEE Spec. Issue on Radio Meas. Methods and Standards, Vol. 55, No. 6, pp. 942-959

June 1967

SCOTT, W. W., Jr.; FREDERICK, N. V.

The Measurement of Current at Radio Frequencies Proc., IEEE Spec. Issue on Radio Meas. Methods and standards, Vol. 55, No. 6, pp. 886-891 June 1967

SELBY, M. C.

Voltage Measurement at High and Microwave Frequencies in Coaxial systems Proc., IEEE Spec. Issue on Radio Meas. Methods and standards, Vol. 55, No. 6, pp. 877-882 June 1967

RUSSELL, D. H.

An Unmodulated Twin-Channel Microwave Attenuation Measurement System Instrum. Soc. Amer. Conf. Vol. 4, pp. 162-169 April 1965 
ENGEN, G. F.; WAIT, D. F.

A "Terminal Invariant" Description of Amplifier Noise

Digest, 1992 Conf. Prec. Electromagn. Meas., 9-12 June 1992, Paris, France, pp. 250-251 June 1992

PERERA, $\mathbf{S}$.

"Broadband Mismatch Error" in Noise Measurement Systems

Digest, 1992 Conf. Prec. Electromagn. Meas., 9-12 June 1992,

Paris, France, pp. 256-257 June 1992

WAIT, D. F.

A Comparison of Three Techniqies for the Precision Measurement of Amplifier Noise

Digest, 1992 Conf. Prec. Electromagn. Meas., 9-12 June 1992,

Paris, France, pp. 252-253 June 1992

Measuring Accuracies for Various Techniques for Measuring Amplifier Noise

Digest, 39th Automatic RF Techniques Group Conf., 5 June 1992,

Albuquerque, NM, pp. 43-52 June 1992

DAYWITT, W. C.

Exact Principal Mode Field for a Lossy Coaxial Iine

IEEE Trans. Microwave Theory Tech., Vol. MTT-39, No. 8, pp. 1313-1322

August 1991

WAIT, D. F.; ENGEN, G. F.

Application of Radiometry to the Accurate Measurement of Amplifier Noise

IEEE Trans. Instrum. Meas., Vol. IM-40, No. 2, pp. 433-437 April 1991

WAIT, D. F.; COUNAS, G. J.; RESSEL, W.; BUCHHOLZ, F. IM.

PTB - NIST Bilateral Comparison of Microwave Noise Power in Coaxial Transmission Iine

IEEE Trans. Instrum. Meas., Vol. IM-40, No. 2, pp. 449-454 April 1991

DAYWITT, W. C.

First Order Symmetric Modes for a slightly Lossy Coaxial Transmission Line

IEEE Trans. Microwave Theory Tech., Vol. MTT-38, No. 11.

pp. 1644-1650

November 1990

WAIT, D. F.

Comments Concerning on-Wafer Noise Parameter Measurements

Digest, 36th Auto. RF Tech. Group Conf., 29-30 November 1990,

Monterey, CA, pp. 5-15

November 1990

DAYWITI, W. C.

First Order Principal Mode Fields and Distributed Line Parameters for a Slightly Lossy Coaxial Transmission Line.

Dig., Conf. Prec. Electromagn. Meas., 11-14 June 1990, Ottawa, Canada, pp. 404-405

June 1990 
PERERA, S.

A Simple Noise Calibration Radiometer

Dig., Conf. Prec. Electromagn. Meas., 11-14 June 1990, Ottawa, Canada, pp. 32-33 June 1990

WAIT, D. F : COUNAS, G. J.; RESSEL, W.; BUCHHOLZ, F. IM.

PTB - NIST Bilateral Comparison of Microwave Noise Power in Coaxial Transmission Line

Dig., Conf. Prec. Electromagn. Meas., 11-14 June 1990; Ottawa, Canada, pp. 34-35

June 1990

DAYWITT, W. C.: COUNAS, G. J .

Measuring Adapter Efficiency Using a sliding short Circuit IEEE Trans. Microwave Theory Tech., Vol. MTT-38, No. 3, pp. 231-237

March 1990

DAYWITI, W. C.

Radiometer Equation and Analysis of systematic Errors for the NIST

Automated Radiometers

NIST Tech. Note 1327

March 1989

GEYER, R. G.

Magnetostatic Measurements for Mine Detection

NISTIR 88-3098

October 1988

WAIT, D. F.; DAYWITT, W. C.

Intercomparison of NBS Noise Calibration Services

Dig., Conf. Prec. Electromagn. Meas., 7-10 June 1988, Tsukuba

Science City, Japan, pp. 209-210 1988

DAYWITT, W. C.

A Simple Technique for Determining Joint Losses on a Coaxial Iine from

Swept-Frequency Reflection Data

IEEE Trans. Instrum. Meas., Vol. IM-36, pp. 468-473 June 1987

Complex Admittance of a Lossy Coaxial open circuit with a Hollow Center Conductor

Intl. J. Sci. Metrol., Metrologia, Vol. 24, pp. 13-22 1987

A Simple Technique for Investigating Defects in Coaxial Connectors IEEE Trans. Microwave Theory Tech., Vol. MTT-35,

No. 4, pp. 460-464 April 1987

A Simple Technique for Determining Joint Losses on a Coaxial Iine from Swept-Frequency Reflection Data

Dig., 1986 Conf. Prec. Electromagn. Meas., 23-27 June 1986, NBS, Gaithersburg, MD, p. 40 June 1986

10-60 GHz G/T Measurements Using the Sun as a Source--A Preliminary Study

NBSIR 86-3046

April 1986 
WAIT, D. F.

The Impact of Automation on NBS Noise Temperature Measurements

Proc., IEEE Spec. Issue Radio Meas. Methods Standards, pp. 117-120 1986

HALFORD, D.

Transparent Metrology of Signal to Noise Ratios of Noisy Band-limited

Digital Signals

NBS Tech. Note 1077

June 1985

PERERA, $\mathbf{S}$.

Noise Temperature Measurements at the National Bureau of standards

Proc., 1985 Instrum. and Meas. Technol. Conf.,

20-22 March, 1985, Tampa, FL, pp. 159-160

March 1985

DAYWITT, W. C.

A Derivation for the Noise Temperature of a Horn-type Noise Standard Metrologia, 21 pp. 127-133 1985

Broadband Noise Source Applications

Proc., 1985 Instrum. and Meas. Technol. Conf.,

20-22 March, 1985, Tampa, FL, pp. 165-166 1985

An Error Analysis for the Use of Presently Available Lunar Radio Flux Data in Broadbeam Antenna-System Measurements

Proc., 1984 Antenna Applications Symp., 19-21 September 1984, University of Illinois, Urbana-Champaign, IL, pp. 1-26 september 1984

A Preliminary Investigation into Using the Sun as a Source for G/T Measurements

NBSIR 84-3015

August 1984

MIILER, C. R. S.; DAYWITT, W. C.

The NBS Switching Radiometers

NBSIR 84-3004

May 1984

The Reference Noise Standards NBS WR62 and WR90

NBSIR 84-3005

May 1984

DAYWITT, w. C.

A Coaxial Noise standard for the $1 \mathrm{GHz}$ to $12.4 \mathrm{GHz}$ Frequency Range NBS Tech. Note 1074

March 1984

An Error Analysis for the Use of Presently Available Lunar Radio Flux Data in Broadbeam Antenna-system Measurements

NBS Tech. Note 1073

February 1984

COUNAS, G. J.

NBS $2.0 \mathrm{GHz}$ to $4.0 \mathrm{GHz}$ Automated Radiometer Operation and Service Manual NBSIR 84-1697

January 1984 
DAYWITT, W. C.

Design and Error Analysis for the WR10 Thermal Noise Standard

NBS Tech. Note 1071

December 1983

WAIT, D. F.

Precision Measurement of Antenna System Noise Using Radio Stars

IEEE Trans. Instrum. Meas., Vol. IM-32, No. 1, pp. 110-116 March 1983

Earth Terminal Measurement System Operations Manual (Revised) NBSIR 83-1679(R) January 1983

WAIT, D. F.; DAYWITT, W. C.

Preliminary Examination of $20 \mathrm{GHz} \mathrm{G} / \mathrm{T}$ Measurements of Earth Terminals NBSIR 83-1686

March 1983

WAIT, D. F.

Precision Measurement of Antenna System Noise Using Radio Stars

Dig., 1982 Conf. Prec. Electromagn. Meas.,

28 June-1 July 1982 , Boulder, CO, p. F-17

June 1982

COUNAS, G. J.; BREMER, T. H.

NBS 30/60 Megahertz Noise Measurement System Operation and Service

Manual

NBSIR 81-1656

December 1981

WAREFIELD, J . P.

Addendum to Earth Terminal Measurement System Maintenance Manual

NBSIR 81-1641

October 1981

ESTIN, A. J.; DAYWITT, W. C.

Evaluation of Signal Plus Noise Detection Error in an Envelope Detector with Logarithmic Compression

IEEE Trans. Inf. Theory, Vol. IT-17, No. 5, pp. 663-664 September 1981

NBSIR 79-1614(R)

August 1979

DAYWITT, W. C.

An Error Analysis for the Measurement of Satellite EIRP using a Calibrated Radio star

IEEE Trans. Instrum. Meas., Vol. IM-27, No. 3, pp. 253-258 September 1978

Atmospheric Propagation Equations Used in the NBS Earth Terminal Measurement System

NBSIR 78-883

April 1978

WAIT, D. F.

Earth Terminal Measurement System Operations Manual

NBSIR 78-879

April 1978 
ESTIN, A. J.; DAYWITT, W. C.

Evaluation of Signal Plus Noise Detection Error in an Envelope Detector with Logarithmic Compression

Proc., 1978 Conf. Prec. Electromagn. Meas., 26-29 June 1978,

Ottawa, Canada 1978

DAYWITT, W. C.

Error Equations Used in the NBS Earth-Terminal Measurement System NBSIR 78-869

December 1977

A Precision Earth-Terminal System for Accurate C/KT, G/T, and EIRP Measurements with a Calibrated Radio Star

Proc., 1977 Union Radio Scientifique Internationale Intl. Symp. on Meas. in Telecommunications,

3-7 October 1977, Lannion, France, pp. 1-4

october 1977

RANDA, M.

An Improved Solid state Noise Source

IEEE Trans. Microwave Theory Tech. pp. 173-182 December 1976

DAYWITT, W. C.

Error Equations Used in the NBS Precision G/T Measurement System NBSIR 76-842

September 1976

RANDA, M.

Study of Errors in Absolute Flux Density Measurements of Cassiopeia A

IEEE Trans. Instrum. Meas., Vol. IM-25, No. 3, pp. 173-182

September 1976

An Improved Solid state Noise Source

Proc., 1976 IEEE MTT-S Microwave Symp., 14-16 June, 1976,

Cherry Hill, NJ, Pp. 224-226

June 1976

RANDA, M.; LARSON, T. R.

Stability of Silicon Avalanche Noise Diodes at $K$ and $K a$ Bands

Proc., 1976 Conf. on Precision Electromagn. Meas.,

28 June-1 July 1976, Boulder, CO

RANDA, $M$. June 1976

Study of Errors in Absolute Flux Density Measurements of Cassiopeia A NBSIR 75-822 October 1975

A Measure for the Stability of Solid State Noise Sources Proc., 1975 IEEE Intl. Microwave Theory and Technique symp., 12-14 May 1975, Palo Alto, CA, pp. 315-317 May 1975

DAYWITT, W. C.; KANDA, M.

G/T Measurement Errors with Radio Stars Proc., 1975 IEEE Intl. Antennas and Propagat. Symp., 2-4 June 1975, Urbana-Champaign, IL, Session 20, Pp. 460-463 June 1975

WAIT, D. F.; KANDA, M.; DAYWITT, W. C.; MILIER, C. R. S.

A Study of the Measurement of G/T Using Cassiopeia A NBSIR 74-382

October 1974 
ARTHUR, M. G.

The Measurement of Noise Performance Factors: A Metrology Guide NBS MN 142 June 1974

DAYWITT, W. C.

A Reference Noise Standard for Millimeter Waves IEEE Trans. Microwave Theory Tech., Vol. MTT-21, No. 12, pp. 845-847 December 1973

ENGEN, G. F. Mismatch Considerations in Evaluating Amplifier Noise Performance IEEE Trans. Instrum. Meas., Vol. IM-22, pp. 274-278 September 1973

HALFORD, D. Comparing Frequencies

Physics Today, Vol. 26, No. 2, p. 15 February 1973

BOYLE, D. R.; CLAGUE, F. R.; REEVE, G. R.; WAIT, D. F.; RANDA, M. An Automated Precision Noise Figure Measurement System at $60 \mathrm{GHz}$

IEEE Trans. Instrum. Meas., Vol. IM-21, No. 4, pp. 543-549 November 1972

BALFORD, D. Infrared-Microwave Frequency Synthesis Design: Some Relevant Conceptual Noise Aspects

Proc., 1971 Frequency Standards and Metrology Seminar, 30 August - 1 September 1972, Quebec, Canada, pp. 431-466 August 1972

DAYWITT, W. C.; FOOTE, W. J.; CAMPBELL, E. WR15 Thermal Noise standard

NBS Tech. Note 615 March 1972

ARTHUR, M. G.

A Precision HF-Noise Power Measurement System

Proc., Instrum. Soc. Amer. ISA-70 Conf., October 1971 October 1971

ENGEN, G. F.

A New Method of Characterizing Amplifier Noise Performance

IEEE Trans. Instrum. Meas., Vol. IM-19, pp. 344-349 November 1970 
GANS, W. L.; GEYER, R. G.; RLEMPERER, W. K.

Quantifying Standard Performance of Electromagnetic-Based Mine Detectors NISTIR 3982 October 1991

HILL, D. A.

Magnetic Dipole Excitation of an Insulated Conductor of Finite Length IEEE Trans Geosci. Remote Sensing, Vol. GE-28, No. 3, pp. 289-294 May 1990

HILI, D. A.; ANDERSON, L. R.

Propagation Along a Two-Wire Line Located at the Air-Earth Interface.

IEEE Trans. Geosci. Remote Sensing, Vol.GE-28, No. 3, pp. 400-402. May 1990

HILI, D. A.

Fields of Horizontal Currents Located Above the Earth

IEEE Trans. Geosci. Remote Sensing, Vol. GE-26, No. 6, pp. 726-732 November 1988

Magnetic Dipole Excitation of a Long Conductor in a Lossy Medium

IEEE Trans. Geosci. Remote Sensing, Vol. GE-26, No. 6, pp. 720-725 November 1988

Electromagnetic Wave Propagation in a symmetrical coal seam

IEEE Trans. Antennas Propagat., Vol. AP-34, No. 2, pp. 244-247 February 1986

Radio Propagation in a Coal Seam and the Inverse Problem Proc., 1985 Intl. Symp. on Antennas and EM Theory,

26-28 August 1985, Beijing, China, pp. 422-427 August 1985

BELSHER, D. R.; MCLAUGHIN, R. G.; REPJAR, A. G.; BUSSEY, H. E. Detection of Lost Wells and Unknown Water-filled Voids in Coal Mines through Development of an Experimental Microwave system NBSIR 84-3017

September 1984

HILL, D. A.

Radio Propagation in a Coal Seam and the Inverse Problem

NBS JRES, Vol. 89, No. 5, pp. 385-394

September-october 1984

CHEW, В.

Electromagnetic Modeling of oil shale Retorts for Remote Sensing Purposes

IEEE Trans. Geosci. Remote Sensing, Vol. GE-20, No. 4, pp. 510-517

October 1982

ROE, K. C.; WITTMANN, R. C.

Improved Coal Interface Detector

NBSIR 82-1663

May 1982

CHEW, H.

Modeling of Oil Shale Retorts for Electromagnetic sensing Techniques NBSIR 81-1653

November 1981 
BELSHER, D. R.

Feasibility study on the Use of a Microwave system for the

Nondestructive Evaluation of Historic Adobe structures

NBSIR 79-1610

July 1979

MONTGOMERY, J. P.; CHANG, D. C.

Electromagnetic Boundary-Value Problems Based Upon a Modification of

Residue calculus and Function Theoretic Techniques NBS MN 164

June 1979

BOYNE, H. S ; ELLERBRUCH, D. A.

Microwave Measurements of Snow Stratigraphy and Water Equivalence Proc., Western Snow Conference

April 1979

ELLERBRUCH, D. A.; JOHNSON, R. B.; BELSHER, D. R.; YAGHJIAN, A. D.;

STEPPE, M. C.; FLEMING R. W.

High Resolution Sensing Techniques for slope stability studies

Federal Highway Administration Report, FHWA-7-3-0001 March 1979

CHANG, A. T. C.; SHIUE, J. C.; BOYNE, H. S.; ELLERBRUCH, D. A.; COUNAS, G. J.

Preliminary Results of Passive Microwave Snow Experiment During February and March 1978

NASA-TP-1408, Rept-7802-F22, p. 114 1979

ELLERBRUCH, D. A.; BELSHER, D. R.

Electromagnetic Technique of Measuring Coal Layer Thickness

IEEE Trans. Geosci. Electron., Vol. GE-16, No. 2, pp. 126-133 April 1978

ELLERBRUCH, D. A.; JESCH, R. L.; JONES, R. N •; BUSSEY, H. E • ; BOYNE, H. S.

Electromagnetic Scattering Properties of Soils and Snow

Proc., 1978 Intl. Symp. on Remote sensing of Environment (12th),

20-26 April 1978, Ann Arbor, MI, Vol. 2, pp. 957-974

April 1978

BEREUTER, W. A.; CHANG, D. C.

Electromagnetic Remote Sensing of Inhomogeneous Media

NBSIR 76-851

January 1977

ELLERBRUCH, D. A. ; BELSHER, D. R.

FM-CW Electromagnetic Technique of Measuring Coal Layer Thickness NBSIR 76-840

May 1976

ELLERBRUCH, D. A.; ADAMS, J.W.

Microwave Measurement of coal Layer Thickness

NBSIR 74-387

September 1974

ELLERBRUCH, D. A.

Electromagnetic Attenuation Properties of clay and Gravel Soils NBSIR 74-381

August 1974 
STOLTENBERG, R. E.

Detection of Human Intruders by Low Frequency Sonic Interferometric Techniques NBSIR 74-364

May 1974

COLLIER, R. S.; ELLERBRUCH, D. A.

RF (Radiofrequency) Total Mass Gauging in Large storage Containers: Empty Tank Modes

NBSIR 73-346

October 1973

COLIIER, R. S.; ELIERBRUCH, D. A.; CRUZ, J. E.; STORES, R. W.;

LUFT, $P$. E .

Mass Quantity Gauging by RF Mode Analysis

NBSIR 73-318

June 1973

CRUZ, J. E.; ROGERS, E. H.; HIESTER, A. E.

Continuous Liquid Level Measurements with Time-Domain Reflectometry Adv. Cryo. Eng., Vol. 18, pp. 323-327 (Paper H-4) 1973 
RANDA, M.; ONDREJRA, A. R.

An Ultra-Broadband and Nondispersive Sensor for the Measurement of TimeDomain Signals

Proc., Symp. on Electromagnetic Security for Information

Protection, 21-22 November, 1991, Rome, Italy, pp. 65-78 November 1991

TOFANI, S.; ONDREJKA, A. R.; RANDA, M.

A Time-Domain Method for Characterizing the Reflection Coefficient of Absorbing Materials from 30 to $1000 \mathrm{MHz}$

IEEE Trans. Electromagn. Compat., Vol. 33, No. 3, pp. 234-240 August 1991

GANS, W. L.; GEYER, R. G.; KLEMPERER, W. R.

Suggested Methods and standards for Testing and Verification of Electromagnetic Buried Object Detectors NISTIR 88-3915

March 1990

ADAMS, J. W.; ONDREJKA, A. R.; CAVCEY, R. H.; CRUZ, J. E.; MEDLEY, H. W.; GROSVENOR, $\mathrm{J}$. B .

Recent Improvements in Time-Domain EMC Measurement system NISTIR 89-3927

November 1989

GEYER, R. G.

Magnetostatic Measurements for Mine Detection

NISTIR 88-3098

October 1988

ADAMS, J. W.; ONDREJRA, A. R.; MEDLEY, H. W.

Time-Domain System for Indentification of the Natural Resonant

Frequencies of Aircraft Relevant to Electromagnetic Compatibility

Testing

NBSIR $87-3077$

November 1987

LAWTON, R. A.; RIAD, S. M.; ANDREWS, J.R.

Pulse and Time Domain Measurements

Proc., IEEE, Special Issue on Radio Meas. Methods and Stds, Vol. 74, No. 1, pp. 77-81

January 1986

LAWTON, R. A.; ONDREJKA, A. R.

Standards for the Measurement of Impulsive Fields Radiated by a TEM Horn Antenna

Proc., 1977 Union Radio Scientifique Intl. E Comm.,

3-7 October 1977, Lannion, France, pp. 1-4 1977

ANDREWS, $\mathbf{J}$. R.

Impulse Generator Spectrum Amplitude Measurement Techniques

IEEE Trans. Instrum. Meas., Vol. IM-25, No. 4, pp. 380-384

December 1976

ANDREWS, J. R.; GANS, W. I.

Time Domain Automatic Network Analyzer

I'Onde Electrique, Vol. 55 No. 10, pp. 569-574

December 1975 
ANDREWS, J. R.; BALDWIN, E. E.

Baseband Impulse Generator Useful to $5 \mathrm{GHz}$ Proc., 1975 IEEE Intl. Symp. on Electromagn. Compat., 7-9 october 1975, San Antonio, TX, pp. 1-4

october 1975

GANS, W. L.; ANDREWS, J. R.

Time Domain Automatic Network Analyzer for Measurement of RF and Microwave Components

NBS Tech. Note 672

September 1975

NAHMAN, N. S.; JICKLING, R. M.

Frequency Domain Measurement of Baseband Instrumentation NBSIR 73-330

July 1973

NAFMAN, N. S.; JICKLING, R. M.; HOLT, D. R.

Reference-Waveform Generation Using Debye Dielectric Dispersion NBSIR 73-304

December 1972 


\section{WAVEFORM METROLOGY}

CHESNUT, S. M.; PAULTER, N. G., Jr.

Automatic Waveform Analysis and Measurement System User Manual NISTIR 3978

December 1991

TOFANI, S.; ONDREJKA, A. R.; RANDA, M.

A Time-Domain Method for Characterizing the Reflection Coefficient of Absorbing Materials from 30 to $1000 \mathrm{MHz}$

IEEE Trans. Electromagn. Compat., Vol. 33, No. 3, pp. 234-240 August 1991

ONDREJKA, A. R.; RANDA, M.

A Time-Domain Method for Measuring the Reflection Coefficient of

Microwave Absorbers at Frequencies Below $1 \mathrm{GHz}$

Digest, 1991 Antennas and Propagation Society Symp., 24-28 June

1991, London, Ontario, Canada, Vol. 3, pp. 1656-1663 June 1991

PAULTER, N. G., Jr.; MAJUMDAR, A.

A New Triple Correlation Technique for Measuring Ultrashort Laser Pulses Rev. Sci. Instrum., Vol. 62, No. 3, pp. 567-578

March 1991

A New Proposed Triple Correlator for the Measurement of Ultrashort Laser Pulses

Opt. Comm., Vol. 81, No. 1,2, pp. 95-100

February 1991

GANS, W. I.

Dynamic Calibration of Oscilloscopes and Waveform Recorders Using Pulse standards

Record, Instrumentation and Measurement Technology (IMTC) Conf.,

13-15 February 1990, San Jose, CA, Pp. 246-250

February 1990

LAWTON, R. A.; MEYER, R.

Waveform Standards for Electro-optics: A Pulse Duration Comparison

IEEE Trans. Microwave Theory Tech., Vol. MTT-35, No. 4,

pp. $450-453$

April 1987

GANS, W. I.

Picosecond Pulse Waveform Error Analysis at NBS

Proc., IEEE Spec. Issue on Radio Meas. Methods and Standards,

pp. 86-90

$$
\text { January } 1986
$$

RIAD, S. M.

The Deconvolution Problem: An Overview

Proc., IEEE, Spec. Issue on Radio Meas. Methods and Standards, pp. 82-85

$$
\text { January } 1986
$$

NAHMAN, N. S.

Software Correction of Measured Pulse Data

NATO ASI Series on Fast Electrical and Optical Meas., Vol. 1, Series E, No. 108, pp. 351-417 1986 
GANS, W. L。

Picosecond Pulse Measurements at NBS

Proc., 1985 Instrum. and Meas. Technol. Conf., 20-22 March 1985, Tampa, FL, pp. 142-144 March 1985

LAWTON, R. A.

An Efficient Antialiasing Filter

IEEE Trans. Instrum. Meas., Vol. IM-34, No. 4, pp. 570-573 December 1985

Pulse Waveform Standards for Electro-Optics

Proc., 1985 Topical Meeting on Picosecond Electronics and Opto-Electronics, 13-15 March 1985, Lake Tahoe, NV, pp. 205-206 March 1985

Status Report Transient Waveform Recorder standard Proc., 1985 Instrum. and Meas. Technol. Conf., 20-22 March 1985, Tampa, FL, pp. 154-155 March 1985

LAWTON, R. A.; NAHMAN, N. S .; BIGELOW, J. M.

A Solid State Reference Waveform Standard

IEEE Trans. Instrum. Meas., Vol. IM-33, No. 3, pp. 201-205 September 1984

LAWTON, R. A.

An Efficient Antialiasing Filter

Dig., 1984 Conf. Prec. Electromagn. Meas., 20-24 August 1984, Delft, The Netherlands, pp. 104-105 August 1984

LAWTON, R. A.; NAHMAN, N. S .; BIGELOW, J. M.

A Solid State Reference Waveform Standard

Dig., Instrum. and Meas. Technol. Conf., 17-18 January 1984, Long Beach, CA, pp. 74-76 January 1984

ANDREWS, J.R.; BELL, B. A.; BALDWIN, E. E. Reference Flat Pulse Generator NBS Tech. Note 1067 October 1983

IAWTON, R. A.

Photoconductive Switches Used for Waveform Generation at the National Bureau of standards Proc., 1983 Picosecond Optoelectronics Conf., SPIE - The International Society for optical Engineering, 24-26 August 1983, San Diego, CA, Vol. 439, pp. 88-94 August 1983

ANDREWS, J. R.; BEIL, B. A.; NAHMAN, N. S.; BAIDWIN, E. E. Reference Waveform Flat Pulse Generator

IEEE Trans. Instrum. Meas., Vol. IM-32, No. 1, pp. 27-32 March 1983

GANS, W. I.

The Measurement and Deconvolution of Time Jitter in Equivalent-Time Waveform Samplers

IEEE Trans. Instrum. Meas., Vol. IM-32, No. 1, pp. 126-133

March 1983 
NAHMAN, N. S.

Picosecond Domain Waveform Measurements; Status and Future Directions

IEEE Trans. Instrum. Meas., Vol. IM-32, No. 1, pp. 117-124

March 1983

LAWTON, R. A.

Performance Standards for Waveform Recorders

IEEE Trans. Nucl. Sci., Vol. NS-30, No. 1, pp. 263-266

February 1983

ANDREWS, J.R.; NAHMAN, N. S.; BELI, B. A.

Status of Reference Waveform Standards Development at NBS

Proc., 1981 Waveform Recorder Seminar, 15 October 1981, Boulder, CO, NBS SP 634 , pp. $69-88$

June 1982

LAWTON, R. A.

Measurement Needs and Techniques for Evaluation/Calibration,

Proc., 1981 Waveform Recorder Seminar, 15 October 1981, Boulder, CO, NBS SP 634

June 1982

NAHMAN, N. S.

Some Generic Waveform Recorder Problems

Proc., 1981 Waveform Recorder Seminar, 15 October 1981, Boulder, CO, NBS SP 634, pp. 1-5 June 1982

ANDREWS, J. R.; BELL, B. A.; NAHMAN, N. S .; BALDWIN, E. E. Reference Waveform Flat Pulse Generator

Dig., Conf. Prec. Electromagn. Meas. June 1982 June 1982

IAWTON, R. A.

Precision Picosecond-Microsecond Electromagnetic Waveform Measurement at NBS

NBS SP 628, pp. 392-407

June 1982

RANDA, M.

Time Domain Sensors and Radiators

Natl. Radio Science Meeting, January 1982, University of

Colorado, Boulder, Co

January 1982

GANS, W. I.; NAHMAN, N. S.

Shielded Balanced and Coaxial Transmission Lines - Parametric Measurements and Instrumentation Relevant to Signal Waveform

Transmission in Digital Service

NBSIR 81-1042

June 1981

ANDREWS, J. R.; BALDWIN, E. E.

Amplitude Calibrator for Oscilloscopes

NBSIR 81-1646

April 1981

NAHMAN, N. S.

Error Criteria and the Use of Reference Waveforms

Electron. Test, Vol. 4, No. 2, pp. 72-76

February 1981 
ANDREWS, $\mathbf{J}$. R.

The Estimation of the Pulse Waveform in the Calibration of Impulse Generators

Dig., Intl. Union of Radio Sci. (Union Radio Scientifique

Internationale) Meeting, January 1981, Boulder, Co, p. 87 January 1981

Pulse Reference Waveform Standards Development at NBS

Proc., ATE Seminar/Exhib., January 1981, Pasadena, CA,

p. IV. 13 through IV. 19

January 1981

NAHMAN, N. S.

Error Criteria and the Use of Reference Waveforms

Proc. ATE Seminar/Exhib., January 1981, Pasadena, CA,

pp. IV.21 through IV.28

January 1981

NAHMAN, N. S.; GUIITAUME, M. E.

Deconvolution of Duration Limited Waveforms

Dig., Union Radio Scientifique Internationale, January 1981,

Boulder, Co, p. 86

January 1981

NAHMAN, $\mathbf{N}$. $\mathbf{S}$.

Picosecond Domain Waveform Measurements (Japanese translation)

Nikkei Electron., Nikkei-McGraw Hill

April 1979

YOUNG, M.; IAWTON, R. A.

Measurement of Pulsed-Laser Power

NBS Tech. Note 1010

February 1979

NAHMAN, N. S.

Picosecond-Domain Waveform Measurements

Proc., IEEE, Vol. 66, pp. 414-423

April 1978

ANDREWS, J.R.; NAHMAN, N. S.

The Measurement of Pulse Transition Duration

Proc., Union Radio Scientifique Internationale symp.,

3-7 October, 1977, Lannion, France, pp. 159-164 october 1977

IAWTON, R. A.; YOUNG, M.

Laser-Mode Beating Used for Detector Frequency-Response Measurements

App1. Opt., Vol. 16, pp. 2703-2705

October 1977

GANS, W. L.

Present Capabilities of the NBS Automatic Pulse Measurement System

IEEE Trans. Instrum. Meas., IM-25, pp. 384-388 December 1976

RIAD, S. M.; NAHMAN, N. S.

Application of the Homomorphic Deconvolution for the Separation of TDR Signals Occurring in Overlapping Time Windows

IEEE Trans. Instrum. Meas., IM-25, pp. 388-391

December 1976 
LAWTON, R. A.; ANDREWS, J. R. Electrical strobing of Photoconductor Cuts Sampling Oscilloscope's Risetime

Laser Focus, pp. 62-65 November 1976

ANDREWS, J. R.; LAWTON, R. A.

Electrically Strobed Optical Waveform Sampling Oscilloscope Rev. Sci. Instrum., Vol. 47, pp. 311-313 March 1976

ANDREWS, J . R.

Impulse Generator spectrum Amplitude Measurement Techniques Dig., Conf. Prec. Electromagn. Meas., pp. 23-24 1976

GANS, W. L.

Present Capabilities of the NBS Automatic Pulse Measurement System

Dig., Conf. Prec. Electromagn. Meas., pp. 25-26 1976

RIAD, S. M.; NAHMAN, N. S. Application of the Homomorphic Deconvolution for the separation of TDR Signals Occurring in Overlapping Time Windows

Dig., Conf. Prec. Electromagn. Meas. pp. 27-29 1976

ANDREWS, J . R.

Directional-Coupler Technique for Triggering a Tunnel Diode

IEEE Trans. Instrum. Meas., IM-24, pp. 275-277 september 1975

LAWTON, R. A.; ANDREWS, J. R. Pulsed-Laser Application to sampling oscilloscope Electron. Lett., Vol. 11, pp. 138 April 1975

ANDREWS, J. R.; GANS, W. L.

Pulsed Wavemeter Timing Reference for sampling Oscilloscope Calibration IEEE Trans. Instrum. Meas., IM-24, p. 82 March 1975

LAWTON, R. A.; SCAVENNEC, A. Photoconductive Detector of Fast-Transition Optical Waveforms Electron. Lett., Vol. 11, pp. 74-75 February 1975

ANDREWS, $\mathbf{J}, \mathbf{R}$.

Measurement on Pulses and Pulse Transmission Media, Circuits and Components

Intl. Union of Radio Sci. (Union Radio Scientifique

Internationale) Review of Radio Science, p. 9 $1975-1977$

Precision Picosecond-Pulse Measurements Using a High Quality Superconducting Delay Line

IEEE Trans. Instrum. Meas., IM-23, pp. 468-472

December 1974 
ANDREWS, J.R.; IAWTON, R. A.

Picosecond Pulse Research at NBS

Proc., Joint Meas. Conf., 12-14 November 1974, Gaithersburg, MD, pp. 123-140

November 1974

ANDREWS, J. R.

Precision Picosecond-Pulse Measurements Using a High Quality

Superconducting Delay Iine

Proc. Conf. Prec. Electromagn. Meas., 1-5 July 1974, London,

England, pp. 316-318

July 1974

SCAVENNEC, A.; NAHMAN, N. S.

Active and Passive Mode Locking of Continuously Operating Rhodamine 6G

Dye Lasers

NBSIR 73-347

February 1974

ANDREWS, J. $\mathbf{R}$.

Inexpensive Laser Diode Pulse Generator for Optical Waveguide Studies Rev. Sci. Instrum., Vol. 45, pp. 22-24

January 1974

HONDA, T.: NAHMAN, N. S.

Application of Ultrashort Optical Pulses to Electrical Pulse Measurements

Proc. Symp. on New Instrum., 28-29 January 1974, IEE of Japan, Hokkaido University, pp. 29-38 January 1974

ANDREWS, J. R.

Random Sampling Oscilloscope for the Observation of Mercury Switch

Closure Transition Times

IEEE Trans. Instrum. Meas., IM-22, pp. 375-381

December 1973

GANS, W. L.; NABMAN, N. S.

Pulse Testing of $R F$ and Microwave Components

NBSIR 1973

July 1973

ANDREWS, J . R.

Random Sampling Oscilloscope Time Base NBSIR 73-309

June 1973

HOLT, D. R.; NAHMAN, N. S.

Coaxial Line Pulse-Response Error Due to a Planar Skin Effect

Approximation

IEEE Trans. Instrum. Meas., IM-21, Pp. 515-519

November 1972

HONDA, T.; NABMAN, N. S.

A Dye Modelocked Nd+3 Glass Laser for Generating Electrical Reference Waveforms

NBSIR 74-360

September 1972 
NAHMAN, N. S.; HOLT, D. R.

Transient Analysis of Coaxial Cables Using the Skin Effect Approximation

$A+B$ (the square root of $s$ )

IEEE Trans. Circuit Theory, CT-19, pp. 443-451

september 1972

BOUILIE, R.; ANDREWS, J. R.

Measurement of Broadening of Pulses in Glass Fibers

Electron. Lett., Vol. 8, pp. 309-310

June 1972

HOLT, D. R.; NAHMAN, N. S .

Coaxial Line Pulse-Response Error Due to a Planar Skin Effect

Approximation

Proc., 1972 Conf. Prec. Electromagn. Meas., 26-29 June 1972,

Boulder, $\mathrm{CO}, \mathrm{pp} .1-2$

June 1972

IAWTON, R. A.; NAHMAN, N. S.

Pyroelectric Detector Application to Baseband Pulse Measurements Electron. Lett., Vol. 8, pp. 318-330

June 1972

ANDREWS, J. R.

A Frequency Calibrator for UHF Using an Avalanche Transistor

Q S T, Vol. 56, pp. 16-18

May 1972

ANDREWS, J.R.; WHITTEMORE, T.; MCCAA, W. D. Jr.

Survey of Present Waveform Sampling system Limitations

NBS Rept. 10731

February 1972

ANDREWS, J. R.

An Interfacing Unit for a 28 psec Feedthrough Sampling Oscilloscope

Rev. Sci. Instrum., Vol. 42, pp. 1882-1885

December 1971

Horizontal Time Base Sweep Generator for a Traveling Wave Oscilloscope

IEEE Trans. Nucl. Sci., NS-18, pp. 3-18

October 1971

EKSTROM, M. P.; MCCAA, W. D. Jr.; NAEMAN, N. S.

The Measured Time and Frequency Response of a Miniature superconducting Coaxial Line

IEEE Trans. Nucl. Sci., NS-18, pp. 18-25

October 1971

ANDREWS, J. R.; NAEMAN, N. S .

Electron-Beam Deflection in Traveling-Wave Oscilloscopes

Record of Symp. on Electron, Ion, and Laser Beam Technol. (11th), 12-14 May 1971, Boulder, Co

May 1971

MCCAA, W. D. Jr.; NAHMAN, N. S.

Generation of Reference Waveforms by Uniform Lossy Transmission Lines

IEEE Trans. Instrum. Meas., Vol. IM-19, pp. 382-390

November 1970 
ANDREWS, J. R.

Improved Bias Supply for Tunnel-Diode Picosecond Pulse Generators

IEEE Trans. Instrum. Meas., Vol. IM-19, pp. 171-175

August 1970

NAHMAN, N. S.; ERSTROM, M. P.; MCCAA, W. D. Jr.

Measured Time and Frequency Response of a Miniature Superconducting Coaxial Line

Applied Superconductivity Conf., June 1970, Boulder, Co June 1970 


\section{MISCELIANEOUS}

LYONS, R. M.; GIBSON, R. A.

A Bibliography of the NIST Electromagnetic Fields Division Publications NISTIR 3993

August 1992

A Bibliography of the NIST Electromagnetic Fields Division Publications NISTIR 3973

August 1991

A Bibliography of the NIST Electromagnetic Fields Division Publications NISTIR 3945

August 1990

FITZGERRELL, R. G.

Mobile Antennas

Natl. Inst. Justice Std.-0205.01

May 1989

LYONS, R. M.; GIBSON, K. A.

A Bibliography of the NIST Electromagnetic Fields Division Publications NISTIR 89-3920

September 1989

VANZURA, E. J.

Creating cSUBs in Basic, Part II

HP Design and Automation Magazine, p. 25

November 1988

Creating cSUBs in BASIC, Part I

HP Design and Automation Magazine, pp. 18-21

October 1988

REIDY, A. R.; GIBSON, R. A.

A Bibliography of NIST Electromagnetic Fields Division Publications NISTIR $88-3900$

September 1988

VANZURA, E. J.

creating cSUBs Written in FORTRAN That Run in BASIC

Proc., INTEREX Technical Computer Users Conf.,

7-12 August 1988, Orlando, FI, paper 20, pp. 1-18

August 1988

JESCH, R. I.

Fixed and Base station FM Receivers

Natl. Inst. Justice Std.-0206.01

July 1988

Fixed and Base station FM Transmitters

Natl. Inst. Justice Std. - 0201.01

September 1987

BENSEMA, W. D.

Mobile FM Transceivers

Natl. Inst. Justice Std. - 0210.00

May 1986

JESCH, R. L.

Personal/Mobile FM Transceivers

Natl. Inst. Justice Std. - 0224.00

May 1986 
GIBSON, R. A.; PAGE, J. M.; MILLER, C. K. S .

A Bibliography of the NBS Electromagnetic Fields Division Publications NBSIR 85-3040

February 1986

BENSEMA, W. D.

Personal FM Transceivers

Natl. Inst. Justice std.- 0209.01

September 1985

JESCH, R. I.

Measured Vehicular Antenna Performance

IEEE Trans. Veh. Technol., Vol. VT-34, No. 2, pp. 97-107 May 1985

GIBSON, R. A.; MILLER, C. K. S.

A Bibliography of the NBS Electromagnetic Fields Division Publications NBSIR 85-3022

April 1985

I.ARSEN, N. T.; VECCHIA, D. F.; SUGAR, G. R.

VOR Calibration Services

NBS Tech. Note 1069

April 1985

KUFFEL, J.; MALEWSKI, R.; VAN HEESWIJK, R.; IAWTON, R. A.

Dynamic Performance of Digital Recorders Used for Monitoring High

Voltage Impulse Tests

Proc., 1985 Instrum. and Meas. Technol. Conf., 20-22 March 1985,

Tampa, FL, pp. 211-215 March 1985

HIL工, D. A.

Book Review - Leaky Feeders and Subsurface Radio Communications

IEEE Antennas Propagat., Vol. IM-25, No. 3, pp. 23-24

June 1983

JESCE, R. I.

Dielectric Measurements of Oil Shale as Functions of Temperature and Frequency

NBSIR 83-1683

January 1983

HII工, D. A.

HF Ground Wave Propagation over Forested and Built-up Terrain

NTIA Report 82-114

December 1982

CHEW, H.

Electromagnetic Modeling of oil shale Retorts for Remote sensing

Purposes

IEEE Trans. Geosci. Remote Sensing, Vol. GE-20, No. 4, pp. 510-517

October 1982

GIBSON, R. A.; MILIER, C. K. S .

Bibliography of the Electromagnetic Fields Division Publications NBSIR 82-1673

August 1982

STOLTENBERG, R. E.; WITTMANN, R. C.

Acoustical Interferometer Wader AIW Final Report of Advanced Development

Test and Evaluation

NBSIR 82-167(R)

June 1982 
ROE, R. C.; WITTMANN, R. C. Improved Coal Interface Detector NBSIR 82-1663 May 1982

HIIL, D. A.; WAIT, J.R. Ground Wave Propagation over a Mixed Path with an Elevation Change IEEE Trans. Antennas Propagat., Vol. AP-30, No. 1, pp. 139-141 January 1982

NELSON, R. E.; JICKLING, R. M. Control Cable Assemblies Used with Mobile FM Receivers Natl. Inst. Justice std. - 0216.00 December 1981

CHEW, H. Modeling of Oil Shale Retorts for Electromagnetic Sensing Techniques NBSIR 81-1653 November 1981

YAGHJIAN, A. D.

Augmented Electric- and Magnetic-Field Integral Equations Radio science, Vol. 16, No. 6, pp. 987-1001 November-December 1981

HILU, D. A.; WAIT, J.R.

HF Ground Wave Propagation over Mixed Land, Sea, and Sea-Ice Paths IEEE Trans. Geosci. Remote Sensing, Vol. GE-19, No. 4, pp. 210-216

October 1981

HF Radio Wave Transmission over sea-Ice and Remote Sensing Possibilities IEEE Trans. Geosci. Remote Sensing, Vol. GE-19, No. 4, pp. 204-209

October 1981

WAREFIELD, J.P.

Addendum to Earth Terminal Measurement System Maintenance Manual NBSIR 81-1641

October 1981

CLARR, H. E.

Requirements for an Effective National Nonionizing Radiation Measurement system

NBS SP 613

June 1981

LEWIS, R. L.

Spherical Scanning Data Processing: An Algorithm for Halving the Data Processing Effort When the Radiation into the Back Hemisphere is Negligible

Dig., 1981 IEEE Ant. Propagat. Soc. Intl. Symp., June 1981 June 1981

YAGHJIAN, A. D. A Delta-Distribution Derivation of the Electric Field in the Source Region

Dig., 1981 IEEE Ant. Propagat. Soc. Intl. Symp., June 1981 June 1981 
HILL, D. A.; WAIT, J.R.

HF Ground Wave Propagation over Sea Ice for a Spherical Earth Model IEEE Trans. Antennas Propagat., Vol. AP-19, No. 3, pp. 525-527 May 1981

YAGHJIAN, A. D.

Reply to Criticism on Electric Dyadic Functions in the source Region Proc., IEEE, Vol. 69 , No. 2

February 1981

BOWMAN, R. R.

A Temperature Probe for Radio-Frequency Heated Material NBSIR 81-1634

January 1981

HIIL, D. A.; WAIT, J.R.

Electromagnetic Characteristics of a Coaxial Cable with Periodic slots IEEE Trans. Electromagn. Compat., Vol. EMC-22, No. 4, pp. 303-307

November 1980

MILLER, C. R. S.

A Bibliography of Publications in the NBS Electromagnetic Fields

Division

NBSIR 80-1635

November 1980

JONES, R. N.

Evaluation of Three-Terminal and Four-Terminal Pair Capacitors at High Frequencies

NBS Tech. Note 1024

September 1980

WAIT, J.R.; HILI, D. A.

Fields of a Horizontal Loop of Arbitrary Shape Buried in a Two-Layer Earth

Radio Science, Vol. 15, No. 5, pp. 903-912

September-October 1980

YAGHJIAN, A. D.

Augmented Electric- and Magnetic-Field Integral Equations

Dig., Union Radio scientifique Internationale Symp. on EM Waves, Munich Germany

August 1980

DRIVER, I. D.; RIES, F. X.

A Wideband RF Voltage Comparator

Dig., 1980 Conf. Precision Electromagn. Meas., 23-27 June 1980, Braunschweig, FDR, pp. 487-488 June 1980

HILL, D. A.; WAIT, J. R.

Ground Wave Attenuation Function for a Spherical Earth with Arbitrary Surface Impedance Radio science

Radio Science, Vol. 15, No. 3, pp. 637-643 May-June 1980

On the Excitation of the Zenneck Surface Wave Over the Ground at $10 \mathrm{MHz}$ Annales des Telecommunications, Vol. 35 , Nos. 5-6, pp. 1-4 May-June 1980 
HILL, D. A.; WAIT, J.R. (cont.)

Electromagnetic Theory of the Loosely Braided Coaxial Cable: Part II Numerical Results

IEEE Trans. Microwave Theory Technol., Vol. MTT-28, No. 4, pp. 326-331

April 1980

Propagation Along a Coaxial Cable with a Helical Shield

IEEE Trans. Microwave Theory Technol., Vol. MTT-28, No. 2, pp. 84-89

February 1980

WYSS, J. C.; STWALLEY, W. C.; ROCH, M. E.; VERMA, R. R.

Laser Fluorescence Diagnostics of Lithium Vapor for Inertial Confinement Applications

Topical Meeting on Inertial Confinement Fusion, February 1980, San Diego, CA February 1980

YAGHJIAN, A. D.

Electric Dyadic Green's Functions in the Source Region

Proc., IEEE, Vol. 68 , No. 2

February 1980

ELLERBRUCH, D. A.; BOYNE, H. S .

Active Microwave Water Equivalence Measurements Proc., Ft. Collins snow Workshop 1980

HAMILTON, C.; ILOYD, F.; PETERSON, R.; ANDREWS, J.R.

A Superconducting Sampler for Josephson Logic Circuits

Appl. Phys. Lett., Vol. 35, No. 9, pp. 718-719 November 1979

WHARTON, M. J.; FRUSH, C. L.; NAHMAN, N. S .

A Video Recorder for Coherent Doppler Radar IEEE Trans. Geosci. Electron., Special Issue on Radio Metrology, $\mathrm{GE}-17$, No. 4, pp. 171-178 October 1979

ARTHUR, M. G.; REEVE, G. R. Modulation Factor Standards NBS Tech. Note 1016 September 1979

NATMAN, N. S.; ALIRED, C. M.; ANDREWS, J.R.; HOER, C. A.; LAWTON, R. A. Radar Absorber Measurement Techniques at Frequencies Above $20 \mathrm{GHz}$ NBSIR 79-1613

August 1979

MONTGOMERY, J. P.; CHANG, D. C. Electromagnetic Boundary-Value Problems Based Upon a Modification of Residue Calculus and Function Theoretic Techniques NBS MN 164

June 1979

JONES, R. N.

A Comparison of Centrifuge and Freezing Calorimeter Methods for Measuring Free Water in snow NBSIR 79-1604

April 1979 
RING, J.; PEISER, H. S.; SANGSTER, R. C.

Guidelines for specification and Procurement of Measurement

Instrumentation NBSIR 78-891

June 1978

SANGSTER, R. C.

Collected Executive Summaries: Studies of the National Measurement

system

NBSIR 75-947-Rev. S.

February 1978

YAGHJIAN, A. D.

A Direct Approach to the Derivation of Electric Dyadic Green's Functions NBS Tech. Note 1000

January 1978

JESCH, R. L.

Evaluation of Low-Loss/Low-Reflection Two-Port Devices or Adapters by

Automated Measurement Techniques NBSIR 78-870

December 1977

JICKLING, R. M.; SHAFER, J. F.

FM Repeater systems

Natl. Inst. Justice Std. - 0213.00

November 1977

YOUNG, M.; ILAWTON, R. A.

Saturation of Optical Detectors at High Modulation Frequency

Proc., Annual Meeting opt. Soc. of America, 12 October 1977

Toronto, Canada, Vol. 67, p. 1398

October 1977

ZAPF, T. L.; HARVEY, M. E.; LARSEN, N. T.; STOLTENBERG, R. E.

Ultrasonic Calorimeter for Beam Power Measurements from 1 to

15 Megahertz

Proc., 1976 Ultrasonics Symp., 29 September-1 October 1977,

Annapolis, MD, IEEE Cat 76, CH1120-5SU, pp. 573-576

September 1977

NELSON, R. E.

A Guide to Voice Scramblers for Law Enforcement Agencies

NBS SP 480-8

December 1976

SANGSTER, R. C.

Study of the National Measurement System. Final Summary Report, 1972-75 NBSIR 75-925

December 1976

YAGHJIAN, A. D.

A Direct Approach to the Derivation of Electric Dyadic Green's Functions

Dig., AP Symp., October 1976, pp. 76-156

October 1976

ZAPF, T. L.; HARVEY, M. E.; LARSEN, N. T.; STOLTENBERG, R. E.

Ultrasonic Calorimeter for Beam Power Measurements from 1 to

15 Megahertz

NBS Tech. Note 686

September 1976 
SANGSTER, R. C.

Collected Executive Summaries: Studies of the National Measurement System 1972-75 NBSIR 75-947

September 1976

Transactions Matrix Description of the National system of Physical Measurements NBSIR 75-943

August 1976

LAWTON, R. A.; ANDREWS, J. R. Optically Strobed Sampling Oscilloscope

IEEE Trans. Instrum. Meas., Vol. IM-25, pp. 56-60 March 1976

RANDA, M.; MAY, W. G.

Reflection Beam Isolator for Submillimeter Wavelength NASA-CR-140761 June 1975

KERNS, D. M. Scattering-Matrix Description and Near-Field Measurements of Electroacoustic Transducers

J. Acoust. Soc. Amer., Vol. 57, No. 2, pp. 497-507 February 1975

JESCH, R. L.; HOER, C. A.

Characterization of a High Frequency Probe Assembly for Integrated Circuit Measurements

NBS Tech. Note 663

January 1975

ROGERS, G. J.; SAWYER, D. E .; JESCE, R. I. Semiconductor Measurement Technology: Measurement of Transistor scattering Parameters NBS SP 400-5 January 1975

KANDA, M.; MAY, W. G. Hollow-Cylinder Waveguide Isolators for use at Millimeter Wavelengths NASA-CR-140760 November 1974

JICRLING, R. M.; SHAFER, J. F.

Repeaters for Law Enforcement Communication Systems-Law Enforcement Standard Program Natl. Inst. Justice std. - 0206.00 october 1974

LITTLE, W. E.

Automated Computer Controlled Measurements

Proc., 1974 Conf. Preci. Electromagn. Meas., 1-5 July 1974, London, England July 1974

BUSSEY, H. E.; LARSEN, E. B. Buried Antenna Performance: Development of Small Resonant Buried Antennas

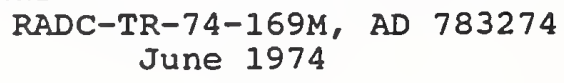


JESCE, R. I.; BAILEY, R. A.; TAUSCH, H. J .

Characterization of A H.F. Probe for Integrated Circuits Measurements Proc., Government Micro-Circuit Applications Conf., 25-27 June 1974, Boulder, CO, pp. 120-121 June 1974

WEITZEL, D. H.; LOWE, L. T.; ELLERBRUCH, D. A.; CRUZ, J. E.; SINDT, C. F. Hydrogen Slush Density Reference System NASA-CR-124764 June 1974

YAGHJIAN, A. D. Direct Integration of the Field Equations for Electroacoustic Transducers

IEEE Lett., Vol. 62, No. 6, pp. 858-859 June 1974

STOLTENBERG, R. E. Detection of Human Intruders by Low Frequency Sonic Interferometric Techniques NBSIR 74-364 May 1974

MILLER, C. K. S.

Millimeter Wave Metrology Capabilities at NBS Proc., 1974 Millimeter Waves Techniques Conf., 26-28 March 1974, San Diego, CA, p. 11 March 1974

YAGHJIAN, A. D.

Theory of Adjoint Reciprocity for Electroacoustic Transducers NBSIR 73-329

February 1974

SCAVENNEC, A.; NABMAN, N. S .

A Simple Passively Mode-Locked CW Dye Laser

IEEE J. Quantum Electron., Vol. QE-10, pp. 95-96 January 1974

WAIT, D. F . BEATTY, R. W.

The 1973 Intl. Microwave symp.

IEEE Trans. Microwave Theory Technol., Vol. MTT-21, No. 12, pp. 747-751

December 1973

ELIERBRUCH, D. A.

Test Results for the Mooring Line Data Line

NBSIR 73-341

October 1973

HALFORD, D.; SHOAF, J. H.; RISLEY, A. S . Spectral Density Analysis: Frequency Domain Specification and Measurement of Signal stability 1973 Annual Symp. on Frequency Control (27th), 12-14 June 1973, Cherry Hill, NJ, pp. 421-431 June 1973

SCOTT, W. W., Jr. Batteries Used with Law Enforcement Communications Equipment: Chargers and Charging Techniques NBS Rept. 10732 June 1973 
STOLTENBERG, R. E.

RF Null Detector NBS/SND

NBSIR 73-302

June 1973

BERRY, L. A.; FITZGERREIL, R. G.; VOGLER, I. E.

Investigation of Effect of Antenna Site and Antenna Type on LF

Non-Directional Beacon Performance FAA-RD-73-174

1973

ELLERBRUCH, D. A.

Application of Measurements of Electromagnetic Phenomena to Oceanography Proc., 1972 UJNR/MEC Symp. (lst), Record of the U.S.-Japan UJNR Joint Symp. on Marine Electronics, (Japanese Electrical Industrial Committee, Tokyo, Japan), 9 October 1972, Tokyo, Japan, pp. 23-30 october 1972

HALFORD, D.

Infrared-Microwave Frequency Synthesis Design: Some Relevant Conceptual Noise Aspects

Proc., 1971 Frequency Standards and Metrology Seminar, 30 August-1 September 1971, Quebec, Canada, pp. 431-466

August 1972

SANGSTER, R. C.

Relevancy of Measurements by a Systems Approach

Proc., 1972 Joint Meas. Conf., 21-23 June 1972, Boulder, Co, pp. $31-37$

June 1972

MANNEY, C. H.

Applicability of Speech Bandwidth Compression Techniques in Mine

Electromagnetic Communications

NBS Rept. 10728

February 1972

JESCH, R. I.; BERRY, I. S.

Batteries Used with Law Enforcement Communications Equipment

NBS Rept. 10722

October 1971

KANDA, $\mathbf{M}$.

Non-Reciprocal Devices Using Solid state Magnetoplasmas at Millimeter

and Submillimeter Wavelengths

NASA-CR- 122923

August 1971

COLLIER, R. S.; CRUZ, J. E.; ELLERBRUCH, D. A.; LOWE, I. T.; WEITZEL, D. H. Instrumentation for Hydrogen Slush storage Containers

NASA-CR-119241

June 1971

NBS Rept. 9793

June 1971

WEITZEL, D. H.; LOWE, L. T.; ELLERBRUCH, D. A.; CRUZ, J. E.; SINDT, C. F. Hydrogen Slush Density Reference System

NBS Rept. 9796

June 1971 
BRANDENBERGER, H.; HADORN, F.; HALFORD, D.; SHOAF, J. H.

High Quality Quartz Crystal Oscillators: Frequency Domain and Time Domain Stability

Proc., 1971 Frequency Control Annual Symp. (25th),

26-28 April 1971, Atlantic City, NJ, pp. 226-234

April 1971

TAGGART, H. E.; WORKMAN, J. L.; NELSON, R. E.

Emergency Locator Transmitters: Effective Radiated Power Levels and Techniques of Determining Effective Radiated Power NBS Rept. 9769

February 1971

ELIERBRUCH, D. A.

Microwave Methods for Cryogenic Liquid and Slush Instrumentation

IEEE Trans. Instrum. Meas., Vol. IM-19, No. 4, pp. 412-416 November 1970

scotT, W. W., Jr.

New Coaxial RF-DC Ammeter

IEEE Trans. Instrum. Meas., Vol. IM-19, No. 4, pp. 318-323 November 1970

LEWIS, R. L.

The Wave-Hop Fields for an Inclined Dipole Over a Spherical Earth with

an Anisotropic Ionosphere OT-ITR-RR5

October 1970

WEIDMAN, M. P.; CAMPBELI, E.

A Method for Designing Multi-Screw Waveguide Tuners

NBS Tech. Note 393

october 1970

FOOTE, W. J.

A New Flange Design for o-Ring seals

Rev. Sci. Instrum.

August 1970

HAYES, R. E.; RANDA, M.; MASCLET, G.; MAY, W. G.

The Application of Semiconductors to Quasi-optical Isolators for use at Submilimeter Wavelengths

NASA-CR-86379

April 1970

WACKER, P. F.; CORD, M. S.; BURKHARD, D. G.; PETERSEN, J. D.; KUKOL, R. F. Microwave Spectral Tables, Volume III, Polyatomic Molecules with Internal Rotation NBS MN 70, Vol 3 . June 1969

BOWMAN, R. $R$. Self-Modulating Transducers EOS-7039-Final 1968 
ADAIR, R. T. . . . . . . . . . . . . . . . . . . 63-68, 74 ADAMS, J. W. $.27,28,30,32,33,35,37,39,40,43,44,47,52,53,55-58$,

AGEE, D. A.

. . . . . 88,90

$A G Y, D . L$.

ALIEN, J. W.

ALLRED, C. $M$.

ALSPACH, W. J.

ANDERSON, L. R.

ANDREWS, J. R.

ANSON, W. J.

ARTHUR, M. G.

$6 \dot{6}, 7 \dot{3}, 7 \dot{4}, 7 \dot{6}, 7 \dot{7}, 7 \dot{0}, 104$

AULD, B. A.

BABJI, T. D.

$66,73,74,76,77,79,104$

87

BAILEY, R. A.

BAIRD, R. C.

BAKER-JARVIS, J.R.

BALDWIN, E. E.

BEAN, J . L .

BELL, B. A. -

BELSHER, D. R.

BENNETT, $W . S$.

BENSEMA, $W$. D.

BEREUTER, $W$. A.

BERRY, I. S.

BERRY, I. A.

BIGELOW, J. M.

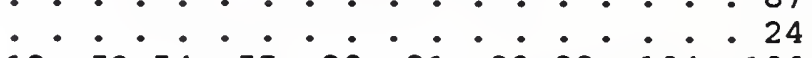

$19,52-54,57,90,91,93-99,104,106$

BLACKBURN, D.

$4 \dot{7}, 5 \dot{1}, 5 \dot{3}, 5 \dot{7}, 69,83,33,43$

BORSERO, $M$.

BOUILLE, $R$.

.. . . . . . . 22

13,46

BOYLE, D. R.

BOYNE, H. S.

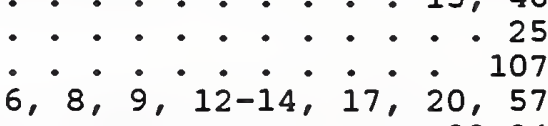

BRANDENBERGER, H

$22-24$
93,94

BREMER, T. $\mathrm{H}$.

BUCHHOLZ, F. IM

BUDLONG, A.

BURKHARD, D. G

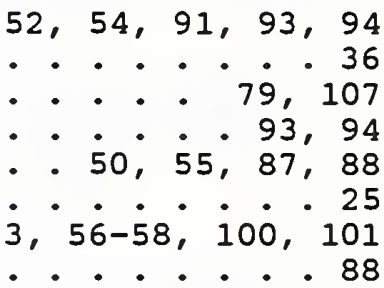

BURNS, J. G.

BUSSEY, H. E.

CAMELL, D. G

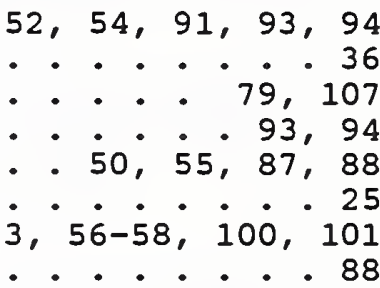

CAMPBELI, E.

$1 \dot{2}, 14,3 \dot{1}, 3 \dot{2}, 3 \dot{5}, 3 \dot{6}, 4 \dot{2}, 4 \dot{5}, 4 \dot{8}, 5 \dot{3}, 5 \dot{6}-\dot{58}, 10 \dot{0}, 101$

CAPOBIANCO, Т. Е. . . . . . . . . . . . . . . . . . . . . . . 22

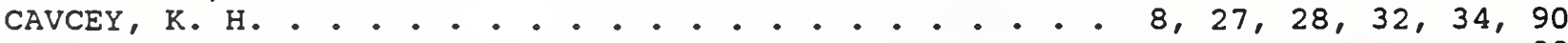

CHANG, A. T. C. . . . . . . . . . . . . . . . . . . . . . . . 88 CHANG, D. C. . . . . . 12, 13, 31, 33-35, 41, 45, 47-52, 54, 55, 88, 104

CHESNUT, S. M.

CHEW, H. - .

CLARK, A. V.

$49, \dot{54}, \dot{55}, \dot{57}, \dot{58,} \dot{103,} 109$

CLARK, H. E.

108
108

COLIIER, R. S

COOK, C. C.

- $87, i 0 i, 102$

$59-61,63,65,86$

CORD, M. S.

48,102

89,108

COUNAS, G. J.

CRAWFORD, $M$. L.

CRUZ, J. E.

DAVIDSON, A.

DAY, G. W.

$63,76,81-84,88$

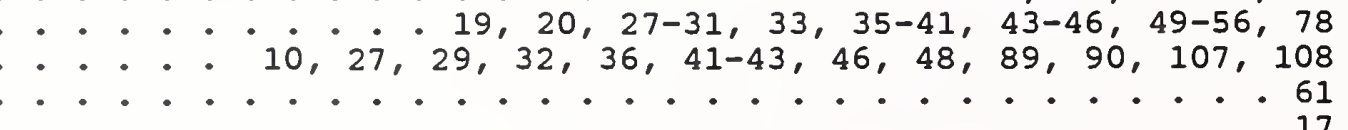

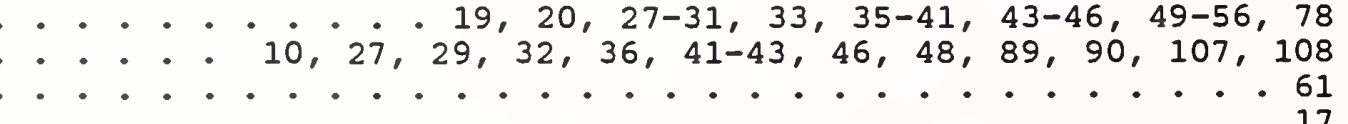

DAY, G. W.

\begin{abstract}
-
\end{abstract}


DAYWITT, W. C.

$8,11,12,16-19,24,81-86$

DECKER, W. F.

- 51

DOBROSKI， H.

22,23

DOMICH, P. D.

$\cdot 16$

DOUGHERTY, H. T.

$27,28,31-33,36-38,41,44,46,47,70,103$

EBBESEN, H.

. 69

EHRET, R. L.

98,99

EKSTROM, M. P.

ELLERBRUCH, D. A.

$25,79,88,89,104,107-109$

ELWELI, L. B. $64-66,68-79,81,86$

ENGEN, G. F.

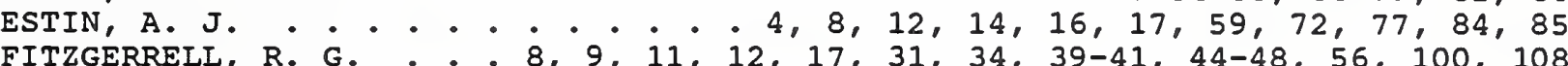

FLANIGAN, W. F. Jr. . . . . . . . . . . . . . . . . . . . 54

FLEMING R. W.

$76,86,109$

FOOTE, W. J.

FRANCIS, M. H.

$3-11,13$

FREDERICK, N. V.

FREE, G. M.

FRIDAY, D. S.

60,77

FRUSH, C. L.

FURLOW, R.

GALLIANO, P. G.

GANS, W. L.

GATTERER, L. E.

GEYER, R. G.

GIBSON, K. A.

GINLEY, R. A.

GREENE， F. M.

GREENLEE, D. H.

GRIMM, K. R.

GROSVENOR, J. H.

GUERRIERI， J. R.

GUILLAUME, M. E.

GUPTA, $K$. C.

HADORN, F.

HALFORD, D.

HAMILTON, C.

HARVEY, M. E.

HAYES, R. E.

$32,34,39,40$

. . . 60

$13,40,46$

90-97

... 64,66

$22-24,82,87,90$

- 100,101

.. .66

$54,56,57$

- 12,13

$22,23,90$

HIESTER, A. E. . .

HILI, D. A. $5,7-10,12,15,24,27-30,32,34-36,38-42,44,49,50,69,87$,

HOER, C. A. .............. 63,64,66-74,76-78, 104, 106

HOLT, D. R. . . . . . . . . . . . . . 62-64, 67, 91, 97, 98

HONDA, T. . . . . . . . . . . . . . . . . . . . . . . . . 97

HUANG, D. X. . . . . . . . . . . . . . . . . . . . . 59

HUDSON, P. A. . . . . . . ...... 56, 58, 69,73-75, 77, 79

HUNTER, R. D. . . . . . . . . . . . . . . . . . . 76

HUNTLEY, L. E. . . . . . . . . . . . . . . . . . . 78,79

JANEZIC, M. D. . . . . . . . . . . . . . . . . . . . . 4, 22-24

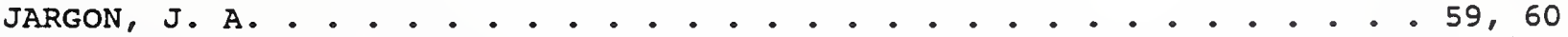

JESCH, R. L. $9,10,24,25,33,35,37,39,67,71,72,74,79,88,100,101$,

$91,102,105,106$

JOHNK, R. T. . . . . . . . . . . . 27,28

JOHNSON, R. B. . . . . . . . . . . . . . . . . . . . 88 JONES, R. N. . . . . . . . 25, 60,68,69, 75, 78, 79, 88, 103, 104 JUDISH, R. M. . . . . . . . . . . . . . 61,64,66,68 JUROSHEK, J.R. . . . . . . . . . . . . . . 60,63,65-68 KAISER, R. K. F. . . . . . . . . . . . . . . . . . . 63 KAMPER, R. A. . . . . . . . . . . . . . . . . . 68,70,72 KANDA, M. . . 8, 9, 11-13, 15-20, 27-38,40-47, 49-58, 74, 85, 86, 90, 92, 94,

KERNS, D. M. 
LADBURY, J. M.

LARSEN, E. B.

$9,10,19,20,28-33,36,37,440,42,43,48,49,53,54,57$,

LARSEN, N. T.

$64,65,71-73,101,105$

LARSON, T. R.

LARSON, W.

LAWTON, R. A.

LEWIS, R. L.

IITTLE, W. E.

IIU, B. H.

IIVINGSTON, $\dot{E} \cdot \mathrm{M}$.

LIOYD, $F$.

LOWE, I. T. .

LOWELL, $W$. R.

IUFT, P. E.

IYONS, R. M.

MA, M. T.

MACREYNOLDS, K.

MAJOR, J. R.

MAJUMDAR, A.

MALEWSKI， R.

MANNEY, C. H.

MARKS, R. B.

MARLER, F. E.

MARTZLOFF, F. D.

$\because \cdot \cdot 75,77,80$

MASCLET, G. -

MASTERSON, K. D.

MAY, W. G. • .

MCFARLANE, E. J.

MCLAUGHLIN, R. G.

MEDLEY, H. W. .

MELQUIST, D. G.

MET ZKER， R. F.

MEYER, K.

MIERS, T.

MILLER, C. K. S.

$16,2 \dot{4}, 2 \dot{5}, 3 \dot{9}, 5 \dot{2}, 5 \dot{8}, 7 \dot{9}, 9 \dot{0}, 9 \dot{2}-\dot{9}, \dot{101}, 104-106$

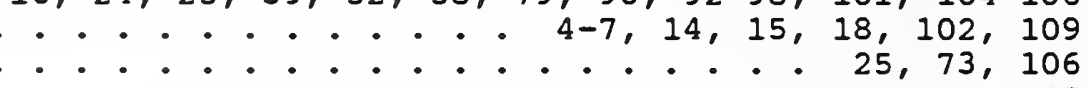

MONTGOMERY, J. P.

$6 \dot{3}, 6 \dot{6}-68$

- 104

107,108

MORGAN, J. M.

MORGAN, W. L.

MORRIS, D.

MUTH, I. A.

NAHMAN, N. S.

NELSON, R. E.

NEWELL, A. C.

NORGARD, J. D.

ONDREJKA, $\dot{A} . \dot{R}$.

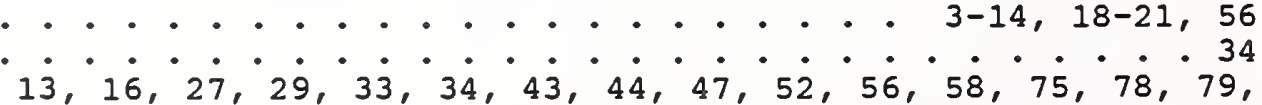

$O R R$, R. D. $8,9,11,27,28,30,31,33,37,41,43,46,47$

PAGE, J. M

PAULTER, N. G., Jr.

PEISER, H. S.

PERERA, $S$.

PERSINGER, R. R.

PETERSEN, J. D .

$12,13,15-17 ; 28-40,42-51$

PETERSON, R.

$63,66-68$

- $\quad 39,101$

$74,76,108$

. .63

PHILLIPS, K. R.

$44,4 \dot{6}, 4 \dot{7}, 4 \dot{9}, 5 \dot{2}, 6 \dot{7}, 8 \dot{3}, 8 \dot{5}, 10 \dot{0}, \dot{10}, 107$

88,104

$27,28,30-32,40$

$74,106,109$ 
WALKER, D. K. . . . . . . . . . . . . . . . . . . . . . . . 59

WEIDMAN, M. P. . . . . . . . . . . 63, 67, 69,71, 72, 76, 78, 109

WEIL, C. M. . . . • . . . . . . . . . . . . . . . . . $22,23,63$

WEITZEL, D. H. . . . . . . . . . . . . . . . . . . . . . 107, 108

WERNER, P. E. . . . . . . . . . . . . . . . . . . . . 56, 58, 75

WHARTON, M. J. . . . . . . . . . . . . . . . . . . . . . . . 104

WHITTEMORE, T. . . . . . . . . . . . . . . . . . . . . . . . . . . 98

WILLIAMS, D. F. . . . . . . . . . . . . . . . . . . . 22, 59-63

WILSON, P. F. . . . . . . . . . . 13, 29, 33, 34, 36, 38-45, 47, 49

WILSON, W. A. . . . . . . . . . . . . . . . . . . . . . . . . 51

WITTMANN, R. C. . . . . . . . . . . . . 3-7, 9, 11, 87, 101, 102

WOODRUFF, J. J. . . . . . . . . . . . . . . . . . . . . . . . . . 16

WORKMAN, J. L. . . . . . . . . . . . . . . . . . . . 14, 50-53, 109

WU, D. I. . . . . . . . . . . . . . . . . 27, 28, 30, 31, 33-35

WU, Y. . . . . . . . . . . . . . . . . . . . . . . . . . . . 53

WYSS, J. C. . . . . . . . . . . . 10, 11, 13, 37, 42, 43, 47, 104

YAGHJIAN, A. D. . . . . . . . . . . 3, 9, 12-15, 17-19, 88, 102-105, 107

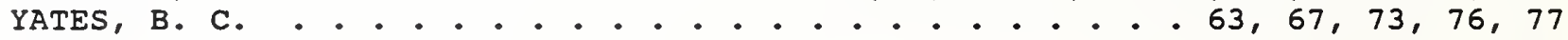

YODOSHIMA, I. . . . . . . . . . . . . . . . . . . . . . . . . . 40

YOUNG, M. . . . . . . . . . . . . . . . . . . . . . . . 95, 105

ZALTSMAN, E. B. . . . . . . . . . . . . . . . . . . . . . . . . 25

ZAPF, T. L. . . . . . . . . . . . . . . . . . . . . . . 73,105 
SYNC79-1 PRISM BLK

Entire record displayed.

STD: 03160

AUTH DI "GIESON, KATHRYN A"

Recond $t$ of i

AUTHORITY RECORD

AFN: 881592

ENTERED: 19830404

FEC STAT: NEW

VERIFIED

NAME/SUEJECT

REPLACED: 19846322

LC

ESTABLISHED HEADING

1010 ก 92227196

$2 \quad 640 \quad$ DGPO \& DLO

310010 Gibson, Kathryn A. [AACRZ]

4 G76 Her Bibliography of the NBS Electromagnetic Fialds Division

publications, 1982: b top. Kathryn A. Gibson, Electromagnetic Fields Div., Center for Electronics and Electrical Engingaring, Netional Engineering Lab. Boulder, Col 


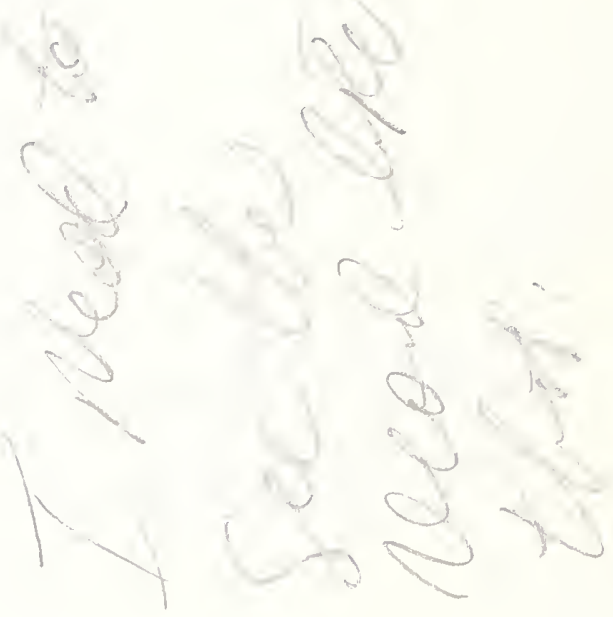




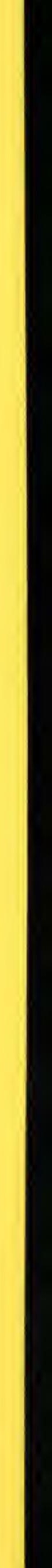

